\title{
Meso-free boron(III)subchlorin and its $\mu$-oxo dimer with interacting chromophores
}

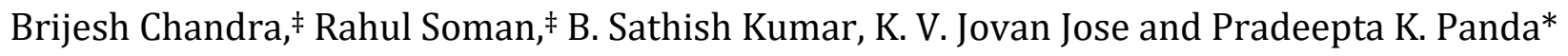

School of Chemistry, University of Hyderabad, Hyderabad, India-500046 


\section{Contents}

1. Instrumentation and Reagents

2. Synthesis

2. a. Synthesis of meso-diester-tripyrromethane

2. b. Synthesis of 1a (axial hydroxo meso-diester subchlorin)

2. c. Synthesis of $\mathbf{1 b} \& \mathbf{1 c}$ (axial alkoxo meso-diester subchlorin):

2. d. Synthesis of $\mathbf{2}$ (Subchlorin $\mu$-oxo dimer) from 1a (axial hydroxo subchlorin monomer)

2. e. Interconversion of subchlorin 1a to subporphyrin $\mathbf{3}$

3. NMR spectra of synthesised compounds (1-3)

4. HRMS data (1-3)

5. Single Crystal XRD analysis (1-3)

6. Absorption and emission studies

7. Fluorescence life time analysis

8. Singlet Oxygen Luminescence and Quantum yield analysis

9. Cyclic Voltammograms

10. Computational Analysis: Structure, Energetics, and Spectra

11. References 


\section{INSTRUMENTATION AND REAGENTS}

Nuclear magnetic resonance spectra were recorded on Bruker Avance- $400 \mathrm{MHz}$ and 500 MHz FT NMR spectrometer and Jeol A600 NMR spectrometer, tetramethylsilane (TMS, $\delta=0$ ) used as an internal standard at room temperature. The mass spectral determinations were carried out by Bruker Maxis HRMS and Waters Xevo G-2XS QTOF spectrometer by ESI techniques. Melting points were determined by a Lab India MR-VIS ${ }^{+}$visual melting point apparatus. All absorption spectra were recorded using Perkin Elmer Lambda-750 UV-VIS spectrometer.

The fluorescence and singlet oxygen emission spectra were recorded in Fluorolog-3-221 spectrofluorometer equipped with NIR detector. Fluorescence lifetime measurements were carried out using a time-correlated single-photon counting (TCSPC) spectrometer (Horiba Jobin Yvon IBH). PicoBrite diode laser source $\left(\lambda_{\text {exc }} 405 \mathrm{~nm}\right)$ was used as the excitation source and an MCP photomultiplier (Hamamatsu R3809U-50) as the detector. The pulse repetition rate of the laser source was $10 \mathrm{MHz}$. The width of the instrument response function, which was limited by the fwhm of the exciting pulse, was around $55 \mathrm{ps}$. The lamp profile was recorded by placing a scatterer (Ludox solution in water) in place of the sample. The time-resolved emission decay profiles were collected at steady-state emission spectrum maxima's $630 \mathrm{~nm}$ and $680 \mathrm{~nm}$. Decay curves were analyzed by nonlinear least-squares iteration procedure using IBH DAS6 (Version 2.2) decay analysis software. The quality of the fit was assessed by inspection of the $\chi^{2}$ values and the distribution of the residuals.

The steady-state luminescence of singlet oxygen of $\mathbf{1 , 2}$ and $\mathbf{3}$ were measured by using a Fluorolog-3-221 spectrofluorometer equipped with Hamamatsu H10330-75 TE cooled NIR detector. Free base meso-tetraphenylporphyrin $\left(\mathrm{H}_{2} \mathrm{TPP}\right)$ was taken as standard $\left(\Phi_{\Delta} 0.7\right)$. All samples showed the emission bands ranging from 1240 to $1320 \mathrm{~nm}$ with a broad peak around 1270 $\mathrm{nm}$. The singlet oxygen quantum yields $\Phi_{\Delta}$ for all samples can be determined by using eq $\mathrm{S} 1$ (comparative actinometry method): ${ }^{\mathrm{S} 1}$

$$
\Phi_{\Delta}=\Phi_{\Delta}^{s t d} \frac{I}{I_{s t d}} \frac{1-10^{-A^{s t d}}}{1-10^{-A}}
$$

where (0.7) is the singlet oxygen quantum yield of $\mathrm{H}_{2} \mathrm{TPP}$ as the standard sample in aerated toluene, $I$ and $I_{\text {std }}$ are the singlet oxygen emission intensities at the peaks for the tested sample and the reference standard ( $\left.\mathrm{H}_{2} \mathrm{TPP}\right)$, respectively; $\mathrm{A}$ and $\mathrm{A}_{\text {std }}$ refers to the ground-state absorbance of the tested sample and the reference standard $\left(\mathrm{H}_{2} \mathrm{TPP}\right)$ at the excited wavelength.

Cyclic voltammetric (CV) and differential pulse voltammetric (DPV) measurements were done using Zahner Zennium Electrochemical Workstation and electrodes were purchased from $\mathrm{CH}$ Instruments Inc. All measurements were done in dichloromethane under the flow of nitrogen, and $0.1 \mathrm{M}$ tetrabutylammoniumhexafluorophosphate $\left(\mathrm{TBAPF}_{6}\right)$ used as a supporting electrolyte. glassy carbon as a working electrode, platinum wire as counter electrode and $\mathrm{Ag} / \mathrm{AgCl}$ as a reference electrode were used. Ferrocenium/Ferrocene, $\mathrm{Fc}^{+} / \mathrm{Fc}$ couple was used as an external reference for calibration. The redox potentials were referenced vs. saturated calomel electrode (SCE). All cyclic voltammetric data were recorded at $50 \mathrm{mV} / \mathrm{s}$ scan rate. 
Crystallographic data for 1a, $\mathbf{2}, \mathbf{3}$ were collected on BRUKER APEX-II CCD microfocus diffractometer, Mo-K $\alpha(\lambda=0.71073 \AA)$ radiation and $\mathrm{Cu}-\mathrm{K} \alpha(\lambda=1.54184 \AA)$ radiation was used

to collect the $\mathrm{X}$-ray reflections of the crystal. Data reduction was performed using Bruker SAINT software. ${ }^{\text {S2 }}$ Intensities for absorption were corrected using and SADABS 2014/5, ${ }^{\text {S3 }}$ refined using SHELXL2014/7 ${ }^{\text {S4 }}$ with anisotropic displacement parameters for non-H atoms. Hydrogen atoms on $\mathrm{O}$ and $\mathrm{N}$ were experimentally located in difference electron density maps. All $\mathrm{C}-\mathrm{H}$ atoms were fixed geometrically using HFIX command in SHELX-TL. A check of the final CIF file using PLATON $^{\mathrm{S} 5}$ did not show any missed symmetry.

Crystallographic data (including the structure factor) for structures 1a, 2, 3 in this paper have been deposited in the Cambridge Crystallographic Data Centre as supplementary publication number CCDC 2023966, 2023967, 2023968, respectively. Copies of the data can be obtained free of charge on application to CCDC, 12 Union Road, Cambridge CB2 1EZ, UK (Fax: +44(0)-1223336033 or e-mail: deposit@ccdc.cam.ac.uk).

\section{SYNTHESIS \& CHARACTERISATION}

\section{2. a. Synthesis of meso-diester tripyromethane 6:}
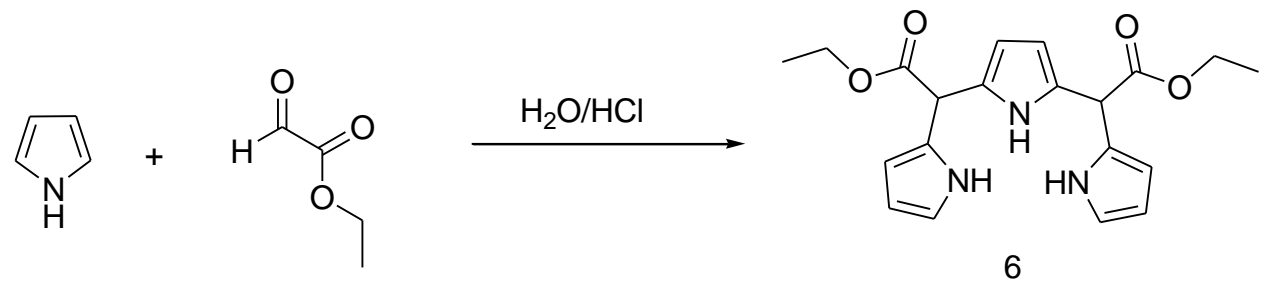

6

Pyrrole $(1.7 \mathrm{~mL}, 24.50 \mathrm{mmol})$ and $50 \%$ ethylglyoxalate in toluene $(2.4 \mathrm{~mL}, 12.10 \mathrm{mmol})$ were added in a $2 \mathrm{~L}$ round bottomed flask containing deionized water $(1.7 \mathrm{~L})$ and conc. $\mathrm{HCl}(35 \%)(10.0$ $\mathrm{mL})$. The reaction mixture was stirred at room temperature for $24 \mathrm{~h}$, followed by quenching with aqueous ammonia till the reaction mixture become neutral. The aqueous layer was extracted with dichloromethane, and the organic layer was washed with water and dried over anhydrous sodium sulfate. The solvent was removed in vacuuo. The crude product was loaded on silica gel column and TPM 6 was eluted with hexane/ethyl acetate (15\%) as an off-white pasty solid in its pure form.

Yield: $750 \mathrm{mg}(17 \%),{ }^{1} \mathrm{H}$ NMR $\left(400 \mathrm{MHz}, \mathrm{CDCl}_{3}\right) \delta(\mathrm{ppm}) 8.74$ (s, br, 1H, NH), 8.55 (s, br, 2H, $\mathrm{NH}), 6.73(\mathrm{~m}, 2 \mathrm{H}, \beta-\mathrm{CH}), 6.17(\mathrm{~m}, 2 \mathrm{H}, \beta-\mathrm{CH}), 6.08(\mathrm{~s}, 2 \mathrm{H}, \beta-\mathrm{CH}), 6.00(\mathrm{~m}, 2 \mathrm{H}, \alpha-\mathrm{CH}), 5.06(\mathrm{~s}$, $2 \mathrm{H}$, meso-H), $4.24\left(\mathrm{q}, \mathrm{J}=7 \mathrm{~Hz}, 4 \mathrm{H},-\mathrm{CH}_{2} \mathrm{CH}_{3}\right), 1.31\left(\mathrm{~m}, 6 \mathrm{H},-\mathrm{CH}_{2} \mathrm{CH}_{3}\right) .{ }^{13} \mathrm{C} \mathrm{NMR}(125 \mathrm{MHz}$, $\left.\mathrm{CDCl}_{3}\right) \delta(\mathrm{ppm}) 171.6,127.4,126.7,118.2,108.4,107.5,107.2,61.8,44.1,14.1$. HRMS (ESITOF) $\mathrm{m} / \mathrm{z}:[\mathrm{M}+\mathrm{Na}]^{+}$Calcd for $\mathrm{C}_{20} \mathrm{H}_{23} \mathrm{~N}_{3} \mathrm{O}_{4} \mathrm{Na} 392.1586$; Found 392.1588. 


\section{2. b. One-pot synthesis of $1 \mathrm{a}$ and 2 :}

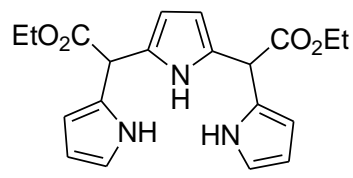

6

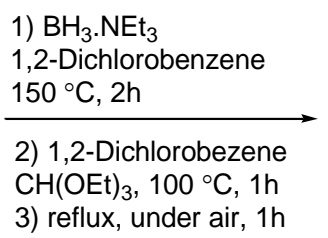

3) reflux, under air, $1 \mathrm{~h}$

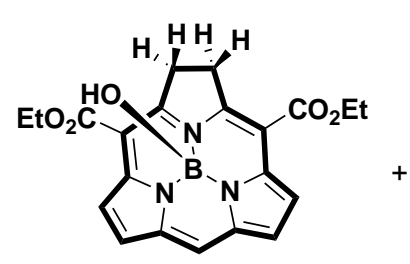

1 a $(12 \%)$

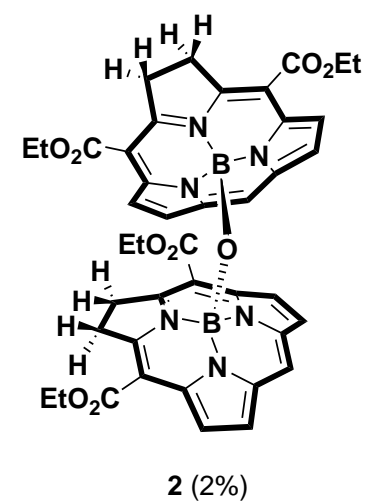

Freshly prepared diester tripyrrane $6(1.8 \mathrm{~g}, 5.01 \mathrm{mmol})$ dissolved in 1,2-dichlorobenzene $(50 \mathrm{~mL})$ and borane triethylamine complex $(2.22 \mathrm{~mL}, 15.03 \mathrm{mmol})$ was added to it and the reaction mixture was heated in an oil bath at $150{ }^{\circ} \mathrm{C}$ for $2 \mathrm{~h}$ under nitrogen atmosphere. The reaction mixture was allowed to cool to $100{ }^{\circ} \mathrm{C}$ and was slowly diluted with 1,2-dichlorobenzene (700 mL) containing triethylorthoformate $(20 \mathrm{~mL}, 120.24 \mathrm{mmol})$ was added and the reaction mixture was heated with gentle stirring at $100{ }^{\circ} \mathrm{C}$ for $1 \mathrm{~h}$ in a nitrogen atmosphere. After $1 \mathrm{~h}$, nitrogen atmosphere was removed and the reaction mixture was heated at reflux under open air atmosphere for $1 \mathrm{~h} .1,2-$ dichlorobenzene was removed in vacuum and the black residue was subjected to alumina filter column to remove polypyrrole impurity, the compound was further purified in neutral alumina column, using EtOAc/ Hexane (2:3) as eluent. Subchlorin $\mu$-oxo dimer fraction eluted as a red colour band, followed by the major B(III)subchlorin monomer as orange-red band with intense green fluorescence. The solvent was removed under reduced pressure to afford the subchlorin as dark red amorphous solid.

1a: Yield: $238 \mathrm{mg}(12 \%)$, m.p: $239.8-240.9{ }^{\circ} \mathrm{C} ;{ }^{1} \mathrm{H}$ NMR $\left(400 \mathrm{MHz}, \mathrm{CDCl}_{3}\right) \delta$ (ppm) 8.40 (s, $1 \mathrm{H}$, meso-H), $7.90(\mathrm{~d}, J=4.0 \mathrm{~Hz}, 2 \mathrm{H}, \beta-\mathrm{CH}), 7.79(\mathrm{~d}, J=4.4 \mathrm{~Hz}, 2 \mathrm{H}, \beta-\mathrm{CH}), 4.56(\mathrm{q}, J=7.2 \mathrm{~Hz}$, $\left.4 \mathrm{H}, \mathrm{CH}_{3} \mathrm{CH}_{2}-\right), 4.41(\mathrm{dd}, J=13.6 \mathrm{~Hz}$ and $20.4 \mathrm{~Hz}, 1 \mathrm{H}$, methylene), 4.38 (dd, $J=13.6 \mathrm{~Hz}$ and 20.4 $\mathrm{Hz}, 1 \mathrm{H}$, methylene), 4.01 (dd, $J=13.6 \mathrm{~Hz}$ and $20.0 \mathrm{~Hz}, 1 \mathrm{H}$, methylene), 4.39 (dd, $\mathrm{J}=13.6 \mathrm{~Hz}$ and $20.0 \mathrm{~Hz}, 1 \mathrm{H}$, methylene), 1.56 (t, J = 7.2 Hz, $\left.6 \mathrm{H}, \mathrm{CH}_{3} \mathrm{CH}_{2}-\right) ; 0.54$ (s, br, $1 \mathrm{H}$, axial $\mathrm{OH}$ ); ${ }^{11} \mathrm{~B}$ NMR $\left(128 \mathrm{MHz}, \mathrm{CDCl}_{3}\right)\left(\mathrm{BF}_{3} . \mathrm{OEt}_{2}\right.$ as internal standard) $\delta(\mathrm{ppm})-11.97 .{ }^{13} \mathrm{C} \mathrm{NMR}\left(100 \mathrm{MHz}, \mathrm{CDCl}_{3}\right)$ $\delta(\mathrm{ppm}) 166.8,158.8,144.3,134.7,123.6,117.5,114.9,102.3,60.9,33.6,14.6$. HRMS (ESI-MS) $\mathrm{m} / \mathrm{z}$ : $[\mathrm{M}-\mathrm{OH}]^{+} \mathrm{Calcd}$ for $\mathrm{C}_{21} \mathrm{H}_{19} \mathrm{BN}_{3} \mathrm{O}_{4} 388.1469$; Found 388.1474. UV-Vis (in $\left.\mathrm{CH}_{2} \mathrm{Cl}_{2}\right) \lambda[\mathrm{nm}]$ $\left(\varepsilon\left[\mathrm{M}^{-1} \mathrm{~cm}^{-1}\right]\right) 316$ (44048), 345 (41805), 399 (6049), 413 (4089), 527 (25676). Emission (in $\left.\mathrm{CH}_{2} \mathrm{Cl}_{2}\right) \lambda[\mathrm{nm}]\left(\phi_{\mathrm{f}}\right) 533(0.36)$.

2: Yield: $4 \mathrm{mg}(2 \%)$, m.p: $235.4-236.6{ }^{\circ} \mathrm{C} ;{ }^{1} \mathrm{H}$ NMR $\left(500 \mathrm{MHz}, \mathrm{CDCl}_{3}\right) \delta(\mathrm{ppm}) 7.92(\mathrm{~s}, 1 \mathrm{H}$, meso-H), $7.61(\mathrm{~d}, J=4.5 \mathrm{~Hz}, 2 \mathrm{H}, \beta-\mathrm{CH}), 7.48(\mathrm{~d}, J=4.5 \mathrm{~Hz}, 2 \mathrm{H}, \beta-\mathrm{CH}), 4.56(\mathrm{q}, J=5 \mathrm{~Hz}, 4 \mathrm{H}$, $\mathrm{CH}_{3} \mathrm{CH}_{2}-$ ), 4.07 (dd, $J=14.8 \mathrm{~Hz}$ and $18.8 \mathrm{~Hz}, 1 \mathrm{H}$, methylene), 4.03 (dd, $J=14.8 \mathrm{~Hz}$ and $18.8 \mathrm{~Hz}$, $1 \mathrm{H}$, methylene), $3.65(\mathrm{dd}, J=14.4 \mathrm{~Hz}$ and $18.0 \mathrm{~Hz}, 1 \mathrm{H}$, methylene), $3.62(\mathrm{dd}, J=14.4 \mathrm{~Hz}$ and $18.0 \mathrm{~Hz}, 1 \mathrm{H}$, methylene), $1.60\left(\mathrm{t}, \mathrm{J}=7.2 \mathrm{~Hz}, 6 \mathrm{H}, \mathrm{CH}_{3} \mathrm{CH}_{2}-\right) .{ }^{11} \mathrm{~B} \mathrm{NMR}\left(128 \mathrm{MHz}, \mathrm{CDCl}_{3}\right)$ 
$\left(\mathrm{BF}_{3} . \mathrm{OEt}_{2}\right.$ as internal standard) $\delta(\mathrm{ppm})-14.55 .{ }^{13} \mathrm{C} \mathrm{NMR}\left(125 \mathrm{MHz}, \mathrm{CDCl}_{3}\right) \delta(\mathrm{ppm}) 167.0,158.2$, 144.2, 134.7, 122.2, 116.0, 114.2, 101.3, 60.5, 32.9, 30.9, 14.7. HRMS (ESI-MS) m/z: $[\mathrm{M}+\mathrm{H}]^{+}$ Calcd for $\mathrm{C}_{42} \mathrm{H}_{38} \mathrm{~B}_{2} \mathrm{~N}_{6} \mathrm{O}_{9}$ 793.2959; Found 793.2967. UV-Vis (in $\left.\mathrm{CH}_{2} \mathrm{Cl}_{2}\right) \lambda[\mathrm{nm}]\left(\varepsilon\left[\mathrm{M}^{-1} \mathrm{~cm}^{-1}\right]\right)$ 312 (43478), 399 (4905), 418 (5182), 540 (15007). Emission (in $\left.\mathrm{CH}_{2} \mathrm{Cl}_{2}\right) \lambda$ [nm] ( $\left.\phi_{\mathrm{f}}\right) 616(0.14)$.

\section{2. c. Synthesis of $1 b \& 1 c:$}

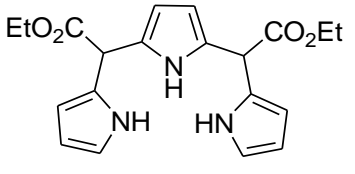

6

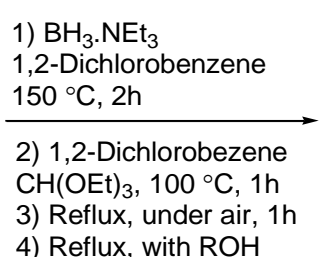

4) Reflux, with $\mathrm{ROH}$

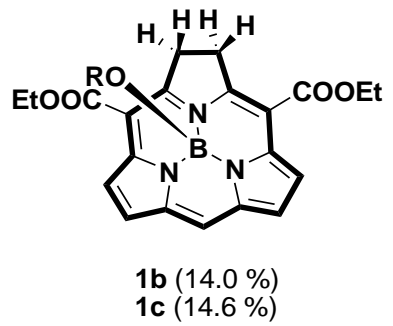

Freshly prepared diester tripyrrane $6(500 \mathrm{mg}, 1.35 \mathrm{mmol})$ and borane triethylamine complex (602 $\mu \mathrm{L}, 4.1 \mathrm{mmol})$ were added in freshly distilled 1,2-dichlorobenzene $(20 \mathrm{~mL})$ and kept under high vacuum for half an hour, and then the reaction mixture was heated in an oil bath at $150{ }^{\circ} \mathrm{C}$ for $2 \mathrm{~h}$ under nitrogen atmosphere. The reaction mixture was allowed to cool to $100{ }^{\circ} \mathrm{C}$ and was diluted with 1,2-dichlorobenzene $(100 \mathrm{~mL})$ containing triethylorthoformate $(3.0 \mathrm{~mL}, 27.41 \mathrm{mmol})$ and the reaction mixture was heated at $100{ }^{\circ} \mathrm{C}$ for $1 \mathrm{~h}$ in a nitrogen atmosphere. Subsequently, nitrogen atmosphere was removed and the reaction mixture was heated at reflux under an open air atmosphere for $1 \mathrm{~h}$. 1,2-dichlorobenzene was removed in vacuum and the black residue was dissolved in chloroform/ methanol or ethanol $(100 \mathrm{~mL}, 1: 1)$ mixture and was refluxed for $6 \mathrm{~h}$. The solvent was evaporated, and the crude product was passed through silica gel column, and the desired subchlorin was eluted by EtOAc/ Hexane as an orange-red solution with green fluorescence. The solvent was removed under reduced pressure and dried to afford red solid subchlorin.

1b: Yield: $80 \mathrm{mg}(14 \%){ }^{1} \mathrm{H}$ NMR (500MHz, $\left.\mathrm{CDCl}_{3}\right) \delta(\mathrm{ppm}) 8.41(\mathrm{~s}, 1 \mathrm{H}$, meso-H), $7.92(\mathrm{~d}, J=$ $4.5 \mathrm{~Hz}, 2 \mathrm{H}, \beta-\mathrm{CH}$ ), 7.81 (d, $J=4.5 \mathrm{~Hz}, 2 \mathrm{H}, \beta-\mathrm{CH}$ ), 4.57 (q, $J=7 \mathrm{~Hz}, 4 \mathrm{H}, \mathrm{CH}_{3} \mathrm{CH}_{2}-$ ), 4.45 (dd, $J$ $=15.0 \mathrm{~Hz}$ and $20.5 \mathrm{~Hz}, 1 \mathrm{H}$, methylene), $4.42(\mathrm{dd}, J=15.0 \mathrm{~Hz}$ and $20.5 \mathrm{~Hz}, 1 \mathrm{H}$, methylene), 4.02 (dd, $J=15.0 \mathrm{~Hz}$ and $20.5 \mathrm{~Hz}, 1 \mathrm{H}$, methylene), 3.99 (dd, $J=15.0 \mathrm{~Hz}$ and $20.5 \mathrm{~Hz}, 1 \mathrm{H}$, methylene), $1.56(\mathrm{t}, \mathrm{J}=7 \mathrm{~Hz}, 6 \mathrm{H}, \mathrm{CH} 3 \mathrm{CH} 2-) .1 .521$ (s, 3H, axial $-\mathrm{OMe}) ;{ }^{11} \mathrm{~B} \mathrm{NMR}\left(128 \mathrm{MHz}, \mathrm{CDCl}_{3}\right)$ $\left(\mathrm{BF}_{3} . \mathrm{OEt}_{2}\right.$ as internal standard) $\delta(\mathrm{ppm})-11.98 .{ }^{13} \mathrm{C} \mathrm{NMR}\left(125 \mathrm{MHz}, \mathrm{CDCl}_{3}\right) \delta(\mathrm{ppm}) 166.8,159.2$, 144.8, 135.6, 123.4, 117.5, 115.0, 102.5, 60.9, 47.8, 33.5, 14.6. HRMS (ESI-MS) m/z: $\left[\mathrm{M}+\mathrm{Na}^{+}\right.$ Calcd for $\mathrm{C}_{22} \mathrm{H}_{22} \mathrm{BN}_{3} \mathrm{O}_{5} \mathrm{Na}$ 442.1550; Found 442.1545. UV-Vis (in $\left.\mathrm{CH}_{2} \mathrm{Cl}_{2}\right) \lambda[\mathrm{nm}]\left(\varepsilon\left[\mathrm{M}^{-1} \mathrm{~cm}^{-1}\right]\right)$ 314 (44888), 343 (37562), 397 (4528), 412 (2279), 525 (30044). Emission (in $\left.\mathrm{CH}_{2} \mathrm{Cl}_{2}\right) \lambda[\mathrm{nm}]\left(\phi_{\mathrm{f}}\right)$ $534(0.14)$.

1c: Yield: $86 \mathrm{mg}(15 \%){ }^{1} \mathrm{H}$ NMR (500MHz, $\left.\mathrm{CDCl}_{3}\right) \delta(\mathrm{ppm}) 8.42(\mathrm{~s}, 1 \mathrm{H}$, meso-H), $7.93(\mathrm{~d}, J=$ $4.5 \mathrm{~Hz}, 2 \mathrm{H}, \beta-\mathrm{CH}), 7.82(\mathrm{~d}, J=4.5 \mathrm{~Hz}, 2 \mathrm{H}, \beta-\mathrm{CH}), 4.60$ (q, $\left.J=5 \mathrm{~Hz}, 4 \mathrm{H}, \mathrm{CH}_{3} \mathrm{CH}_{2}-\right), 4.46$ (dd, $J$ $=15.0 \mathrm{~Hz}$ and $20.0 \mathrm{~Hz}, 1 \mathrm{H}$, methylene), $4.43(\mathrm{dd}, J=15.0 \mathrm{~Hz}$ and $20.0 \mathrm{~Hz}, 1 \mathrm{H}$, methylene), 4.04 (dd, $J=15.0 \mathrm{~Hz}$ and $20.5 \mathrm{~Hz}, 1 \mathrm{H}$, methylene), $4.00(\mathrm{dd}, J=15.0 \mathrm{~Hz}$ and $20.5 \mathrm{~Hz}, 1 \mathrm{H}$, methylene), 
$1.60\left(\mathrm{~m}, 8 \mathrm{H}\right.$, ester $\mathrm{CH}_{3} \mathrm{CH}_{2}$ ). $0.18\left(\mathrm{t}, J=7 \mathrm{~Hz}, 3 \mathrm{H}\right.$, axial $\left.-\mathrm{OCH}_{2} \mathrm{CH}_{3}\right) ;{ }^{11} \mathrm{~B}$ NMR (192 MHz, $\left.\mathrm{CDCl}_{3}\right)\left(\mathrm{BF}_{3} . \mathrm{OEt}_{2}\right.$ as internal standard) $\delta(\mathrm{ppm})-12.63 .{ }^{13} \mathrm{C} \mathrm{NMR}\left(125 \mathrm{MHz}, \mathrm{CDCl}_{3}\right) \delta(\mathrm{ppm})$ $166.9,159.4,144.9,135.6,123.3,117.3,115.0,102.5,60.8,55.4,33.4,14.6$. HRMS (ESI-MS)

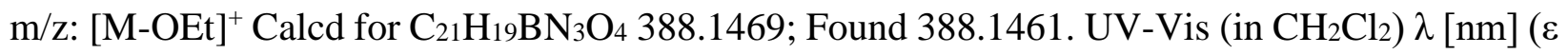
$\left[\mathrm{M}^{-1} \mathrm{~cm}^{-1}\right]$ ) 314 (44188), 343 (37308), 397 (4498), 411 (2293), 525 (29841). Emission (in $\mathrm{CH}_{2} \mathrm{Cl}_{2}$ ) $\lambda[\mathrm{nm}](\phi \mathrm{f}) 533(0.13)$.

\section{2. d. Synthesis of 2 (Subchlorin $\mu$ oxo dimer) from 1a (axial hydroxyl subchlorin monomer):}

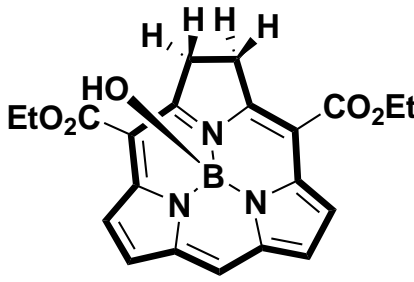

1a

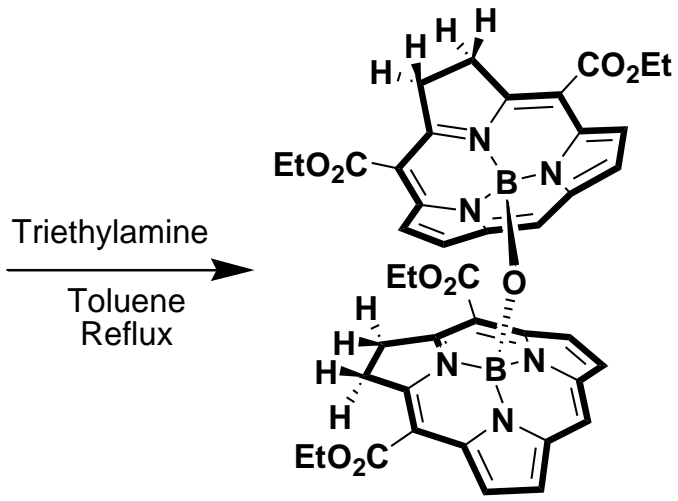

2 (61.0\%)

Axially hydroxyl subchlorin 1a was treated with dry triethylamine in freshly distilled dry toluene medium. The reaction mixture is heated in an oil bath and refluxed for one hour under nitrogen atmosphere to obtain the dimer $\mathbf{2}$ in $61 \%$ yield.

\section{2. e. Interconversion of subchlorin to subporphyrin:}
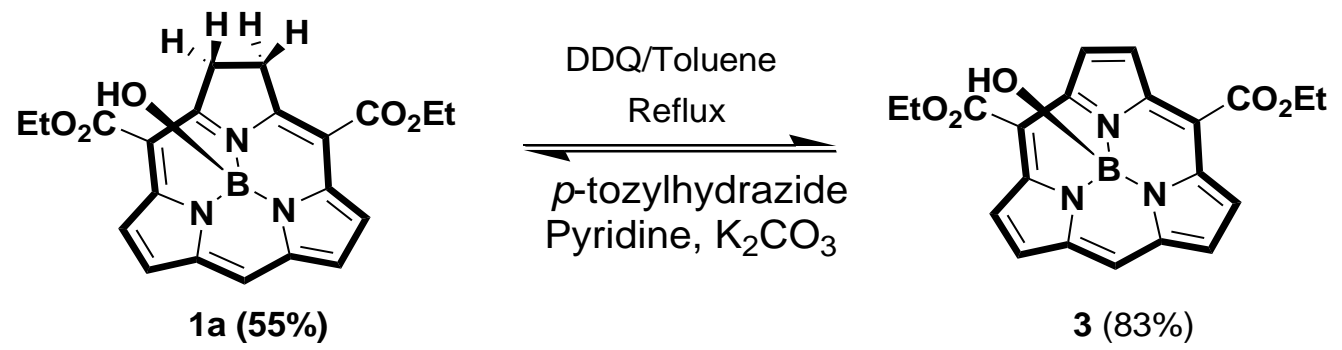

Subchlorin 1a $(22.0 \mathrm{mg}, 53 \mu \mathrm{mol})$ and DDQ $(34.0 \mathrm{mg}, 131 \mu \mathrm{mol})$ were dissolved in toluene $(5.0$ $\mathrm{mL}$ ), and the reaction mixture was heated in an oil bath at refluxed for overnight. The reaction mixture was cooled to room temperature, and toluene was removed under pressure. Crude was passed through aa pad of neutral alumina, and the oxidized subporphyrin was eluted with EtOAc/ Hexane (1:1) as a yellowish-orange solution with green fluorescence.

Yield: $18 \mathrm{mg}$ (83 \%), m.p: 245.3 - $246.5{ }^{\circ} \mathrm{C} ;{ }^{1} \mathrm{H}$ NMR $\left(500 \mathrm{MHz}, \mathrm{CDCl}_{3}\right) \delta(\mathrm{ppm}) 9.04(\mathrm{~s}, 1 \mathrm{H}$, meso-H), 8.76 (s, $2 \mathrm{H}, \beta-\mathrm{CH}), 8.68(\mathrm{~d}, J=4.5 \mathrm{~Hz}, 2 \mathrm{H}, \beta-\mathrm{CH}), 8.18(\mathrm{~d}, J=4.5 \mathrm{~Hz}, 2 \mathrm{H}, \beta-\mathrm{CH}), 4.80$ (q, $\left.J=5 \mathrm{~Hz}, 4 \mathrm{H}, \mathrm{CH}_{3} \mathrm{CH}_{2}-\right), 1.71\left(\mathrm{t}, \mathrm{J}=7.2 \mathrm{~Hz}, 6 \mathrm{H}, \mathrm{CH}_{3} \mathrm{CH}_{2}-\right),-2.65$, (s, $1 \mathrm{H}$, axial $\left.-\mathrm{OH}\right) ;{ }^{11} \mathrm{~B}$ NMR $\left(128 \mathrm{MHz}, \mathrm{CDCl}_{3}\right)\left(\mathrm{BF}_{3} . \mathrm{OEt}_{2}\right.$ as internal standard) $\delta(\mathrm{ppm})-15.85 .{ }^{13} \mathrm{C} \mathrm{NMR}(125 \mathrm{MHz}$, 
$\left.\mathrm{CDCl}_{3}\right) \delta(\mathrm{ppm}) 166.7,145.1,144.8,141.8,125.1,123.6,123.3,109.5,107.3,61.7,46.5,12.6$. HRMS (ESI-MS) m/z: [M-OH] ${ }^{+}$Calcd for $\mathrm{C}_{21} \mathrm{H}_{17} \mathrm{BN}_{3} \mathrm{O}_{4}$ 386.1312; Found 386.1311. UV-Vis (in $\left.\mathrm{CH}_{2} \mathrm{Cl}_{2}\right) \lambda[\mathrm{nm}]\left(\varepsilon\left[\mathrm{M}^{-1} \mathrm{~cm}^{-1}\right]\right) 362$ (66269), 476 (2995), 505 (5795). Emission: (in $\left.\mathrm{CH}_{2} \mathrm{Cl}_{2}\right) \lambda[\mathrm{nm}]$ 523.

Subporphyrin $\mathbf{3}(10 \mathrm{mg}, 25 \mu \mathrm{mol})$ in pyridine $(2 \mathrm{~mL})$ was treated with $p$-toluenesulfonyl hydrazide $(10 \mathrm{mg}, 54 \mu \mathrm{mol})$ and potassium carbonate $(17 \mathrm{mg}, 124 \mu \mathrm{mol})$. The reaction mixture was heated to reflux under $\mathrm{N}_{2}$ atmosphere. Additional p-tosylhydrazide (2 eq.) and potassium carbonate (5 eq.) were added in an hour interval. After consumption of subporphyrin, the solvent was evaporated under reduced pressure. The residue was dissolved in dichloromethane, washed with water and dried over sodium sulphate. Crude product was passed through a pad of neutral alumina and subchlorin 1a was collected as a orange-red solution with intense green fluorescence in 55\% yield. 


\section{NMR ANALYSIS}

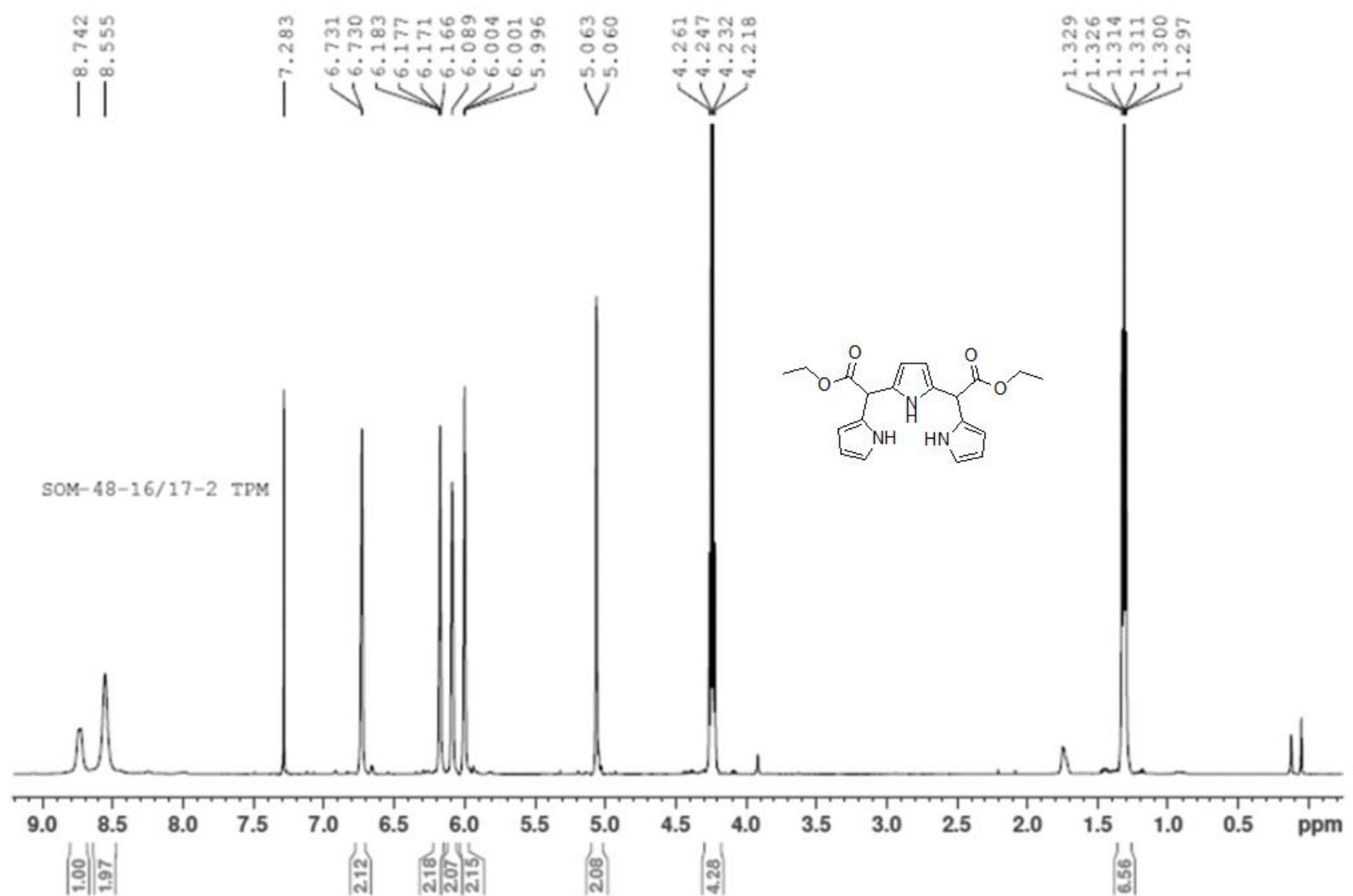

Figure S1: ${ }^{1} \mathrm{H}$ NMR spectrum of 6 in $\mathrm{CDCl}_{3}(400 \mathrm{MHz})$. 

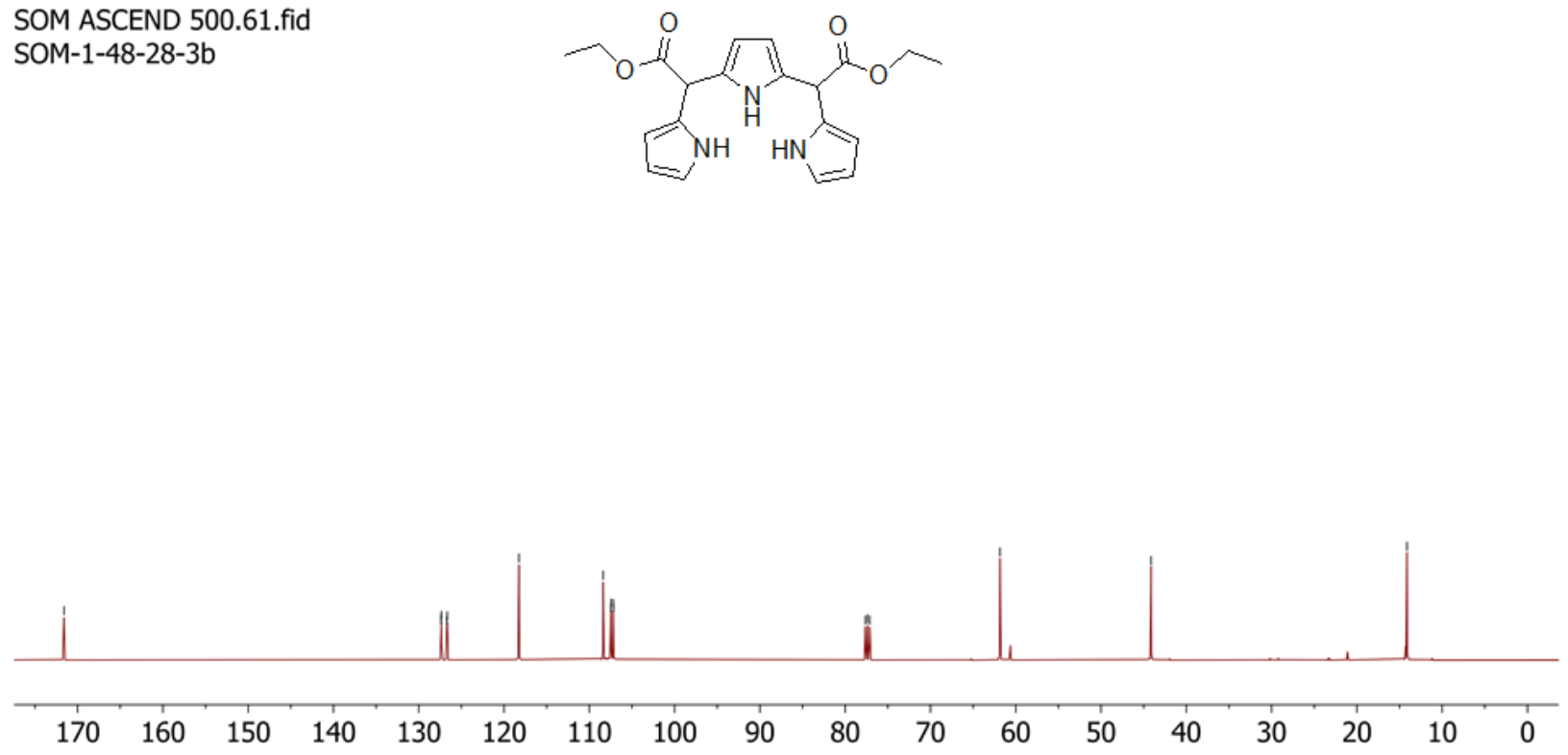

Figure S2: ${ }^{13} \mathrm{C}$ NMR spectrum of 6 in $\mathrm{CDCl}_{3}(125 \mathrm{MHz})$ 

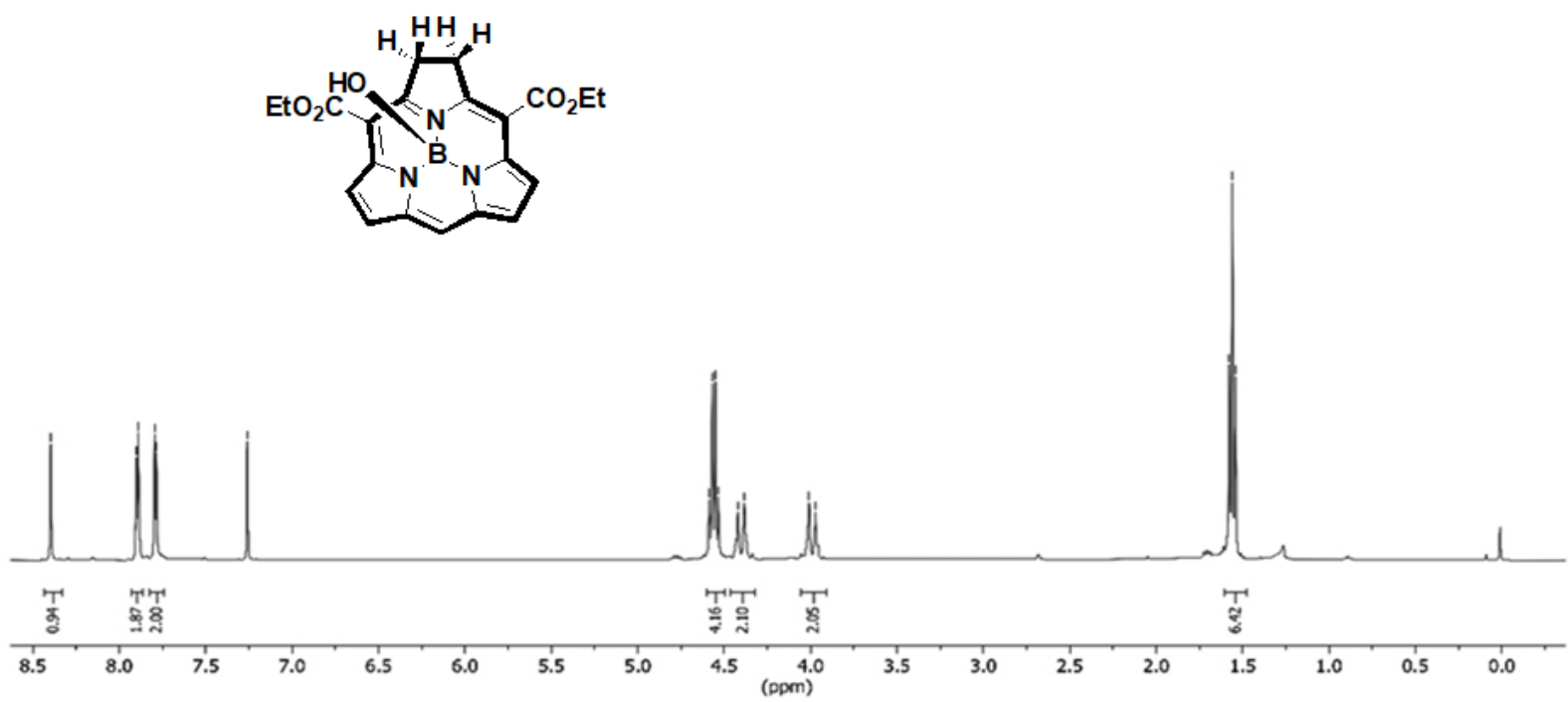

Figure S3: ${ }^{1} \mathrm{H}$ NMR spectrum of 1 a in $\mathrm{CDCl}_{3}(400 \mathrm{MHz})$. 


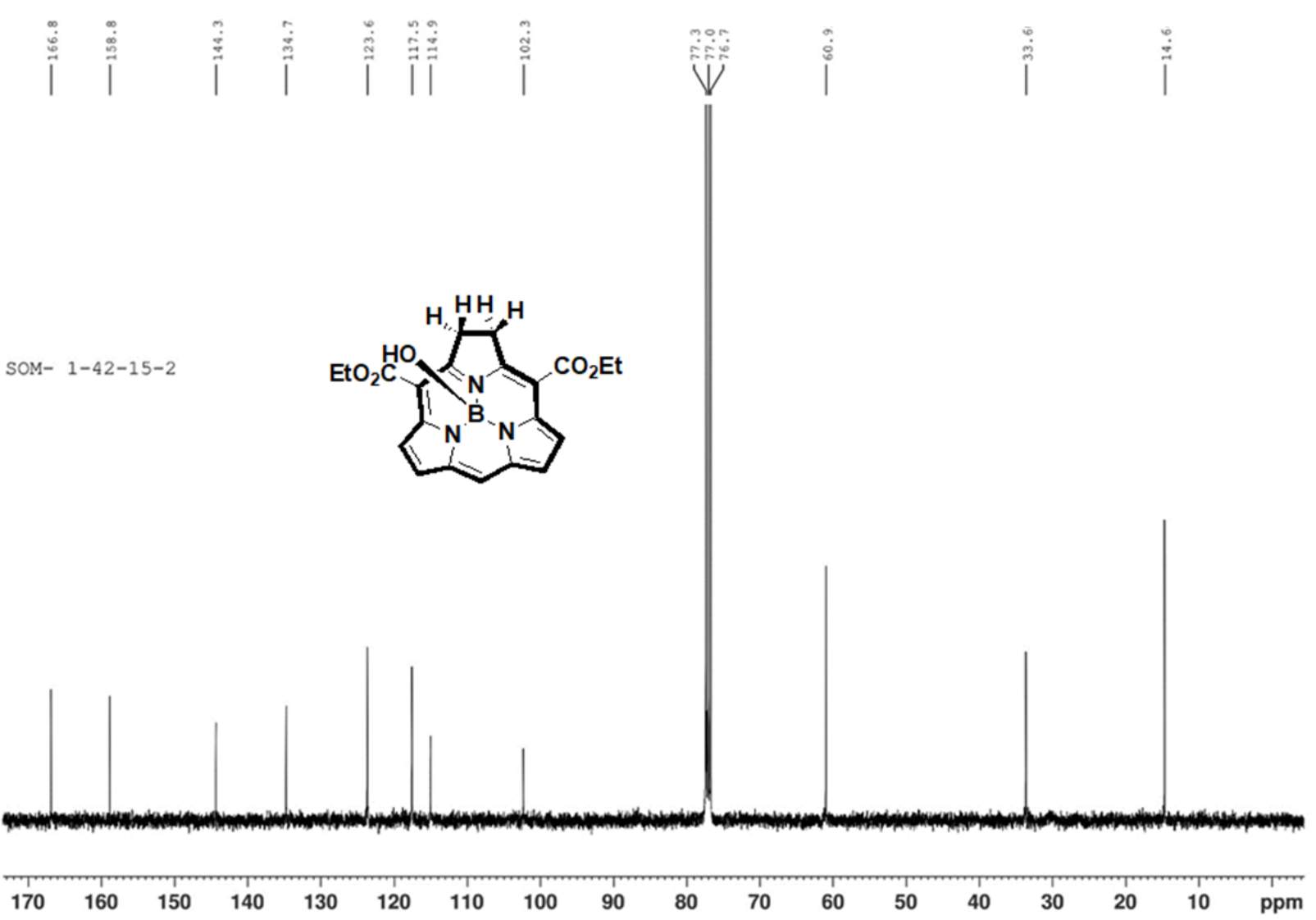

Figure S4: ${ }^{13} \mathrm{C}$ NMR spectrum of $\mathbf{1 a}$ in $\mathrm{CDCl}_{3}(100 \mathrm{MHz})$

$$
\text { बे }
$$

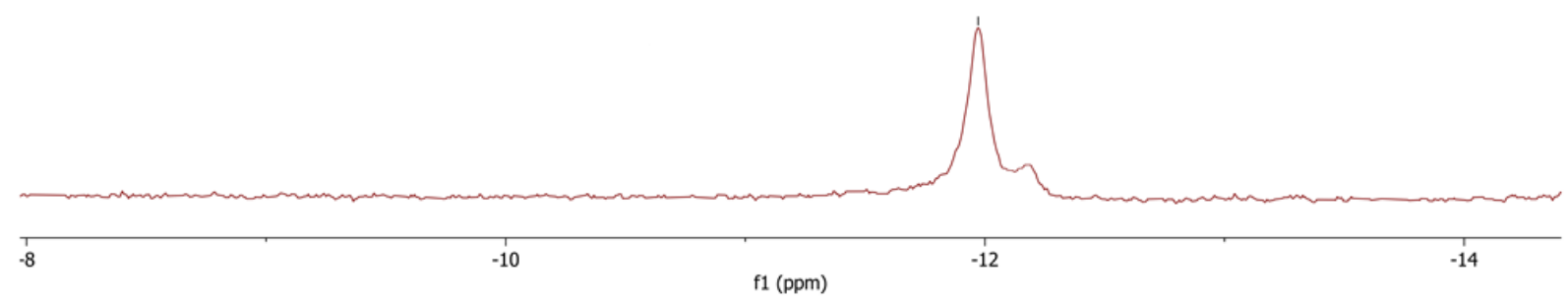

Figure S5: ${ }^{11} \mathrm{~B}$ NMR spectrum of Subchlorin 1a in $\mathrm{CDCl}_{3}(128 \mathrm{MHz})$. 
Variable temperature ${ }^{1} \mathrm{H}$ NMR study of 1a:

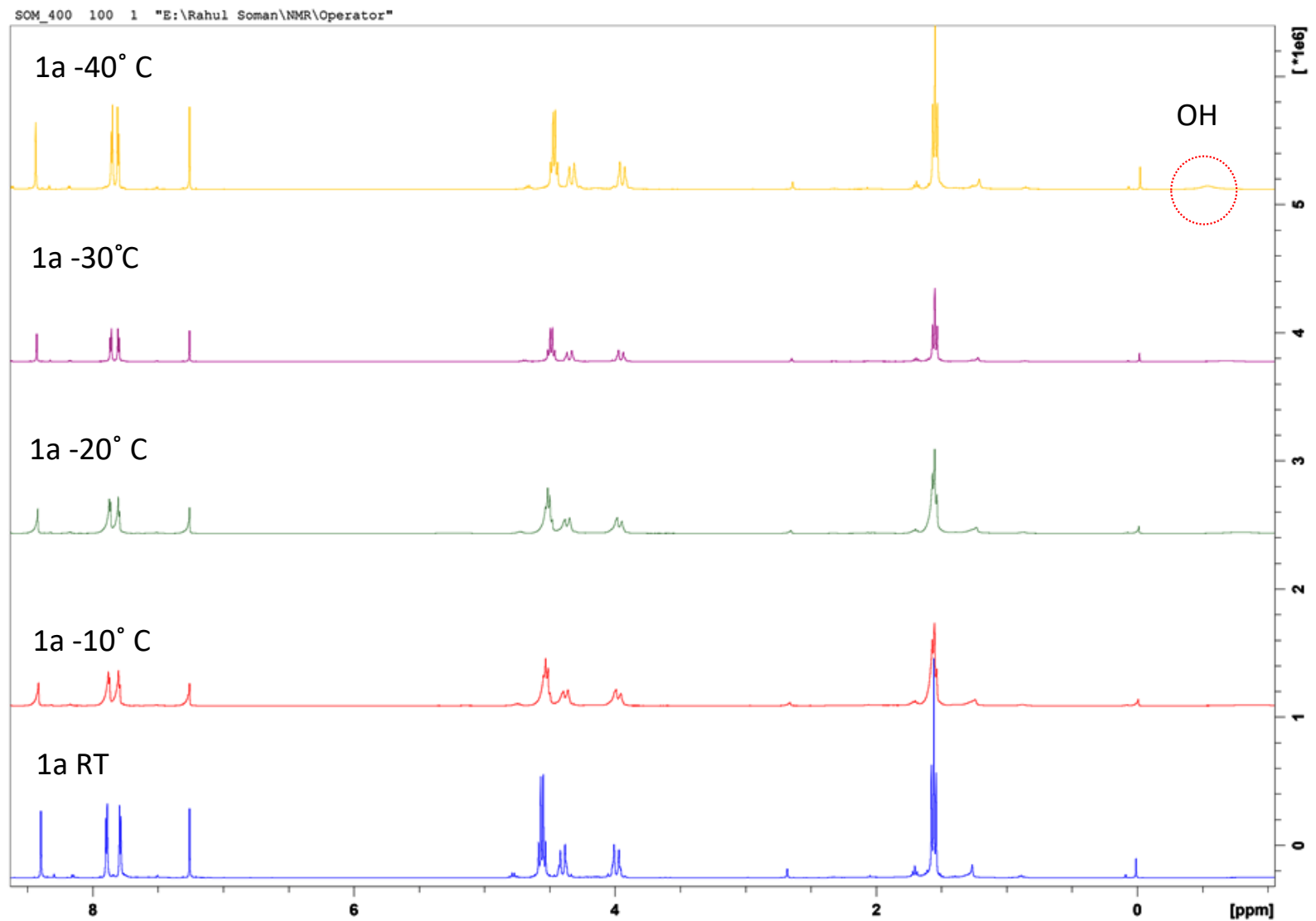

Figure S6: Variable temperature ${ }^{1} \mathrm{H}$ NMR spectrum of subchlorin 1a in $\mathrm{CDCl}_{3}$ (axial $\mathrm{OH}$ signal appears at $\left.-40^{\circ} \mathrm{C}\right)(400 \mathrm{MHz})$. 


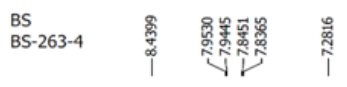
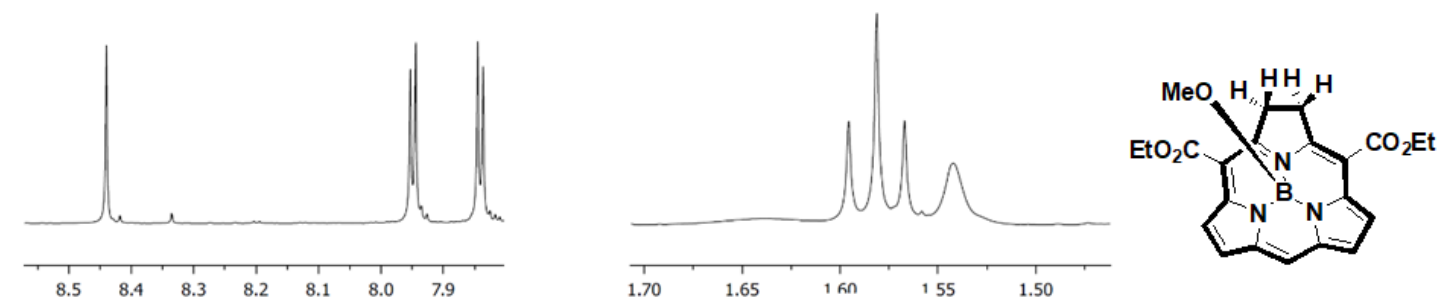

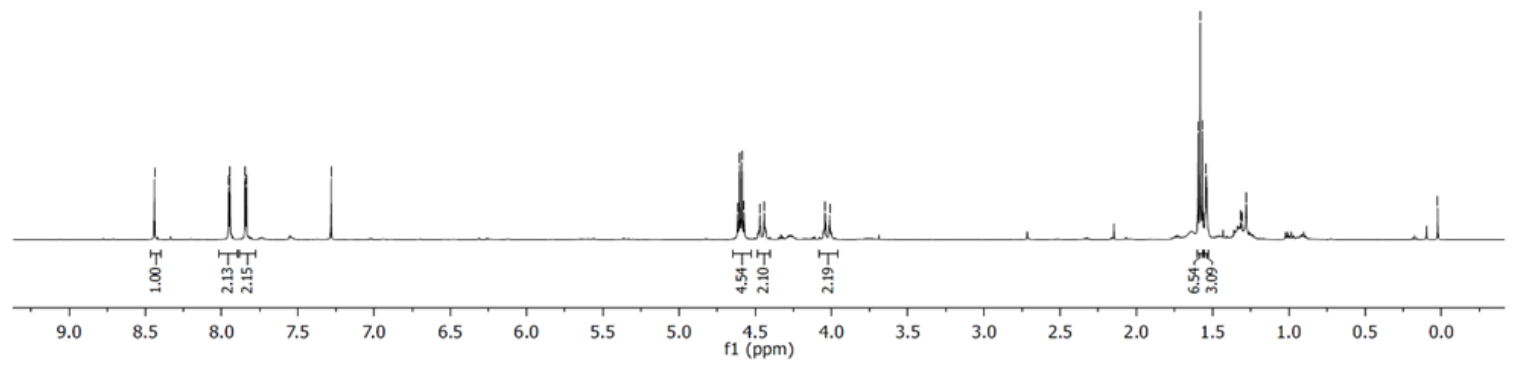

Figure S7: ${ }^{1} \mathrm{H}$ NMR spectrum of $\mathbf{1 b}$ in $\mathrm{CDCl}_{3}(500 \mathrm{MHz})$.

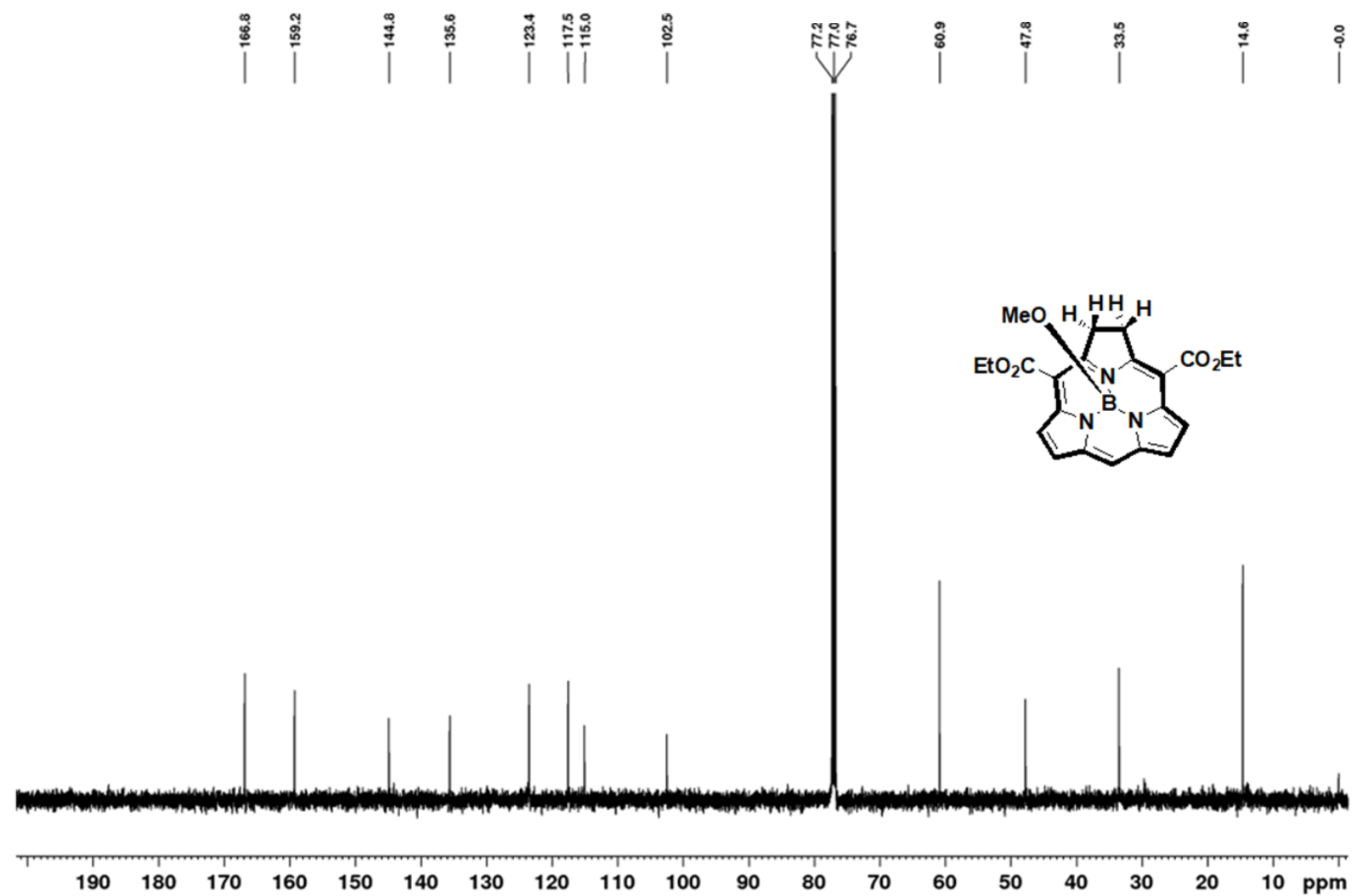

Figure S8: ${ }^{13} \mathrm{C}$ NMR spectrum of $\mathbf{1 b}$ in $\mathrm{CDCl}_{3}(125 \mathrm{MHz})$. 


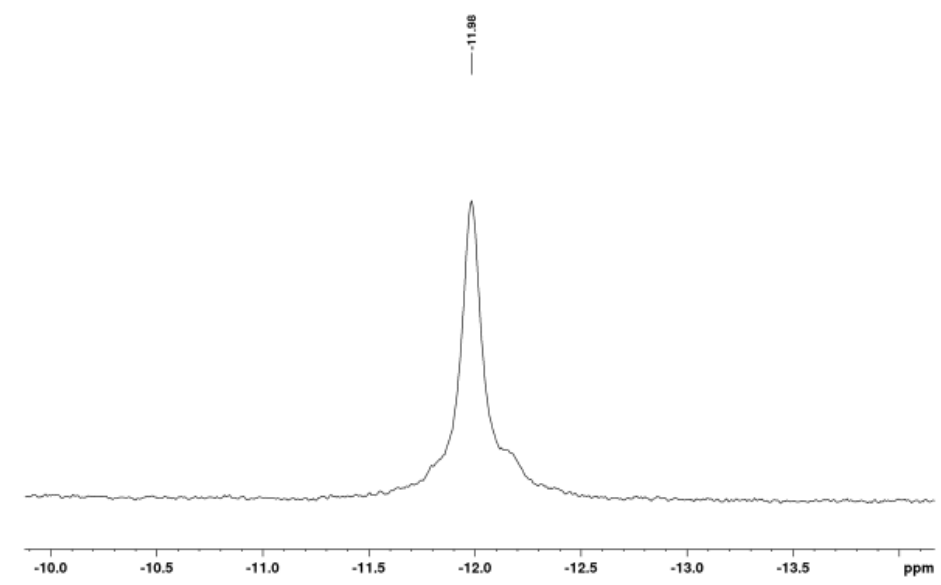

Figure S9: ${ }^{11} \mathrm{~B}$ NMR spectrum of subchlorin $\mathbf{1 b}$ in $\mathrm{CDCl}_{3}(128 \mathrm{MHz})$.

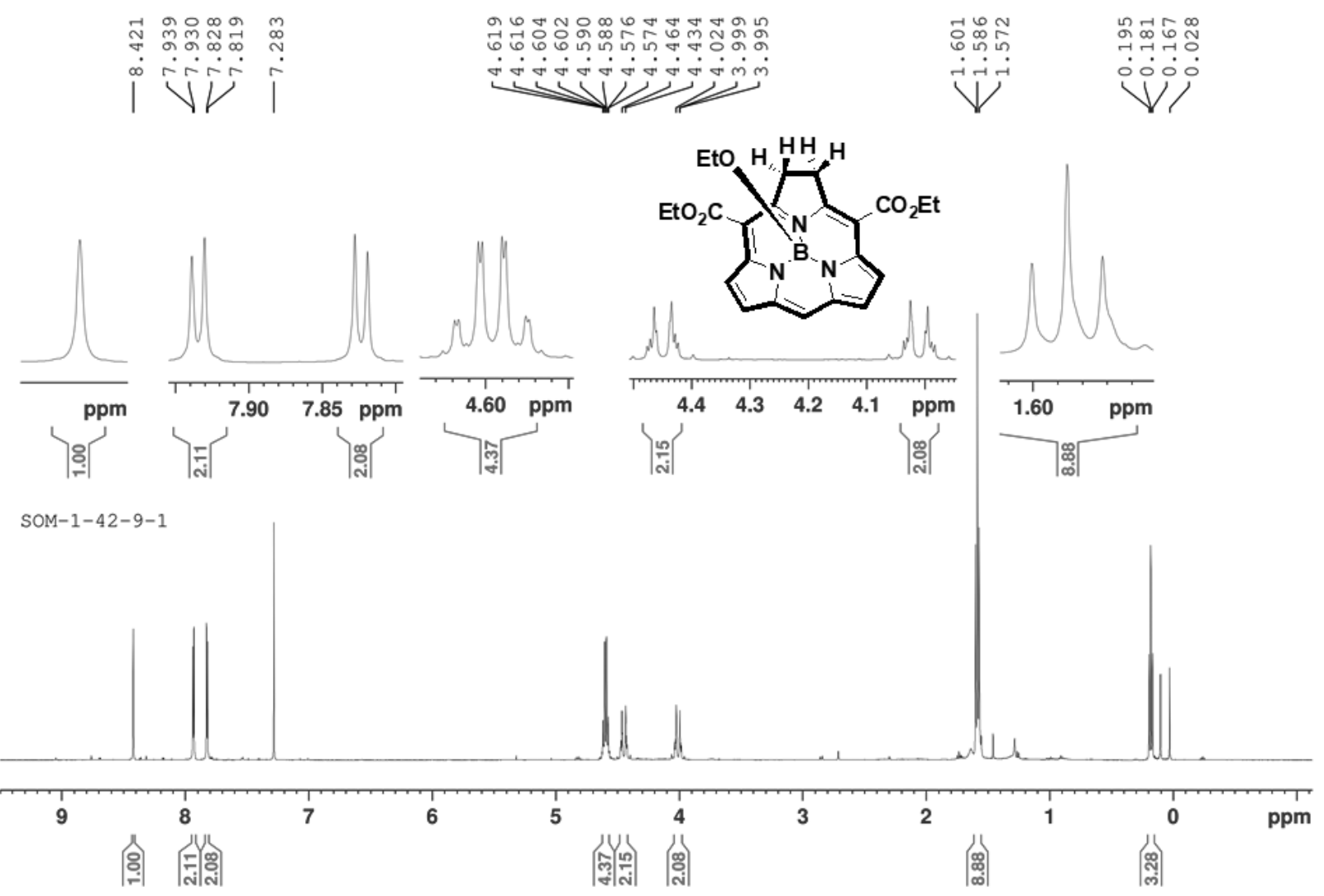

Figure S10: ${ }^{1} \mathrm{H}$ NMR spectrum of subchlorin $1 \mathrm{c}$ in $\mathrm{CDCl}_{3}(500 \mathrm{MHz})$. 


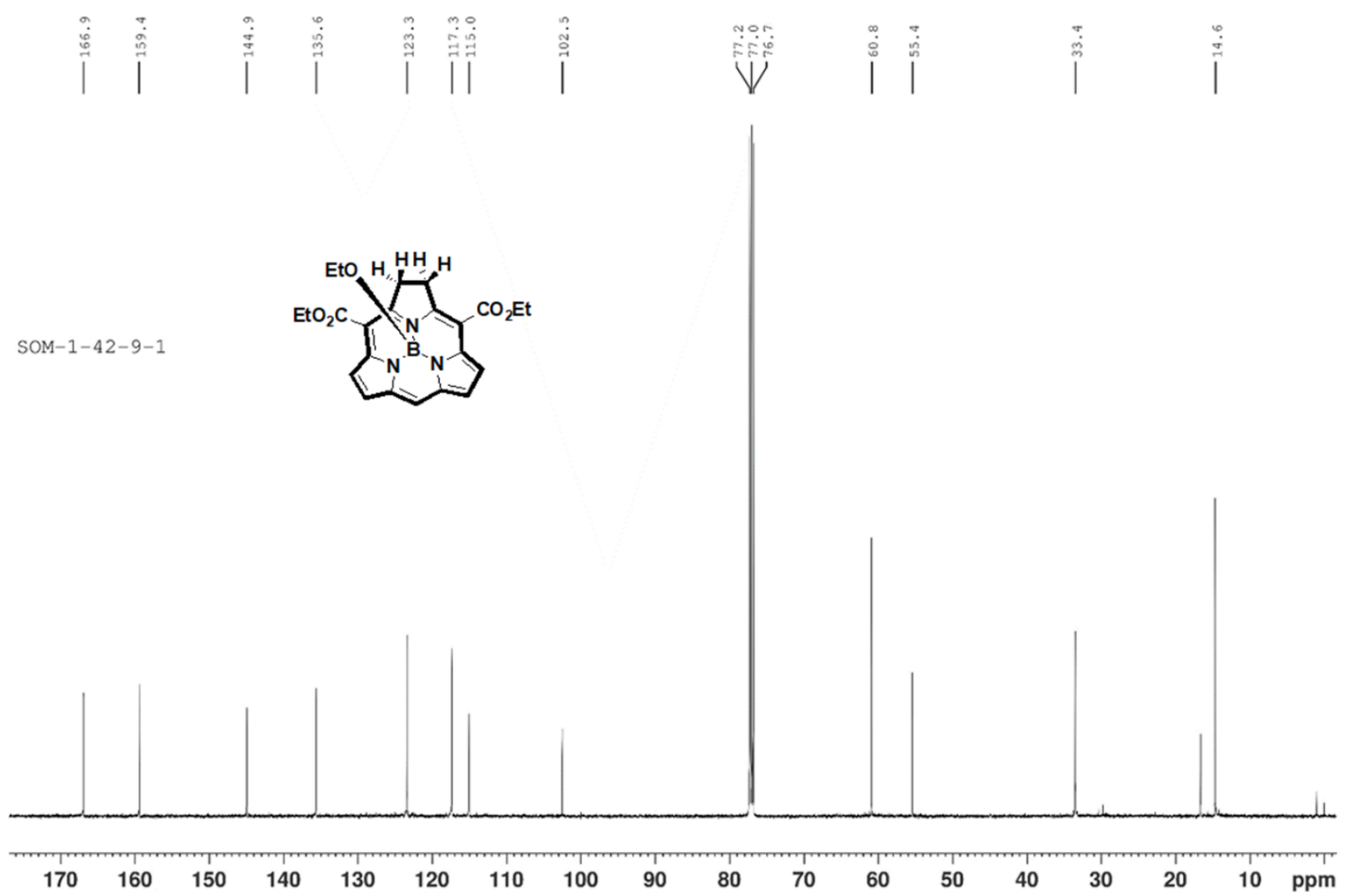

Figure S11: ${ }^{13} \mathrm{C}$ NMR spectrum of subchlorin 1c in $\mathrm{CDCl}_{3}(125 \mathrm{MHz})$.

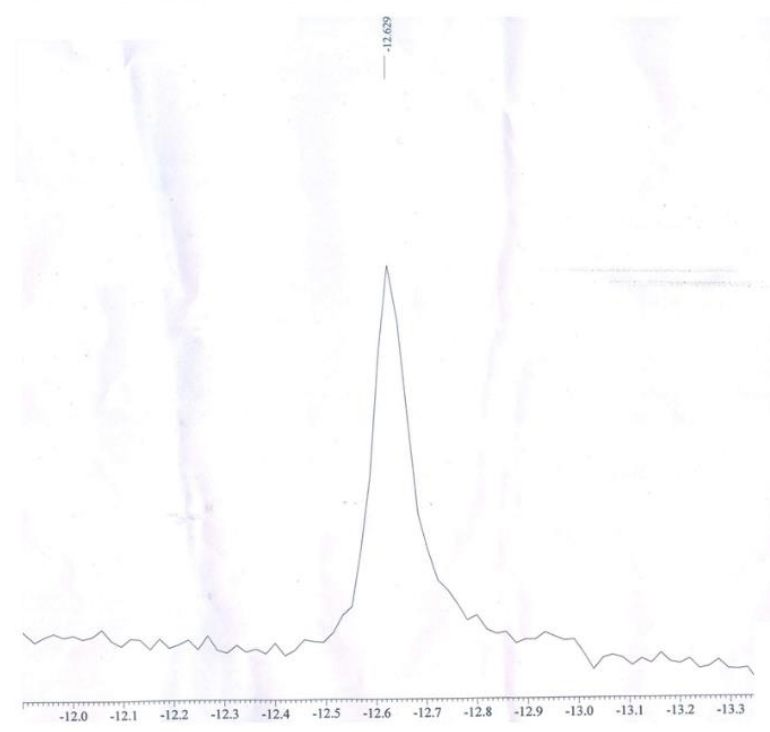

Figure S12: ${ }^{11} \mathrm{~B}$ NMR spectrum of subchlorin $1 \mathrm{c}$ in $\mathrm{CDCl}_{3}(192 \mathrm{MHz})$. 


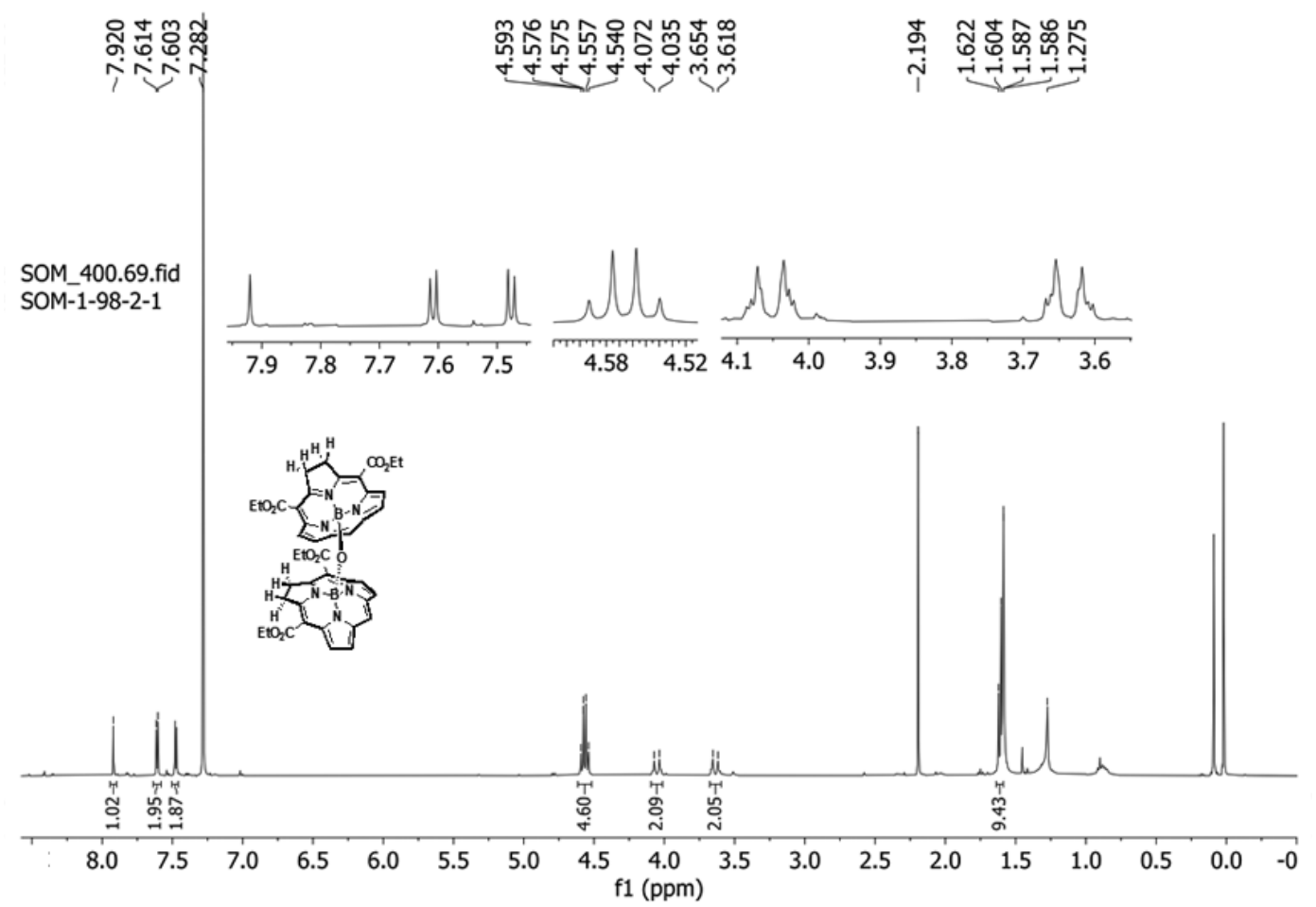

Figure S13: ${ }^{1} \mathrm{H}$ NMR spectrum of dimer 2 in $\mathrm{CDCl}_{3}(500 \mathrm{MHz})$.
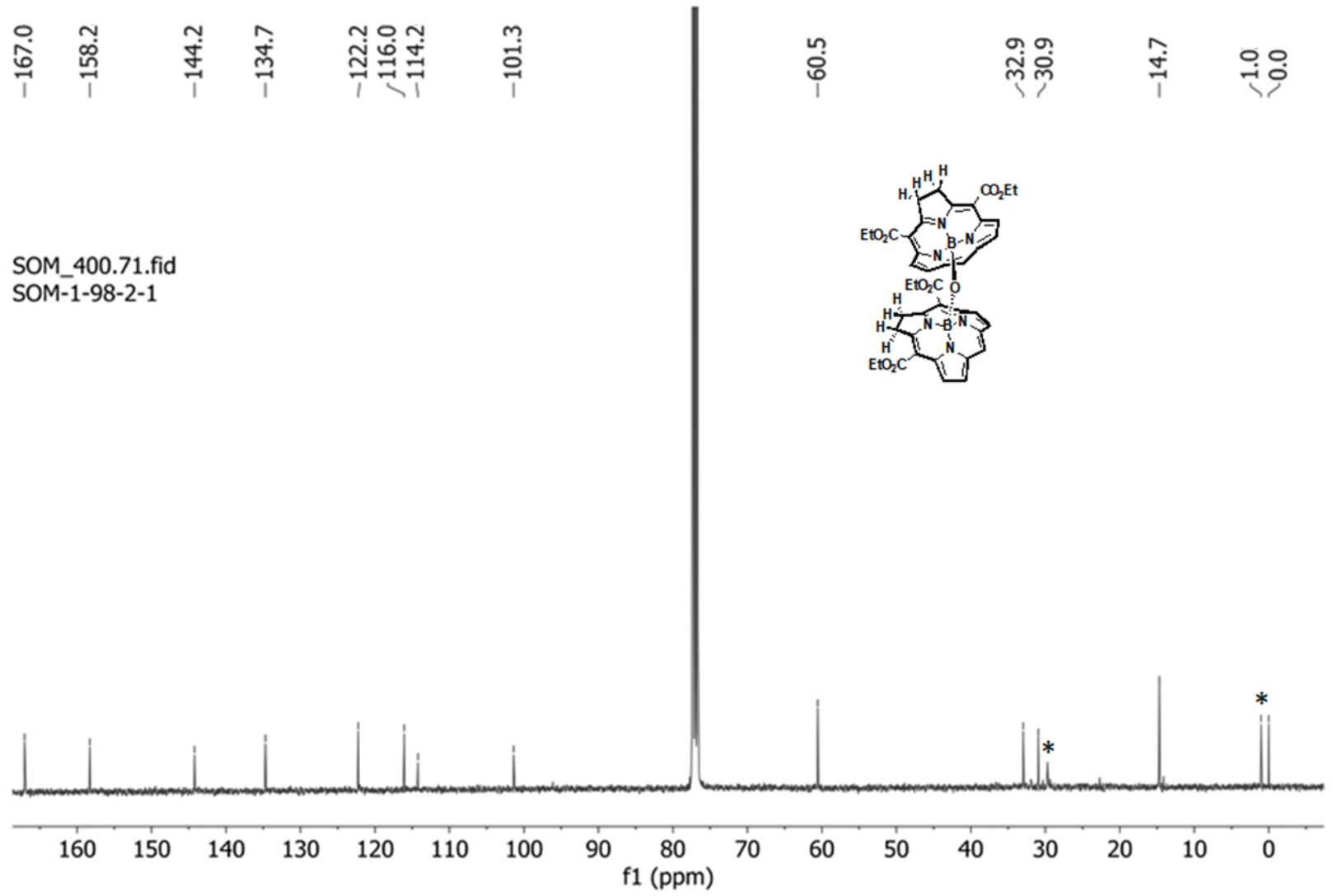

Figure S14: ${ }^{13} \mathrm{C}$ NMR spectrum of dimer 2 in $\mathrm{CDCl}_{3}(125 \mathrm{MHz})$. 


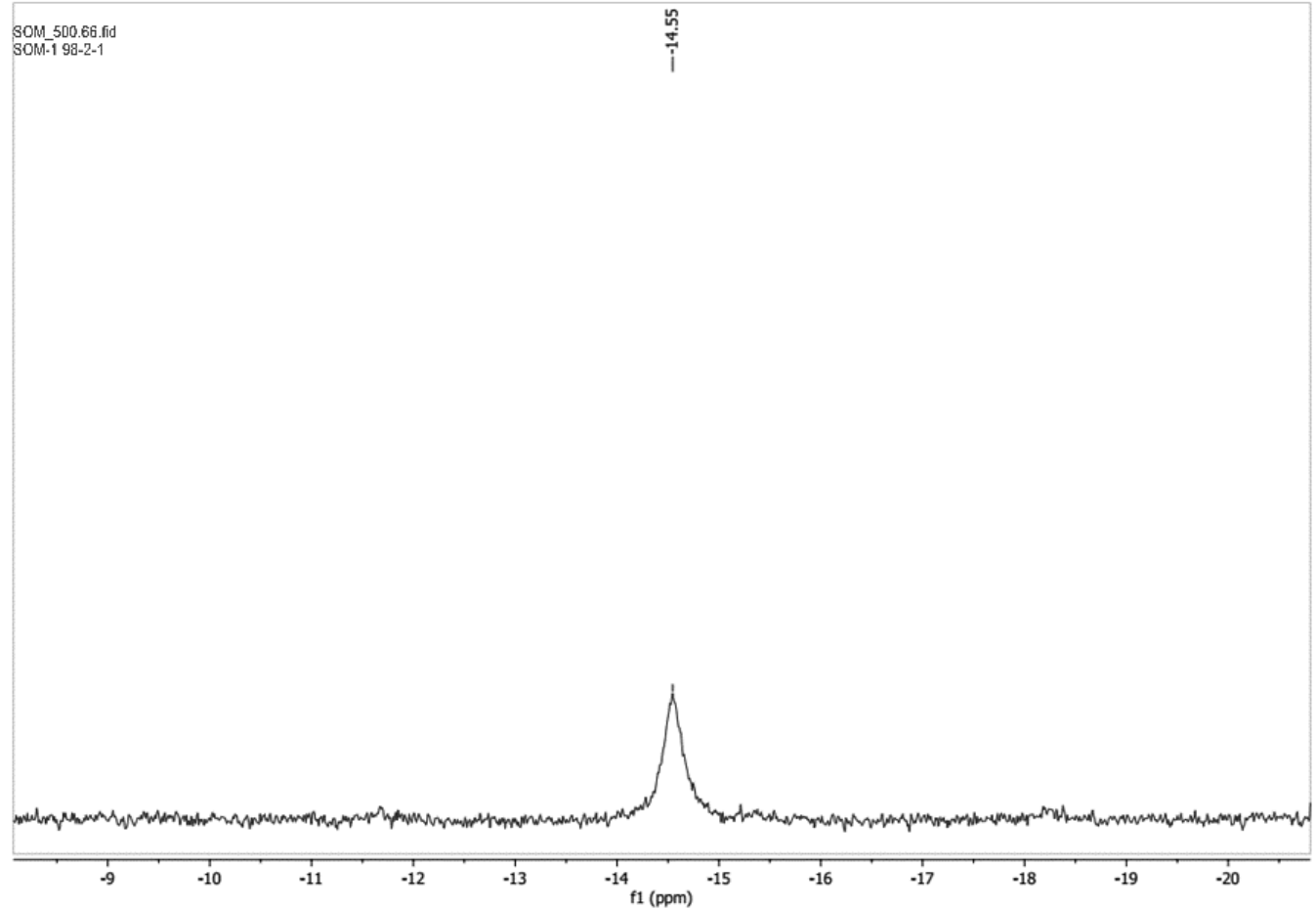

Figure S15: ${ }^{11} \mathrm{~B}$ NMR spectrum of subchlorin dimer 2 in $\mathrm{CDCl}_{3}(128 \mathrm{MHz})$. 


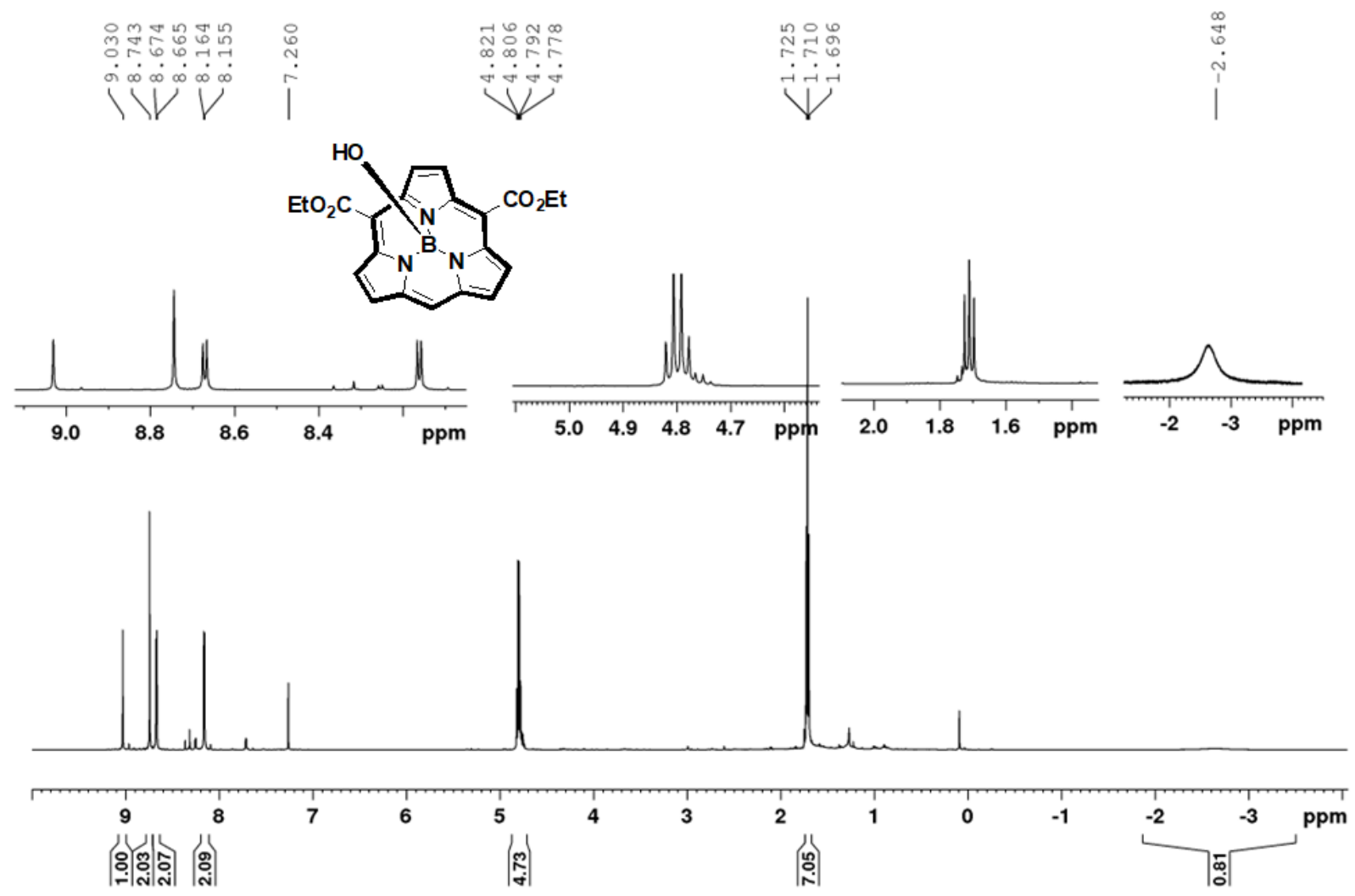

Figure S16: ${ }^{1} \mathrm{H}$ NMR spectrum of subporphyrin 3 in $\mathrm{CDCl}_{3}(500 \mathrm{MHz})$. 


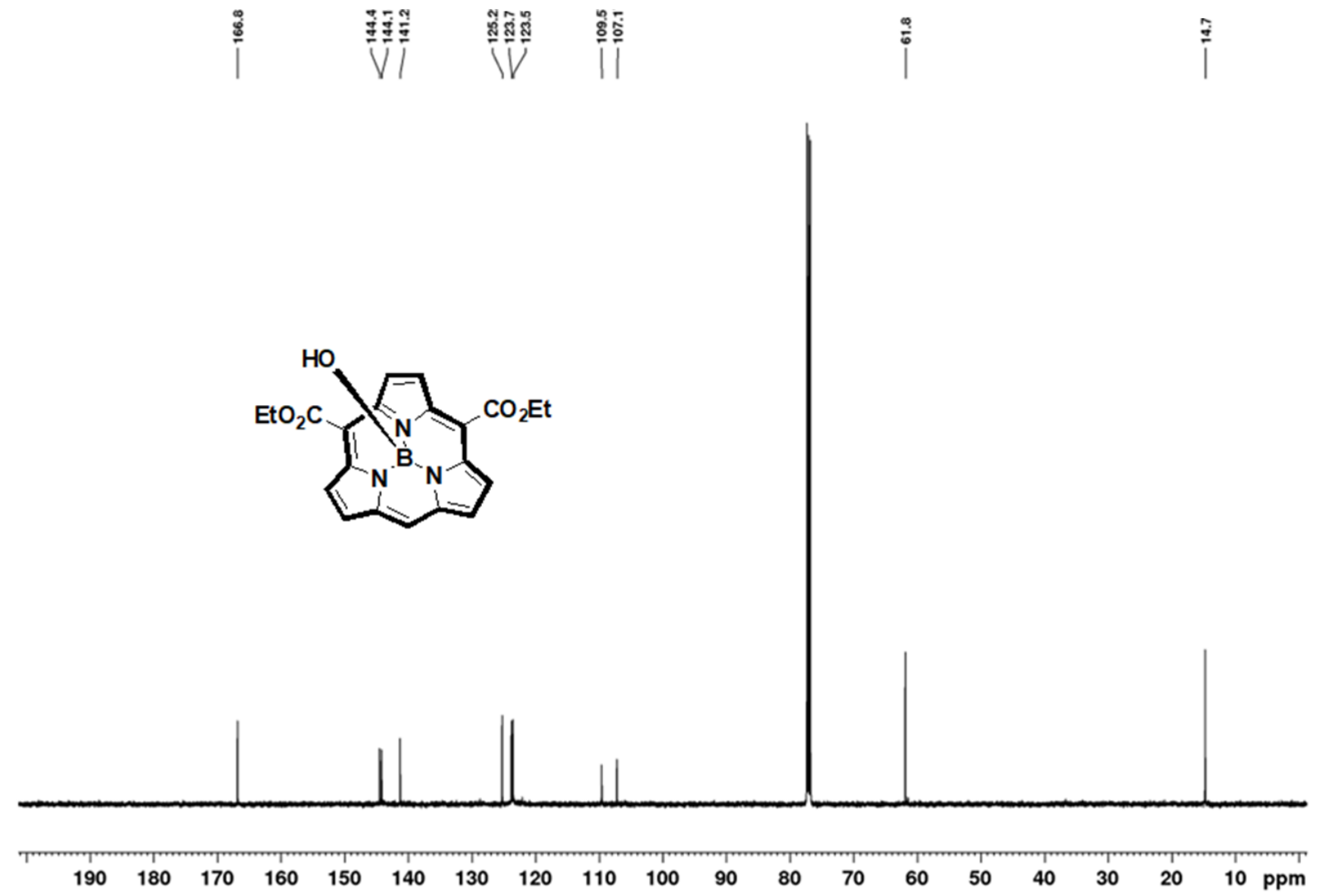

Figure S17: ${ }^{13} \mathrm{C}$ NMR spectrum of subporphyrin 3 in $\mathrm{CDCl}_{3}(125 \mathrm{MHz})$.

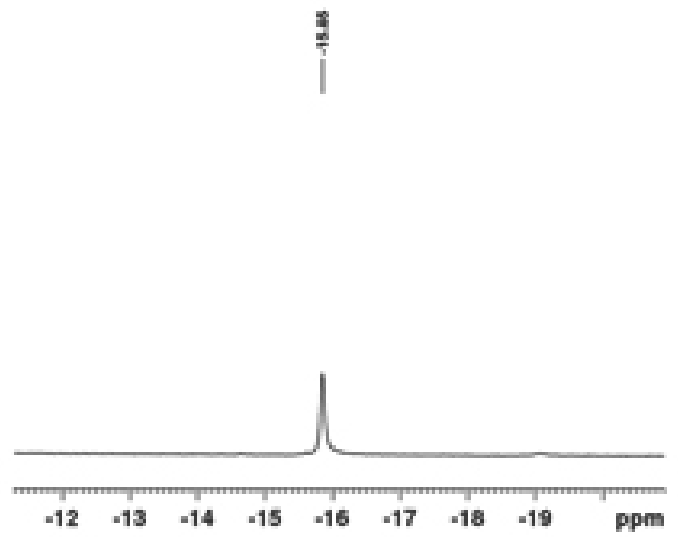

Figure S18: ${ }^{11} \mathrm{~B}$ NMR spectrum of subporphyrin 3 in $\mathrm{CDCl}_{3}(128 \mathrm{MHz})$. 


\section{HRMS ANALYSIS}

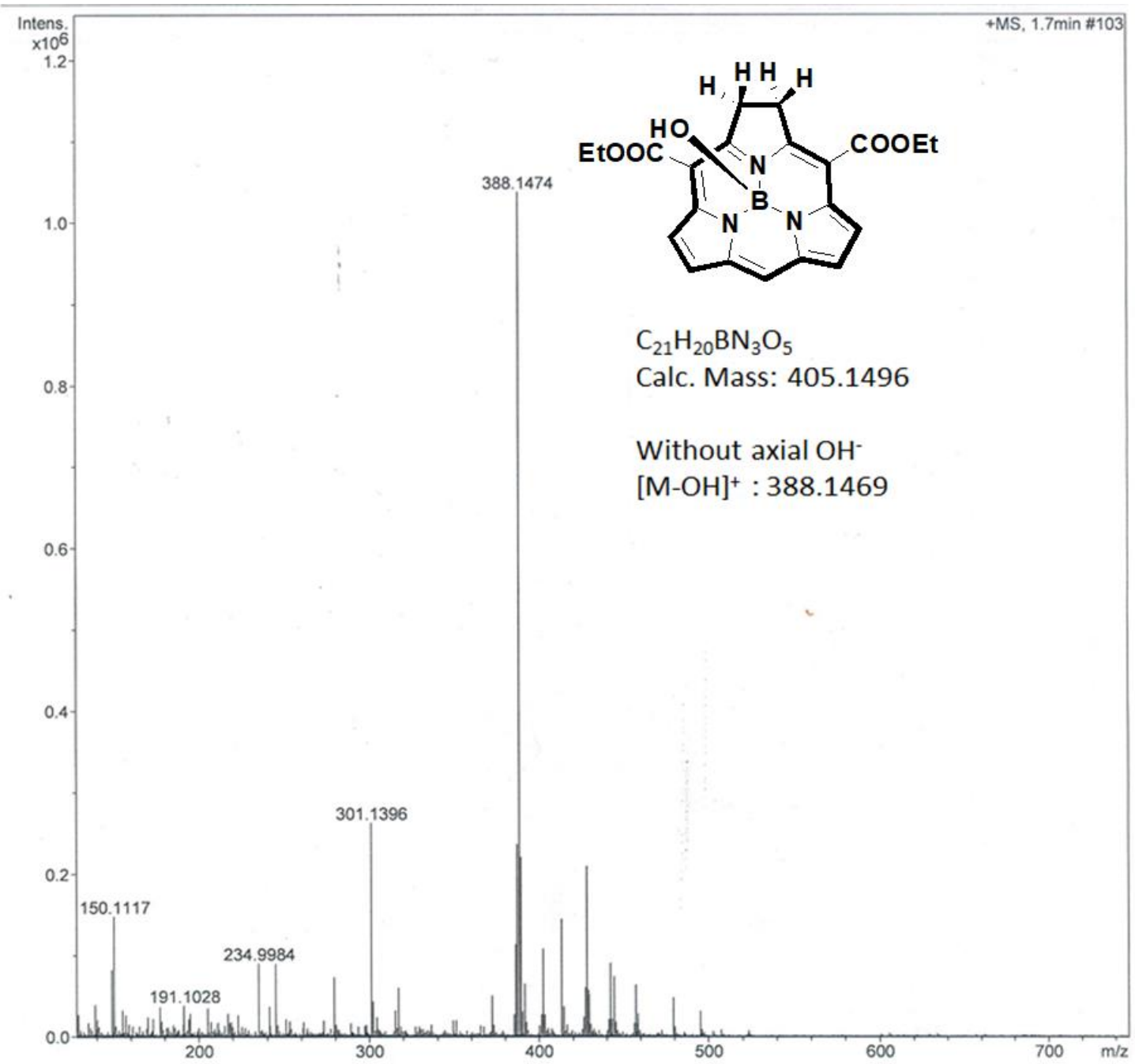

Figure S19: HRMS data of 1a. 

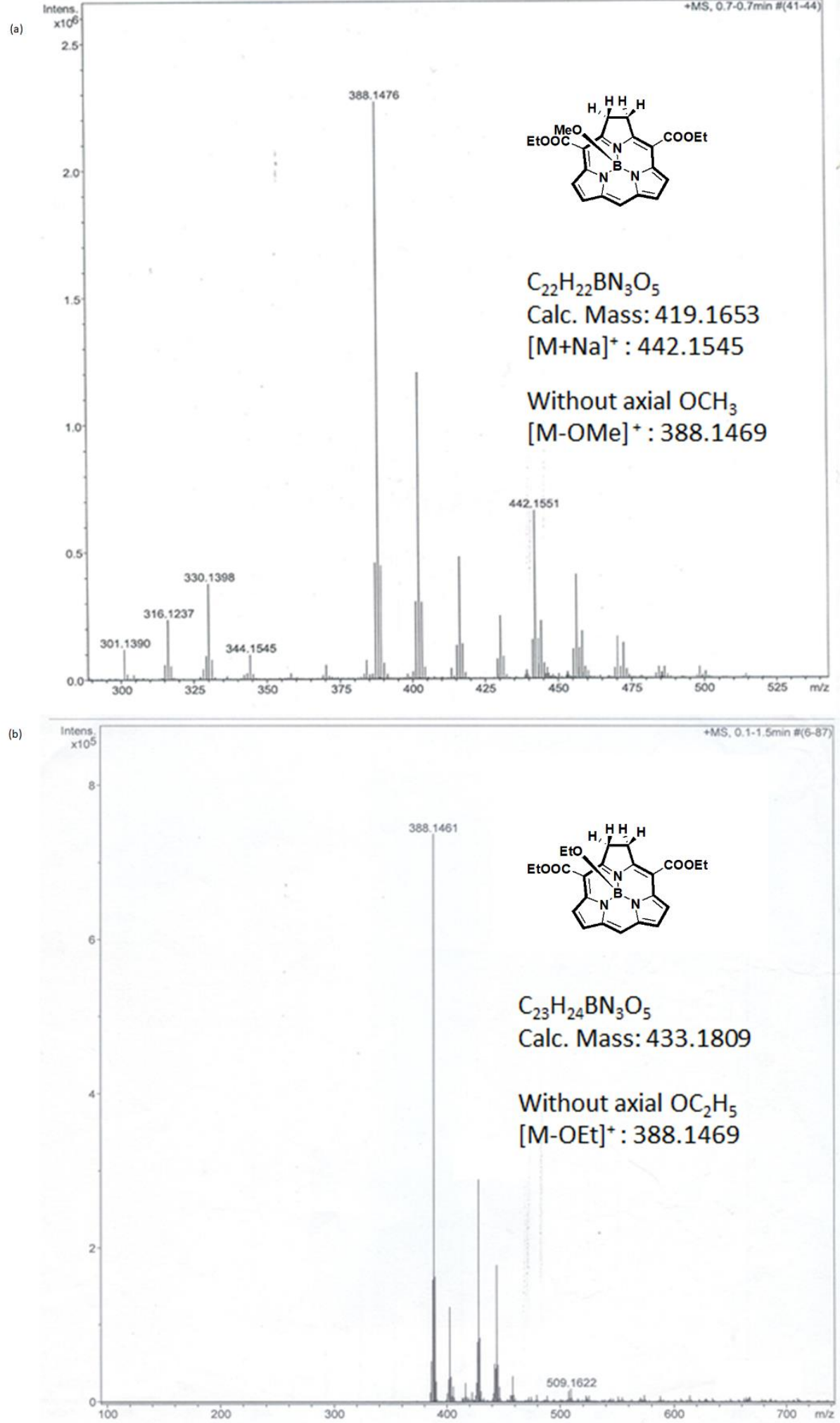

Figure S20: HRMS data of (a) 1b and (b) 1c. 


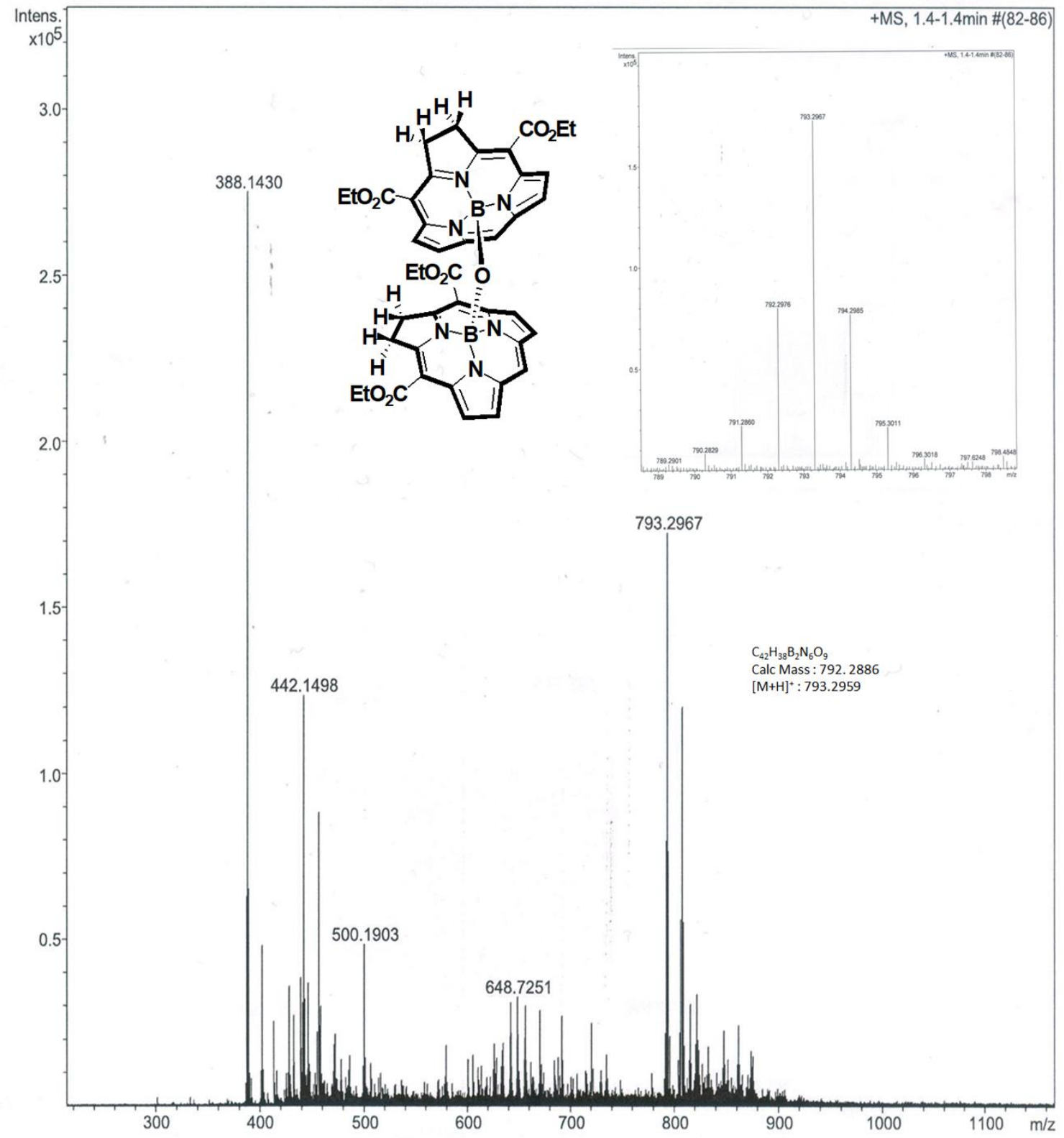

Figure S21: HRMS data of 2. 


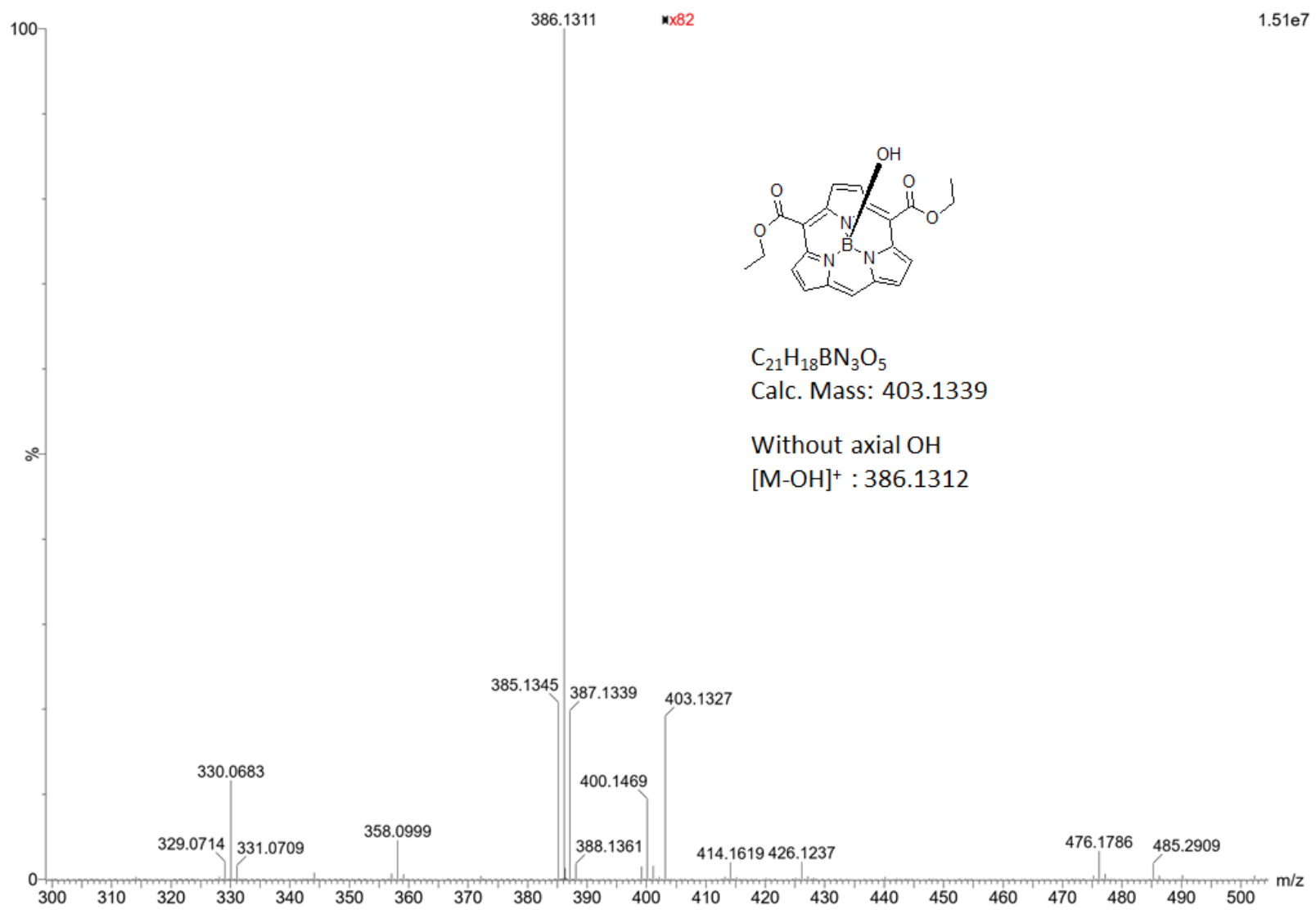

Figure S22: HRMS data of 3. 


\section{SINGLE CRYSTAL XRD ANALYSIS}

\section{5.a. Subchlorin Monomer 1a}

$\mathrm{X}$-ray quality single crystals were obtained by slow diffusion of hexane into chloroform solution of 1a. The crystal was diffracted in $\mathrm{Cu}$ source. The structures were resolved in the monoclinic crystal system with space group $\mathrm{C} 2 / \mathrm{c}$. The asymmetric unit consists of a single monomer subchlorin unit with a slight disorder in ester ethyl group.

Table S1: List of short contacts in 1a

\begin{tabular}{|c|c|c|c|c|}
\hline S1 No. & Interaction & Symm. Opp & $\begin{array}{l}\text { Bond } \\
\text { length }(\AA)\end{array}$ & $\begin{array}{l}\text { Bond length - } \\
\operatorname{VdW}^{*}(\AA)\end{array}$ \\
\hline 1 & $\mathrm{~B}-\mathrm{OH}{ }^{\cdots} \mathrm{O}_{\text {Ester }}$ & 1/2-x,1.5-y,1-z & 2.559 & -0.161 \\
\hline 2 & $\mathrm{~B}-\mathrm{OH}{ }^{\cdots} \mathrm{H}-\mathrm{C}_{\text {meso }}$ & $\mathrm{x},-1+\mathrm{y}, \mathrm{z}$ & 2.628 & -0.092 \\
\hline 3 & $\operatorname{Ester}(\mathrm{H} 2 \mathrm{C}) \mathrm{H}{ }^{\cdots} \mathrm{C}_{\text {Ester }}(\mathrm{C}=\mathrm{O})$ & $1 / 2-\mathrm{x},-1 / 2+\mathrm{y}, 1 / 2-\mathrm{z}$ & 2.842 & -0.058 \\
\hline 4 & $\begin{array}{l}\text { Pyrrolidine }(\mathrm{CH} 2) \mathrm{H}^{\cdots}{ }^{\prime} \pi \text { Pyrrole } \\
(\beta \mathrm{C})\end{array}$ & $\mathrm{x},-1+\mathrm{y}, \mathrm{z}$ & 2.891 & -0.009 \\
\hline 5 & $\operatorname{Ester}(\mathrm{C}=\mathrm{O}) \mathrm{O} \cdots \pi_{\text {Pyrrole }(\alpha \mathrm{C})}$ & $1 / 2-\mathrm{x},-1 / 2+\mathrm{y}, 1 / 2-\mathrm{z}$ & 3.156 & -0.064 \\
\hline 6 & Pyrrole $(\alpha C) \pi^{\cdots} \pi$ Pyrrole ( $\left.\beta C\right)$ & $\mathrm{x},-1+\mathrm{y}, \mathrm{z}$ & 3.385 & -0.015 \\
\hline
\end{tabular}

$* \mathrm{VdW}=$ van der $\mathrm{Waals}$ radius

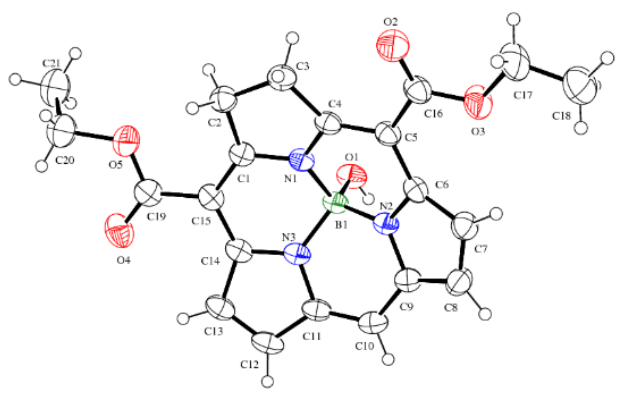

Figure S23: Subchlorin monomer 1a ORTEP diagram (ellipsoid probability 40\%). 


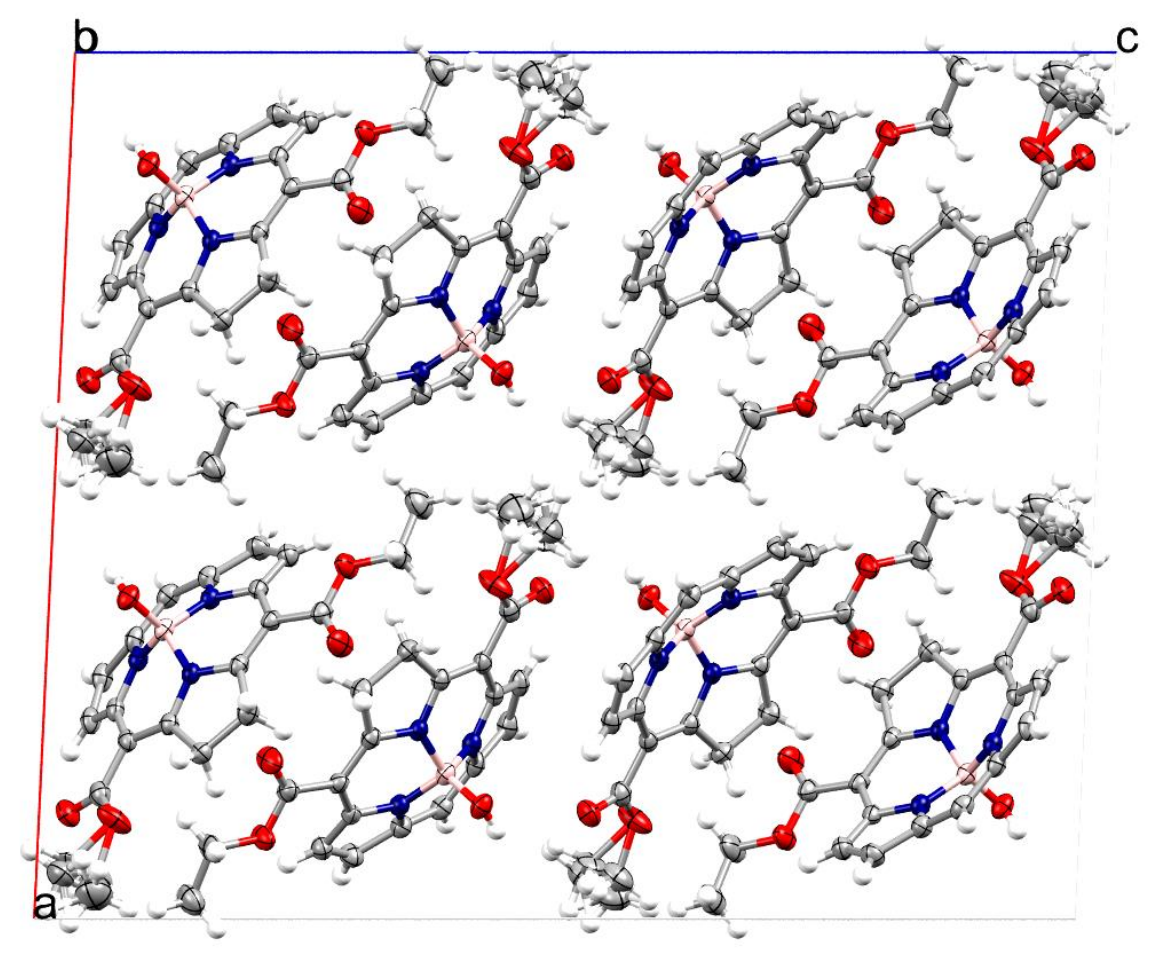

Figure S24: Subchlorin monomer 1a packing diagram (viewed through b axis).

\section{5.b. Subchlorin $\mu$-oxo dimer 2}

X-ray quality single crystals were obtained by slow vapour diffusion of methanol into chloroform solution of 2. The crystal data collection was performed in Mo micro focus source in low temperature. The structure was resolved in the monoclinic crystal system with space group P2 $1 / \mathrm{c}$. Asymmetric unit consists of two independent subchlorin dimer units. The ester ethyl group present in the meso position was found to be highly disordered.

Table S2: List of short contacts in 2

\begin{tabular}{|l|l|l|l|l|}
\hline S1 No. & Interaction & Symm. Opp & $\begin{array}{l}\text { Bond } \\
\text { length }(\AA)\end{array}$ & $\begin{array}{l}\text { Bond length - } \\
\mathrm{VdW}(\AA)\end{array}$ \\
\hline 1 & Pyrrole $(\beta \mathrm{C}) \mathrm{C}-\mathrm{H} \cdots \mathrm{H}-\mathrm{C}_{(\beta \mathrm{C})}$ & $-\mathrm{x},-\mathrm{y}, 1-\mathrm{z}$ & 2.117 & -0.283 \\
\hline 2 & EsterEt.(CH2) $\mathrm{H} \cdots \mathrm{O}=\mathrm{C}_{\text {Ester }}$ & $1-\mathrm{x},-\mathrm{y}, 1-\mathrm{z}$ & 2.462 & -0.258 \\
\hline 3 & Pyrrolidine $(\alpha) \pi \cdots \cdot \mathrm{H}-\mathrm{C}_{\text {Pyrrole }}(\beta)$ & $1-\mathrm{x}, 1 / 2+\mathrm{y}, 1 / 2-\mathrm{z}$ & 2.674 & -0.226 \\
\hline 4 & Pyrrole $(\beta \mathrm{C}) \mathrm{C} \cdots \pi$ Pyrrole $(\beta \mathrm{C})$ & $-\mathrm{x},-\mathrm{y}, 1-\mathrm{z}$ & 2.68 & -0.22 \\
\hline 5 & Ester $\mathrm{C}=\mathrm{O} \cdots \mathrm{H}_{\text {Ester }(\mathrm{CH} 2)}$ & $\mathrm{x}, 1 / 2-\mathrm{y},-1 / 2+\mathrm{z}$ & 2.505 & -0.215 \\
\hline
\end{tabular}




\begin{tabular}{|c|c|c|c|c|}
\hline 6 & 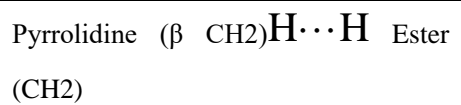 & $-\mathrm{x}, 1 / 2+\mathrm{y}, 1 / 2-\mathrm{z}$ & 2.199 & -0.201 \\
\hline 7 & $\begin{array}{lll}\text { Pyrrolidine } & (\beta & \mathrm{CH} 2) \mathrm{H} \cdots \\
\pi_{\text {Pyrrole }(\beta C)} & & \end{array}$ & $1+\mathrm{x}, \mathrm{y}, \mathrm{z}$ & 2.732 & -0.168 \\
\hline 8 & $\begin{array}{l}\text { Pyrrolidine } \\
\mathrm{O}=\mathrm{C}_{\text {Ester }}\end{array} \quad(\beta \quad \mathrm{CH} 2) \mathrm{H} \cdots$ & $\mathrm{x}, \mathrm{y}, \mathrm{z}$ & 2.566 & -0.154 \\
\hline 9 & Pyrrole $(\beta C) \pi \cdots \pi$ Pyrrole $(\alpha C)$ & $1-\mathrm{x}, 1 / 2+\mathrm{y}, 1 / 2-\mathrm{z}$ & 3.271 & -0.129 \\
\hline 10 & Pyrrole $(\beta C) \mathrm{C}-\mathrm{H} \cdots \mathrm{O}-\mathrm{B}_{\text {axial }}$ & $1-\mathrm{x}, 1 / 2+\mathrm{y}, 1 / 2-\mathrm{z}$ & 2.592 & -0.128 \\
\hline 11 & Pyrrole $(\beta C) \pi \cdots \pi$ Pyrrole $(\beta C)$ & $1-\mathrm{x}, 1 / 2+\mathrm{y}, 1 / 2-\mathrm{z}$ & 3.276 & -0.124 \\
\hline 12 & $\begin{array}{llll}\text { Pyrrolidine } & (\beta & \mathrm{CH} 2) \mathrm{H} \cdots & \pi \\
\text { Pyrrole }(\alpha \mathrm{C}) & & & \\
\end{array}$ & $1+\mathrm{x}, \mathrm{y}, \mathrm{z}$ & 2.781 & -0.119 \\
\hline 13 & Ester $\mathrm{C}=\mathrm{O} \cdots \mathrm{H}$ Pyrrolidine $(\beta \mathrm{CH} 2)$ & $1+\mathrm{x}, \mathrm{y}, \mathrm{z}$ & 2.605 & -0.115 \\
\hline
\end{tabular}

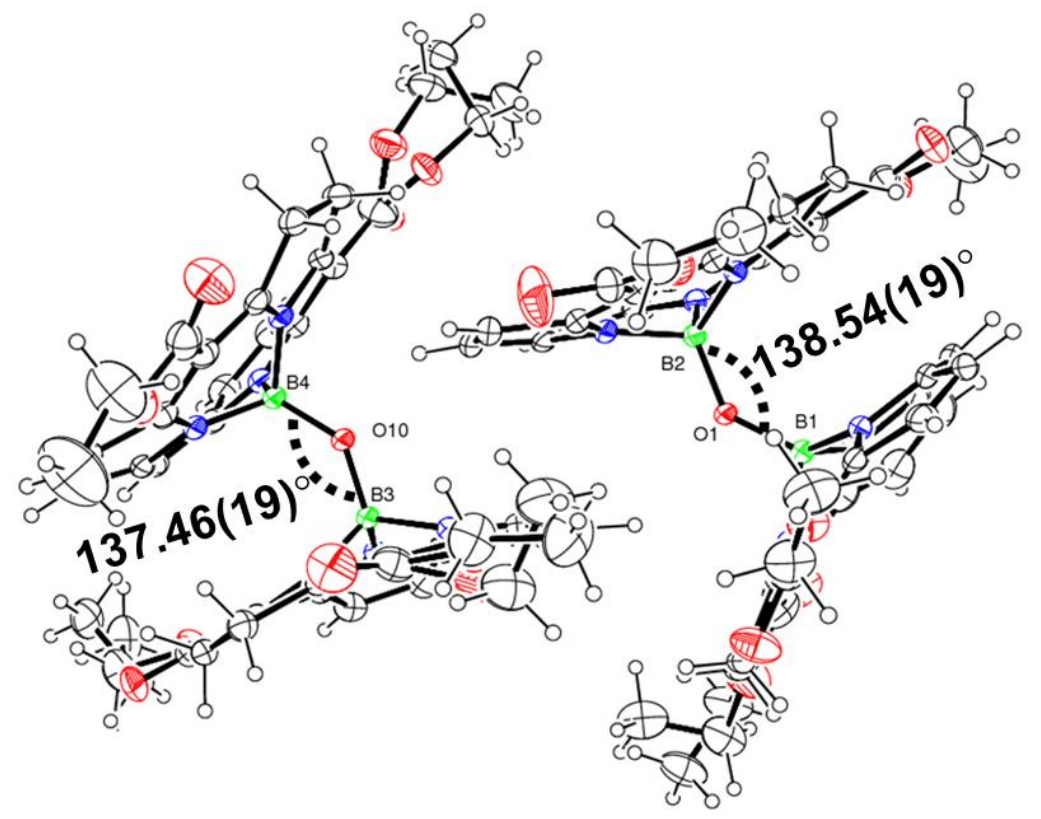

Figure S25: Subchlorin dimer 2 ORTEP diagram (two molecules in the assymetic unit), where the disordered ethyl groups were removed for clarity (ellipsoid probability $40 \%$ ). 


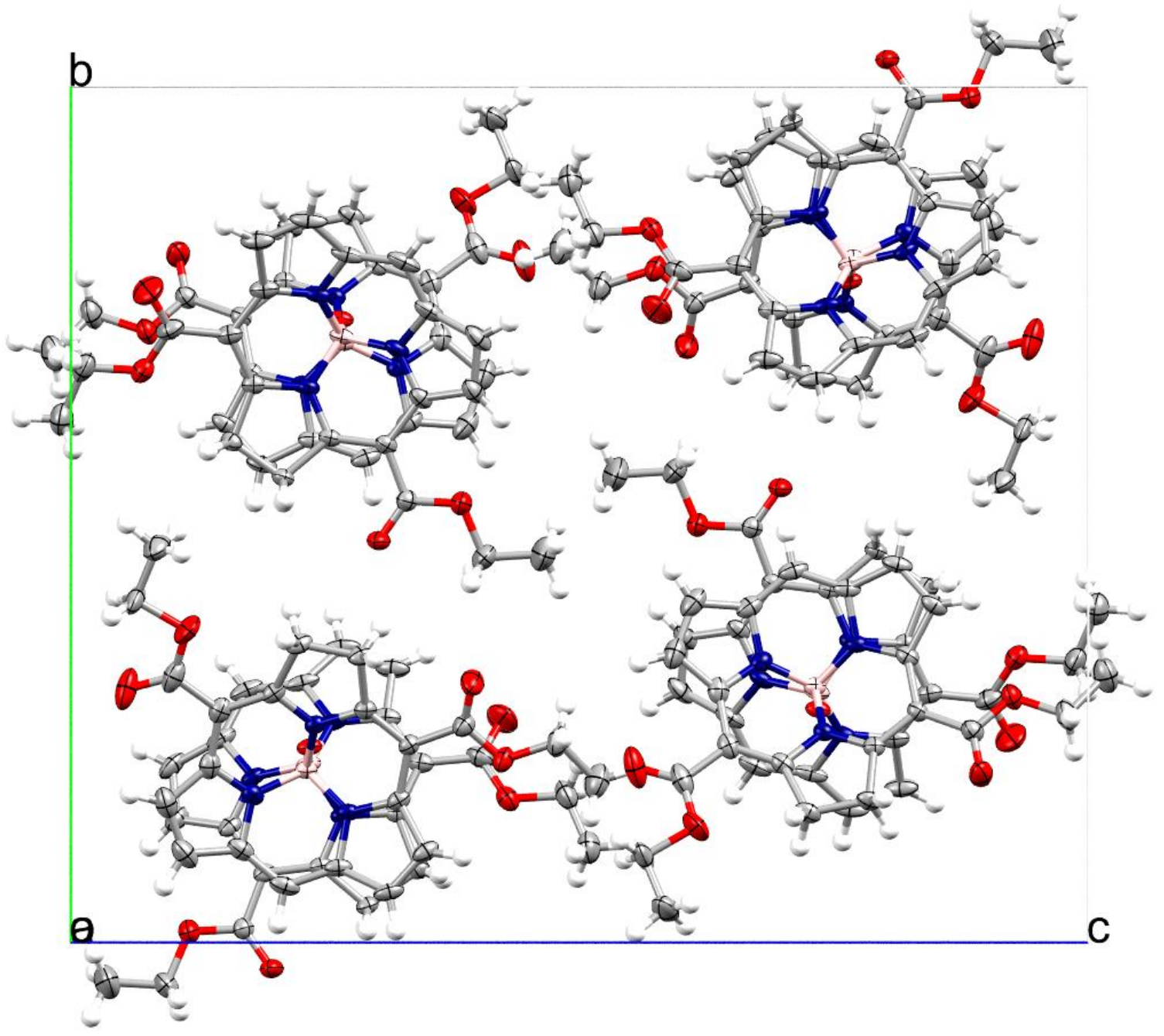

Figure S26: Subchlorin dimer 2 selected molecules top on view in packing diagram (viewed through 'a' axis, disordered groups removed for clarity). 
(a)
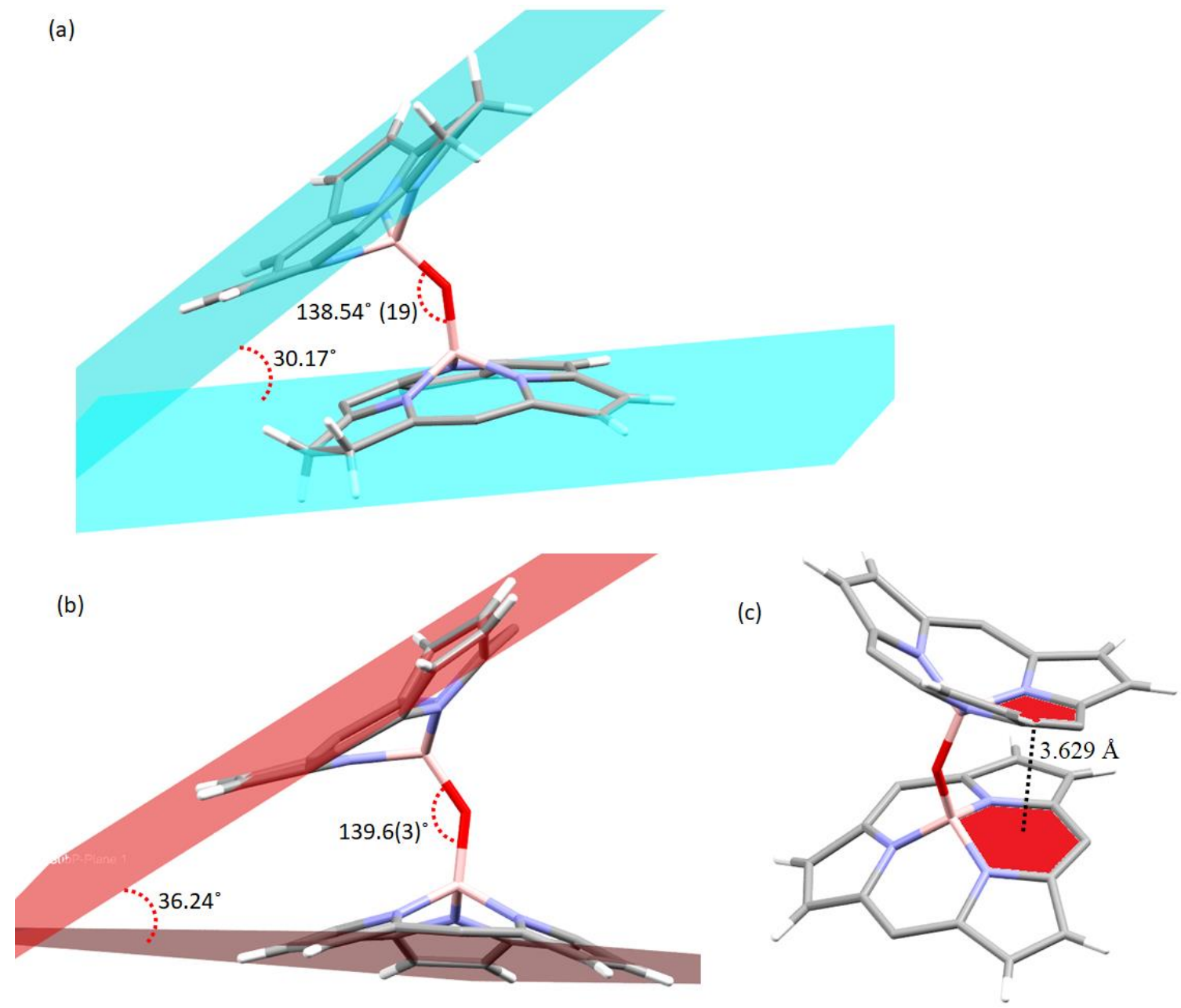

Figure S27: Dihedral angle between two chromophore planes of dimers (a) 2 (b) 5. Planes were drawn by considering each subchlorin core (c) calculated distance between centroids of closest sixmembered rings in 5 (meso- ester and phenyl groups were omitted for clarity).

\section{5.c. Subporphyrin 3}

Small X-ray quality single crystals were obtained by slow evaporation of chloroform solution of subporphyrin 3. The crystal data collection was performed in Mo micro focus source in room temperature. The structure was resolved in the monoclinic crystal system with space group P2 $1 / \mathrm{c}$. Asymmetric unit consists of a subporphyrin unit and a chloroform molecule. 


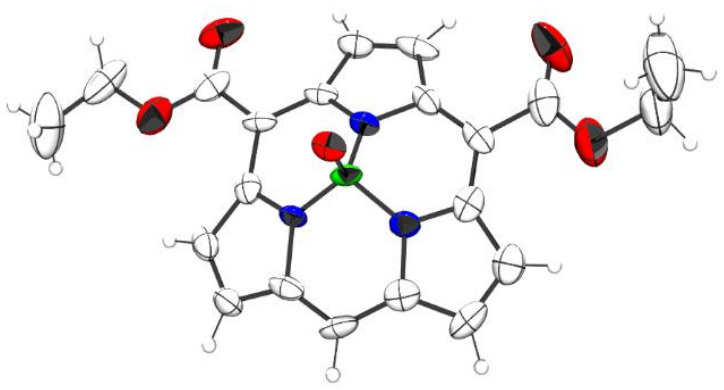

Figure S28: ORTEP diagram of $\mathbf{3}$ (solvent molecule is removed for clarity). Ellipsoid are drawn with $40 \%$ probability.

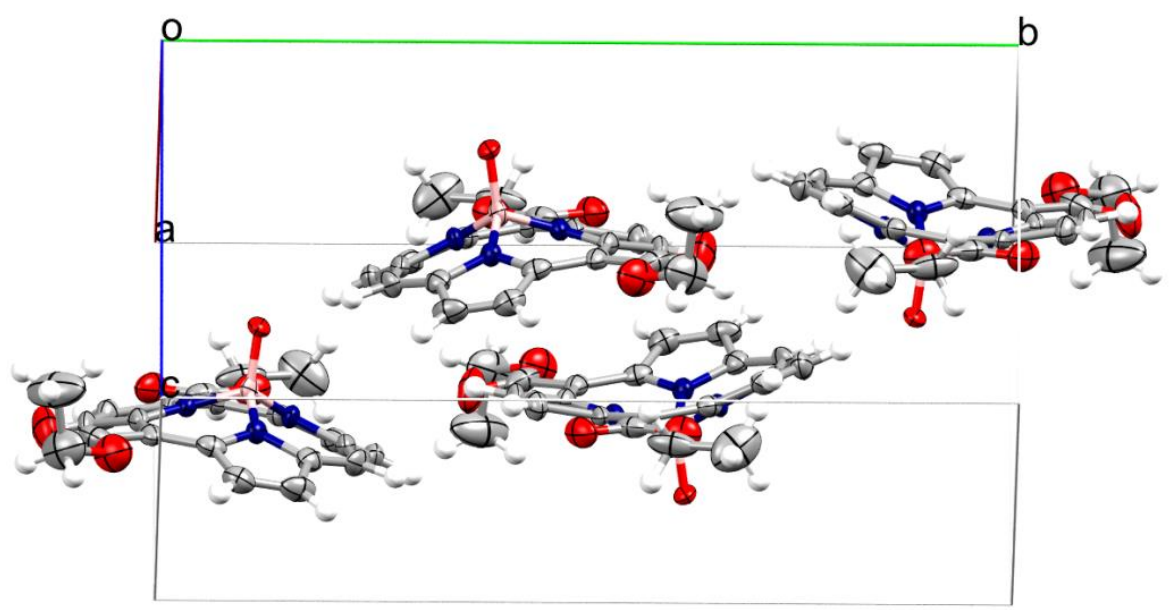

Figure S29: Subporphyrin 3 packing diagram (four molecules in unit cell, solvent molecule is removed for clarity).

Table S3: Crystal data table for $\mathbf{1 a}, \mathbf{2}$ and $\mathbf{3}$.

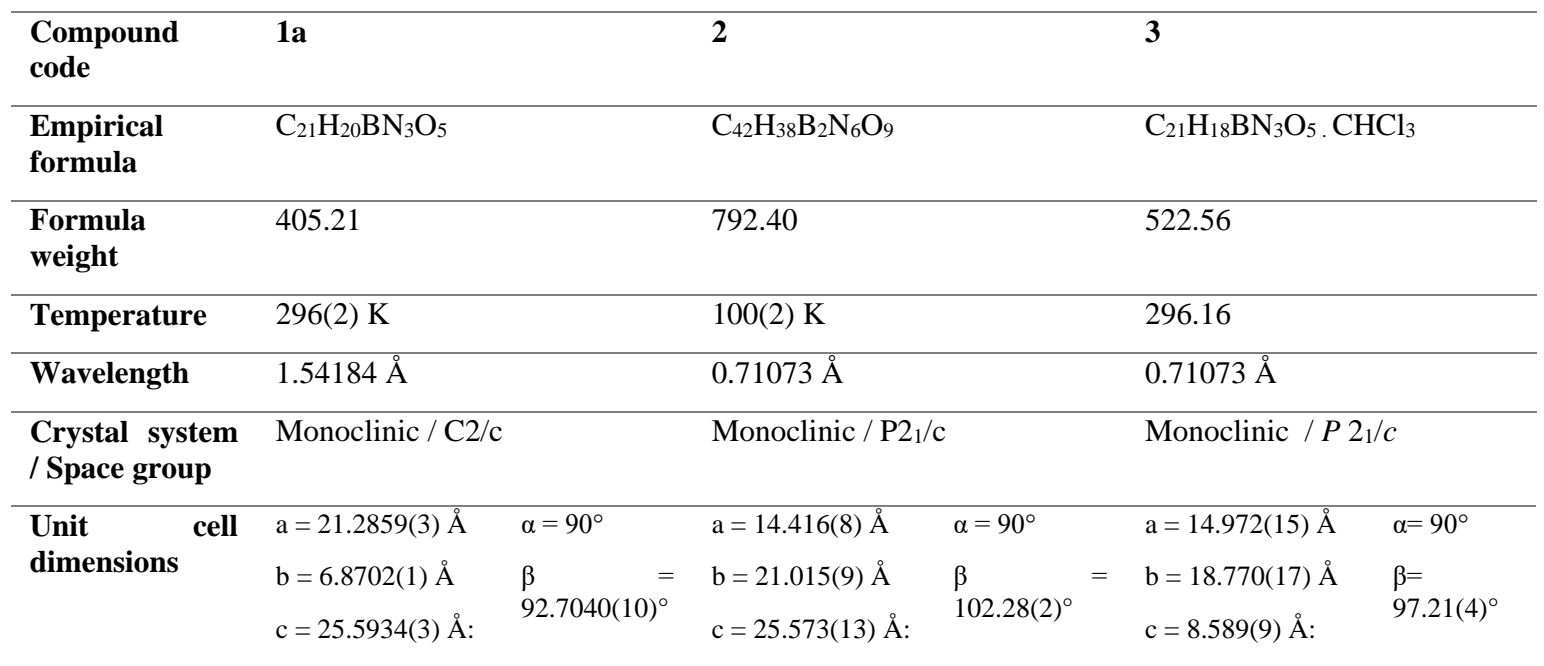




\begin{tabular}{|c|c|c|c|}
\hline & $\gamma=90^{\circ}$ & $\gamma=90^{\circ}$ & $\gamma=90^{\circ}$ \\
\hline & $V: 3738.57(9) \AA^{3}$ & $V: 7571.0(7) \AA^{3}$ & $V: 2418(4) \AA^{3}$ \\
\hline $\mathbf{Z}$ & 8 & 8 & 4 \\
\hline Density (calc.) & $1.440 \mathrm{Mg} / \mathrm{m}^{3}$ & $1.390 \mathrm{Mg} / \mathrm{m}^{3}$ & $1.449 \mathrm{Mg} / \mathrm{m}^{3}$ \\
\hline $\begin{array}{l}\text { Absorption } \\
\text { coefficient }\end{array}$ & $0.852 \mathrm{~mm}^{-1}$ & $0.098 \mathrm{~mm}^{-1}$ & $0.422 \mathrm{~mm}^{-1}$ \\
\hline $\begin{array}{l}\theta \text { range (data } \\
\text { collection) }\end{array}$ & 3.458 to $69.313^{\circ}$ & 2.398 to $23.341^{\circ}$ & 2.567 to $24.249^{\circ}$ \\
\hline Index ranges & $\begin{array}{l}-25<=\mathrm{h}<=25, \quad-8<=\mathrm{k}<=8, \\
31<=\mathrm{l}<=31\end{array}$ & $\begin{array}{l}-15<=\mathrm{h}<=16, \quad-23<=\mathrm{k}<=23, \quad- \\
28<=1<=28\end{array}$ & $\begin{array}{l}-17<=\mathrm{h}<=16,-21<=\mathrm{k}<=21,- \\
9<=\mathrm{l}<=9\end{array}$ \\
\hline $\begin{array}{l}\text { Reflections coll. } \\
\text { / Independent } \\
\text { reflect. }\end{array}$ & $24094 / 3436[\mathrm{R}(\mathrm{int})=0.1167]$ & $63088 / 10942[\mathrm{R}(\mathrm{int})=0.0523]$ & $19177 / 3856\left[\mathrm{R}_{\mathrm{int}}=0.1391\right]$ \\
\hline $\begin{array}{l}\text { Completeness } \\
\text { to } \Theta=67.684^{\circ}\end{array}$ & $99.9 \%$ & $99.5 \%$ & $99.8 \%$ \\
\hline $\begin{array}{l}\text { Absorption } \\
\text { correction }\end{array}$ & $\begin{array}{l}\text { Semi-empirical } \\
\text { equivalents }\end{array}$ & $\begin{array}{l}\text { Semi-empirical } \\
\text { equivalents }\end{array}$ & $\begin{array}{l}\text { Semi-empirical } \\
\text { equivalents }\end{array}$ \\
\hline $\begin{array}{l}\text { Max. and min. } \\
\text { transmission }\end{array}$ & 0.7531 and 0.6510 & 0.91 and 0.87 & 0.7452 and 0.6528 \\
\hline $\begin{array}{l}\text { Refinement } \\
\text { method }\end{array}$ & Full-matrix least-squares on F2 & Full-matrix least-squares on F2 & $\begin{array}{l}\text { Full-matrix least-squares on } \\
\text { F2 }\end{array}$ \\
\hline $\begin{array}{ll}\text { Data } & / \\
\text { restraints } & / \\
\text { parameters } & \end{array}$ & $3436 / 40 / 292$ & 10942 / 239 / 1170 & $3856 / 1 / 313$ \\
\hline $\begin{array}{l}\text { Goodness-of-fit } \\
\text { on } F^{2}\end{array}$ & 1.022 & 1.030 & 1.048 \\
\hline $\begin{array}{l}\text { Final } \mathbf{R} \text { indices } \\
{[\mathbf{I}>2 \operatorname{sigma}(\mathbf{I})]}\end{array}$ & $\mathrm{R} 1=0.0601, \mathrm{wR} 2=0.1186$ & $\mathrm{R} 1=0.0509, \mathrm{wR} 2=0.1285$ & $\mathrm{R} 1=0.0986, \mathrm{wR} 2=0.2699$ \\
\hline $\begin{array}{l}\mathbf{R} \text { indices (all } \\
\text { data) }\end{array}$ & $\mathrm{R} 1=0.1106, \mathrm{wR} 2=0.1383$ & $\mathrm{R} 1=0.0678, \mathrm{wR} 2=0.1407$ & $\mathrm{R} 1=0.1680, \mathrm{wR} 2=0.3190$ \\
\hline $\begin{array}{l}\text { Largest diff. } \\
\text { peak and hole }\end{array}$ & 0.202 and -0.214 e. $\stackrel{\AA}{A}-3$ & 0.516 and -0.310 e..$\AA^{-3}$ & 0.642 and -0.605 e. $\AA^{-3}$ \\
\hline
\end{tabular}




\section{6. a. Absorption and emission studies}
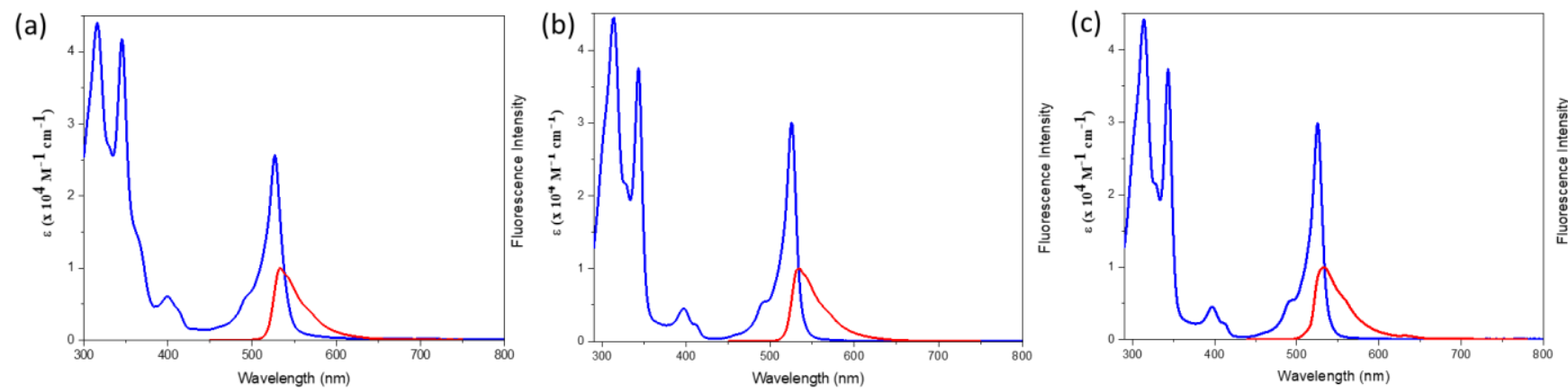

Figure S30: Absorption (blue) and emission (red) spectra of $\mathbf{1}$ [1a (a), $\mathbf{1 b}$ (b) and 1c (c)] in $\mathrm{CH}_{2} \mathrm{Cl}_{2}$.

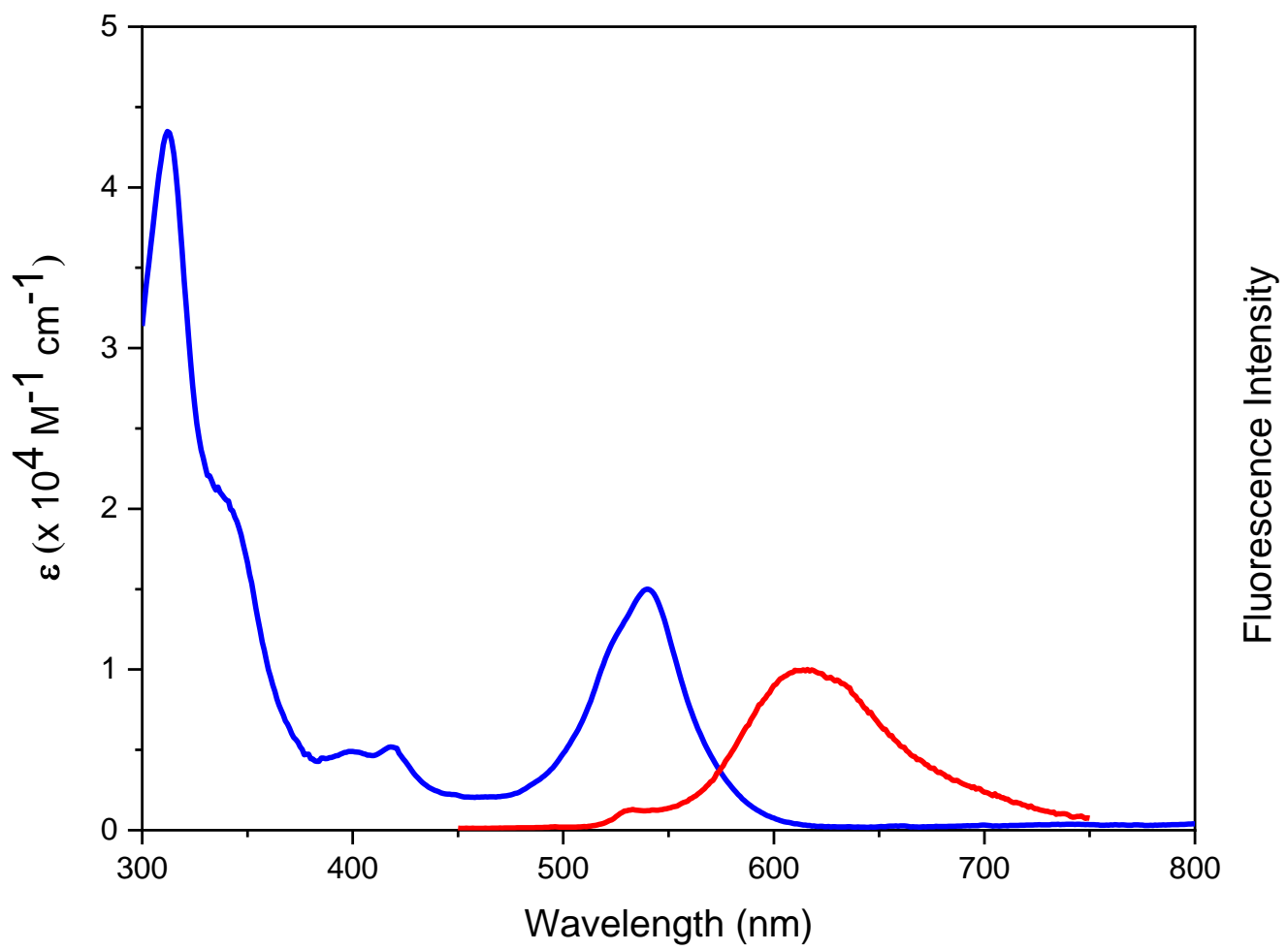

Figure S31: Absorption (blue) and emission (red) spectra of 2 in $\mathrm{CH}_{2} \mathrm{Cl}_{2}$. 


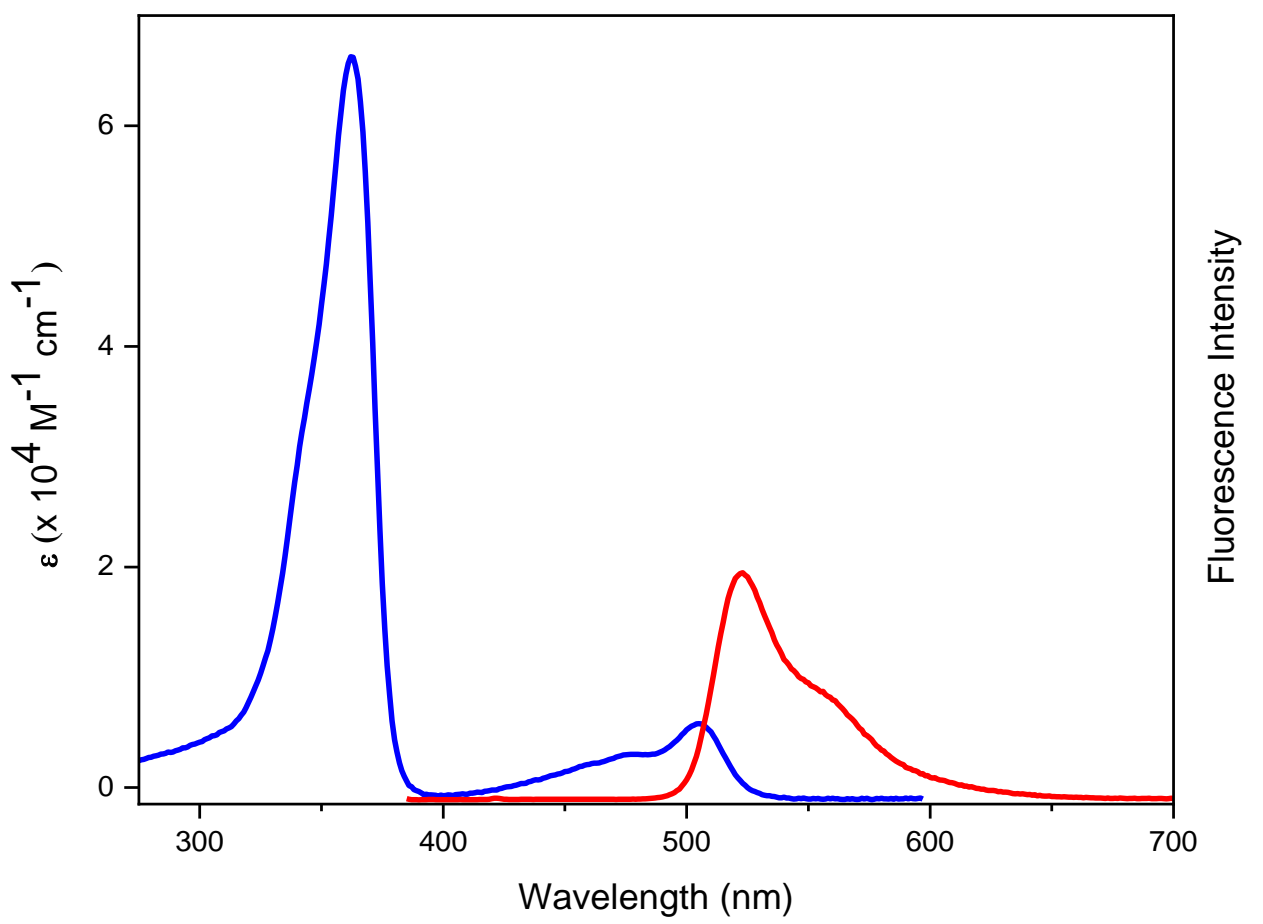

Figure S32: Absorption (blue) and emission (red) spectra of 3 in $\mathrm{CH}_{2} \mathrm{Cl}_{2}$. 


\section{6. b. Subchlorin solvatochromic studies}

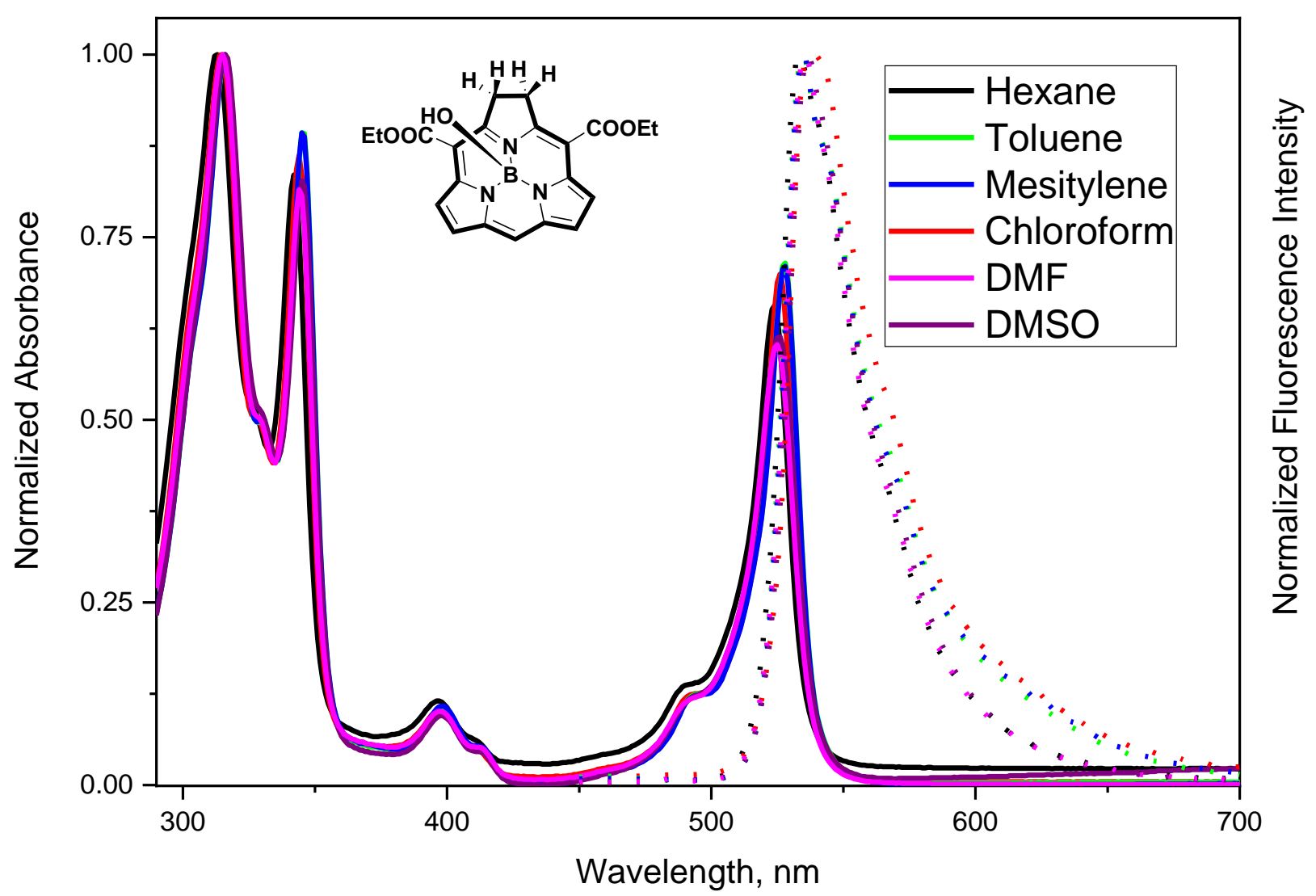

Figure S33: Absorption (solid lines) and emission spectra (doted lines) of subchlorin 1a in different solvents.

Table S4: Solvatochromic studies: Monomer 1a Absorbance and emission $\lambda_{\max }$ Values (nm) in different solvents.

\begin{tabular}{|l|l|l|l|l|l|l|l|l|l|l|l|}
\hline \multicolumn{2}{|l|}{ Monomer 1a Absorbance and emission $\lambda \max$ Values $(\mathrm{nm})$} \\
\hline Chloroform & Toluene & Mesitylene & \multicolumn{1}{l|}{ DMF } & \multicolumn{2}{l|}{ DMSO } & \multicolumn{2}{l|}{ Hexane } \\
\hline Abs & Emi & Abs & Emi & Abs & Emi & Abs & Emi & Abs & Emi & Abs & Emi \\
\hline 315 & & 316 & & 316 & & 315 & & 317 & & 315 & \\
\hline 344 & & 345 & & 345 & & 344 & & 347 & & 344 & \\
\hline 398 & & 398 & & 398 & & 397 & & 401 & & 398 & \\
\hline 526 & 538 & 528 & 534 & 528 & 535 & 525 & 535 & 526 & 538 & 526 & 531 \\
\hline
\end{tabular}




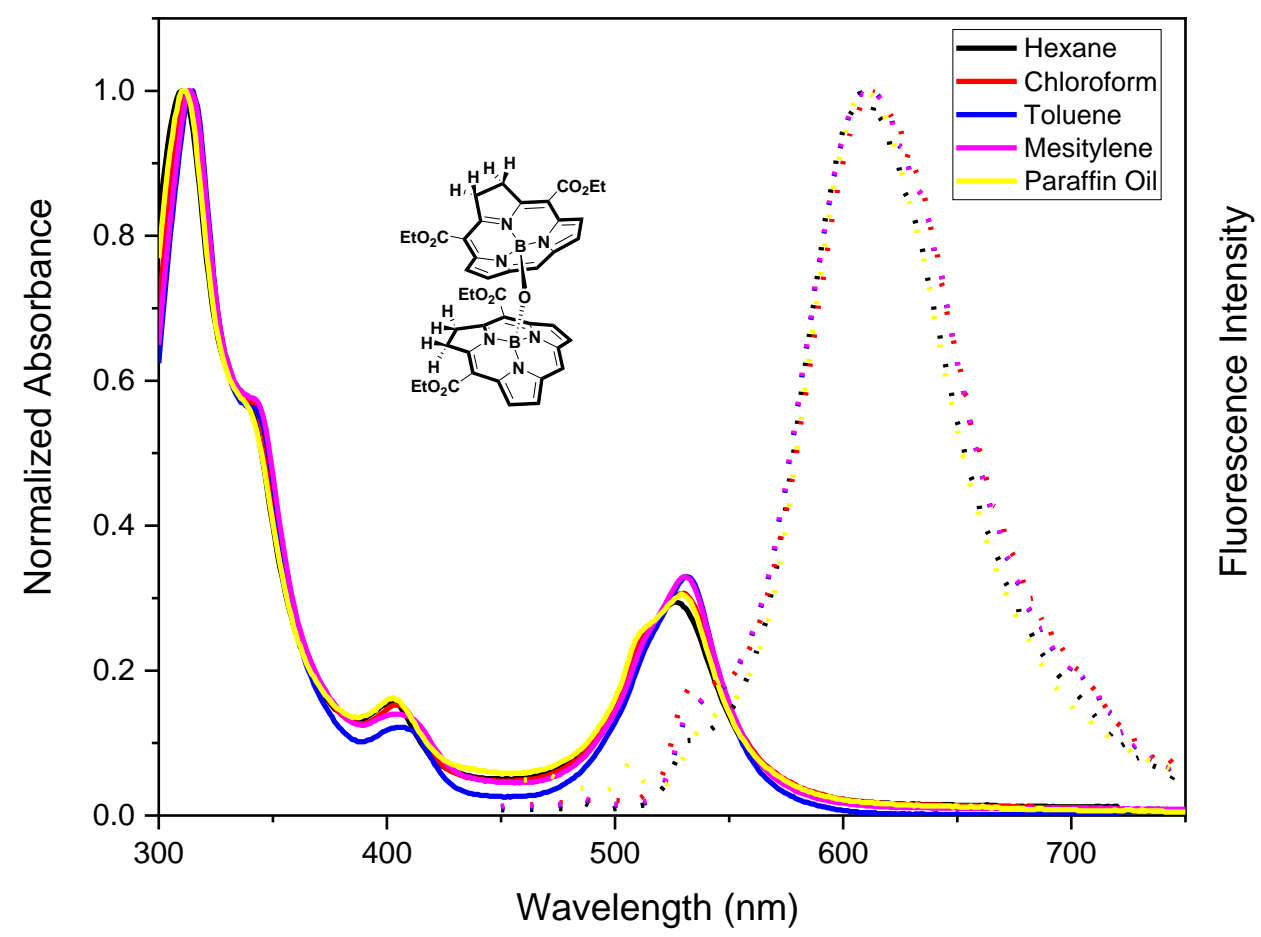

Figure S34: Absorption and emission spectra of subchlorin dimer 2 in different solvents.

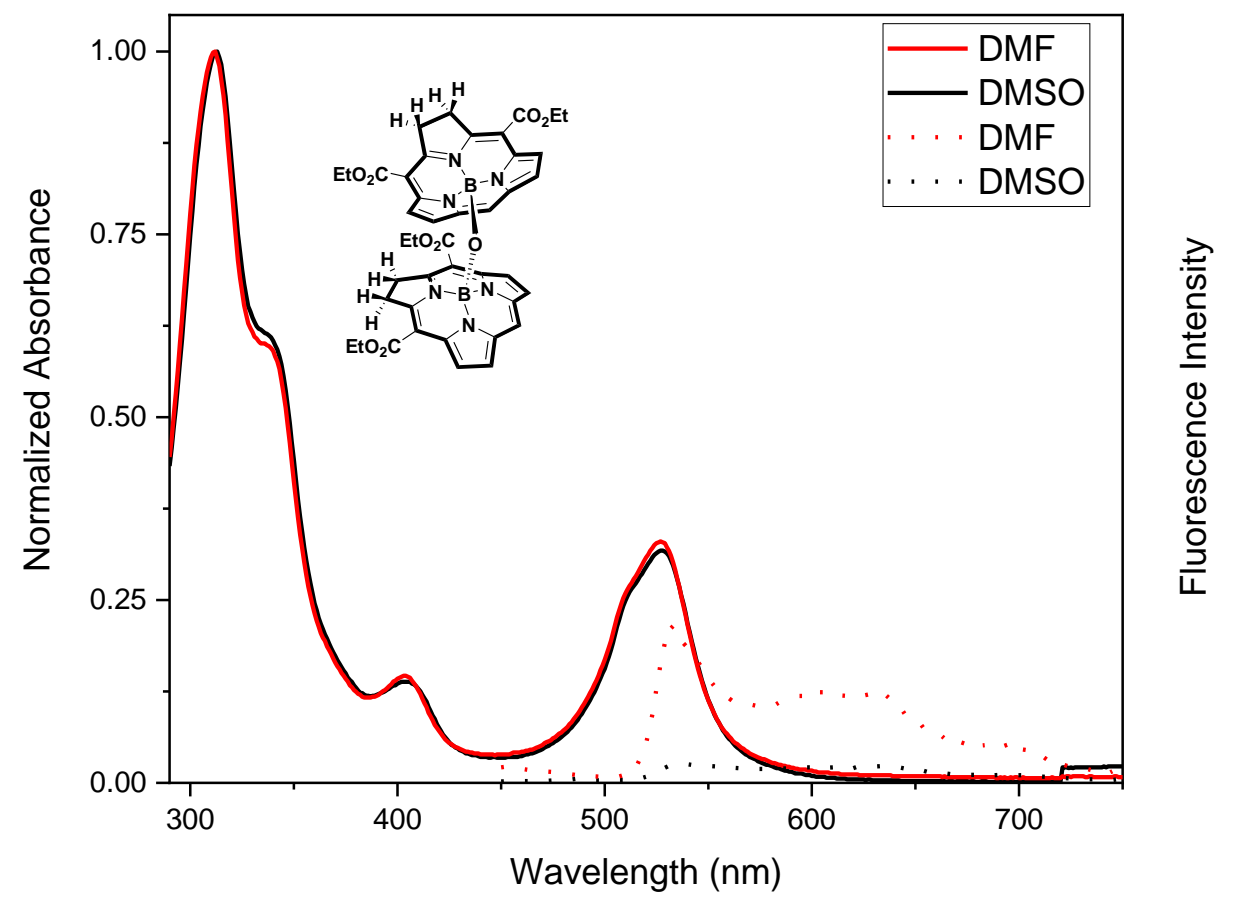

Figure S35: Absorption and emission spectra of subchlorin dimer 2 in DMF and DMSO (as the emission intensities are very poor in these solvents with respect to that in less polar solvents, they are shown differently). 
Table S5: Solvatochromic studies: Dimer 2 Absorbance and emission $\lambda_{\max }$ values $(\mathrm{nm})$ in different solvents.

\begin{tabular}{|l|l|l|l|l|l|l|l|l|l|l|l|l|l|}
\hline \multicolumn{1}{|l|}{ Dimer 2 absorbance and emission $\lambda$ max values (nm) } \\
\hline Chloroform & Toluene & Mesitylene & DMF & \multicolumn{1}{l|}{ DMSO } & \multicolumn{1}{l|}{ Hexane } & \multicolumn{2}{l|}{ Paraffin oil } \\
\hline Abs & Emi & Abs & Emi & Abs & Abs & Abs & Emi & Abs & Emi & Abs & Emi & Abs & Emi \\
\hline 313 & & 315 & & 314 & & 312 & & 313 & & 313 & & 311 & \\
\hline 404 & & 406 & & 404 & & 404 & & 406 & & 404 & & 404 & \\
\hline 530 & 613 & 532 & 610 & 530 & 610 & 527 & 632 & 528 & 545 & 530 & 612 & 532 & 610 \\
\hline
\end{tabular}




\section{FLUORESCENCE LIFE TIME ANALYSIS}
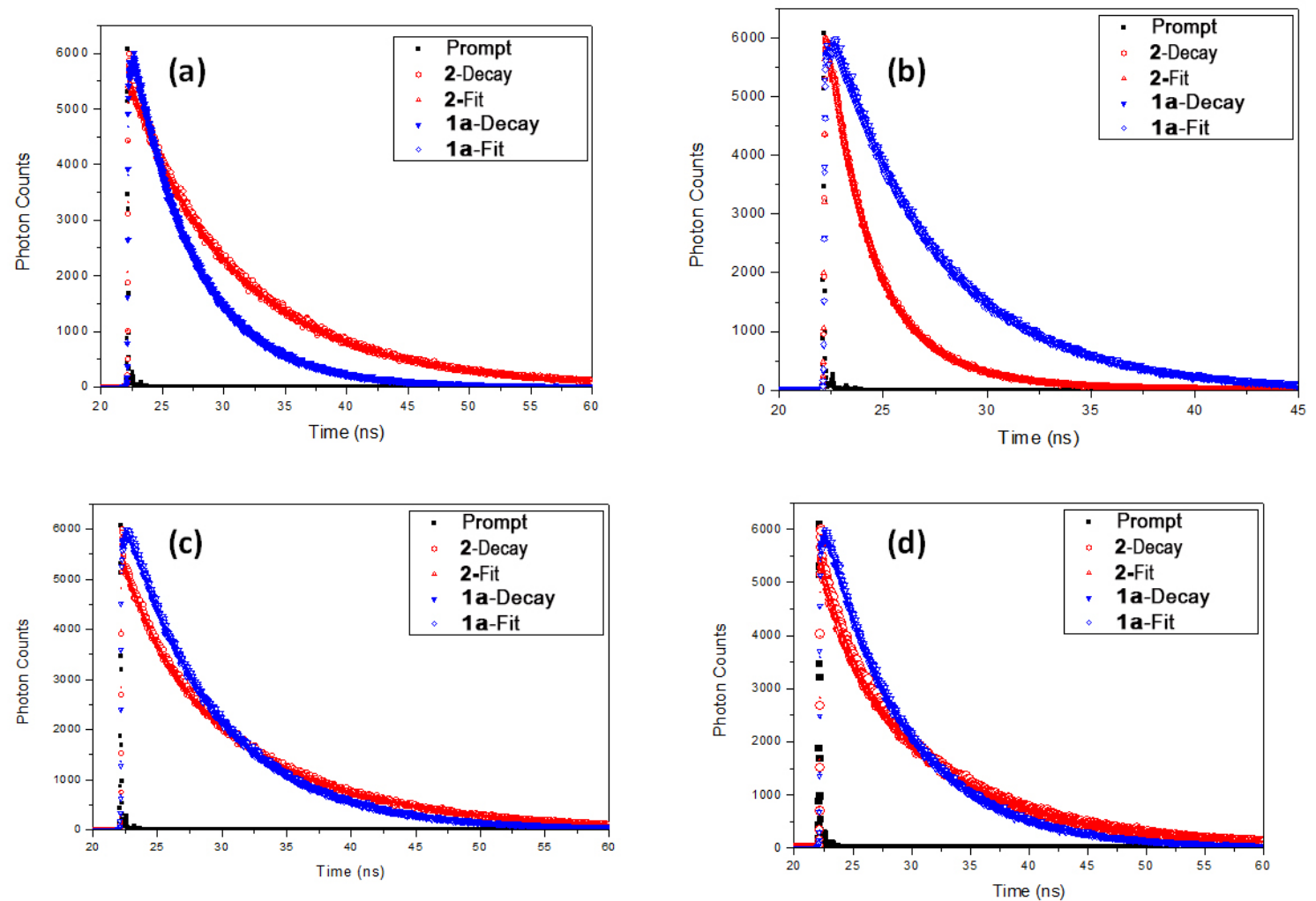

Figure S36: Fluorescence emission decays of subchlorin monomer 1a (blue) and dimer 2 (red) in (a) chloroform, (b) DMF, (c) toluene and (d) mesitylene following excitation at $405 \mathrm{~nm}$. 
Table S6: Quantum yield $\left(\phi_{\mathrm{f}}\right)$ and lifetime values for $\mathbf{1 a}$ and 2.

\begin{tabular}{|l|l|l|l|l|l|l|}
\hline \multirow{2}{*}{ Solvents } & \multicolumn{2}{|c|}{ 1a } & \multicolumn{4}{c|}{ 2 } \\
\cline { 2 - 7 } & \multirow{2}{*}{$\phi_{f}$} & \multirow{2}{*}{$\tau(\mathrm{ns})$} & \multirow{2}{*}{$\Phi_{f}$} & \multicolumn{3}{c|}{$\tau(\mathrm{ns})$} \\
\cline { 5 - 8 } & & & & $\tau_{1}$ & $\tau_{2}$ & $\tau_{\text {avg }}$ \\
\hline Mesitylene & 0.4 & 7.1 & 0.2 & 2.66 & 10.6 & 8.31 \\
\hline Toluene & 0.37 & 7.33 & 0.2 & 3.07 & 10.5 & 8.47 \\
\hline Chloroform & 0.39 & 5.3 & 0.12 & 4.1 & 10.1 & 9.06 \\
\hline DMF & 0.35 & 5.35 & 0.02 & 2.02 & 4.8 & 2.42 \\
\hline DMSO & 0.33 & 5.63 & - & 1.75 & 5.75 & 2 \\
\hline
\end{tabular}




\section{Singlet oxygen Luminescence and Quantum yield analysis}
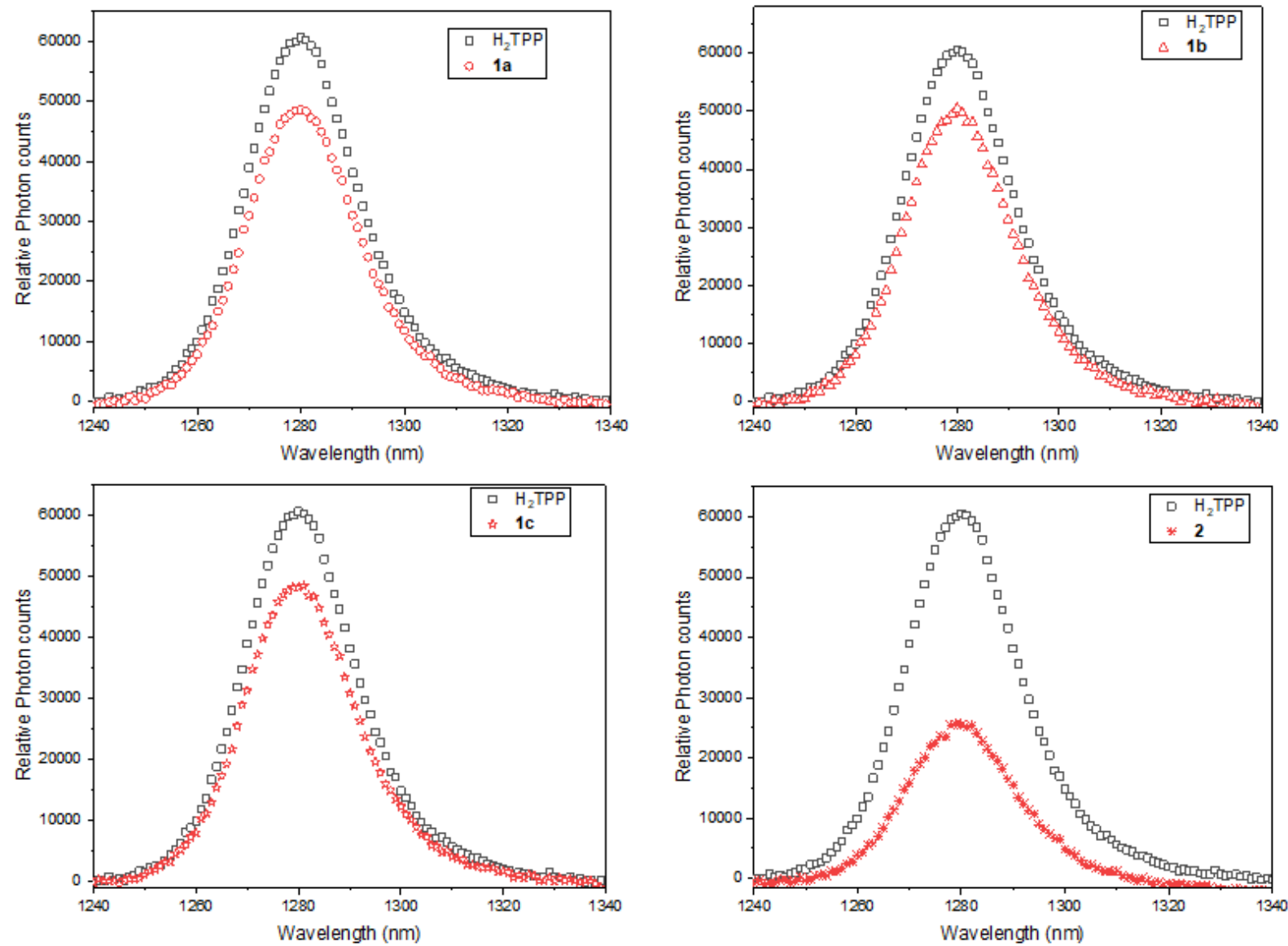

Figure S37: Singlet oxygen luminescence of optically matched $\mathbf{H}_{2} \mathbf{T P P}$ (reference $\phi_{\Delta}$ 0.7); 1a, 1b, $1 \mathrm{c}$ and 2 in air saturated toluene solution at $25^{\circ} \mathrm{C}\left(\lambda_{\mathrm{ex}}=510 \mathrm{~nm}\right)$. [ $\phi_{\Delta}$ calculated by equation S1].

Table S7: Singlet oxygen quantum yield $\left(\phi_{\Delta}\right)$ for $\mathbf{1 b}, \mathbf{1 c}$ and $\mathbf{2}$ in toluene.

\begin{tabular}{|c|c|}
\hline Compound & Singlet oxygen quantum yield $\left(\phi_{\Delta}\right)$ \\
\hline $1 \mathrm{a}$ & 0.56 \\
\hline $1 \mathrm{~b}$ & 0.58 \\
\hline $1 \mathrm{c}$ & 0.56 \\
\hline 2 & 0.30 \\
\hline
\end{tabular}




\section{Cyclic Voltammograms}
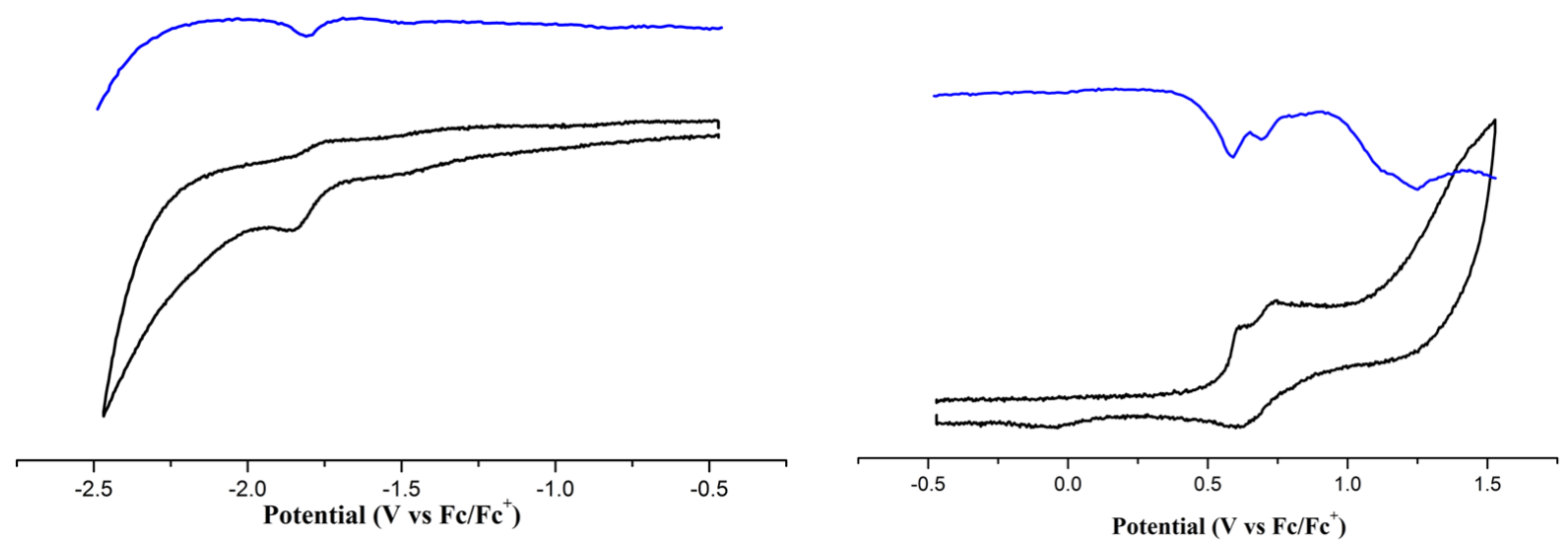

Figure S38: Cyclic Voltammograms of $\mathbf{1 b}$ recorded in DCM with $\mathrm{TBAPF}_{6}$ as supporting electrolyte with scan rate of $50 \mathrm{mV} / \mathrm{sec}$.
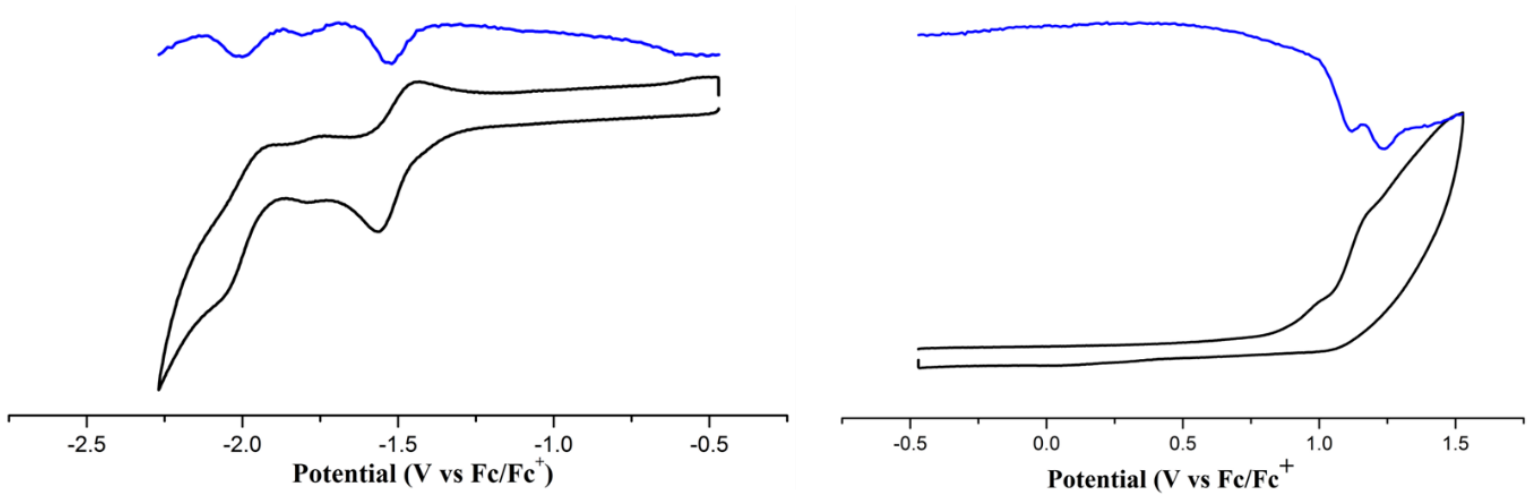

Figure S39: Cyclic Voltammograms of 3 recorded in DCM with TBAPF $_{6}$ as supporting electrolyte with scan rate of $50 \mathrm{mV} / \mathrm{sec}$. 


\section{Computational Analysis: Structure, Energetics, and Spectra}

The geometry optimization and frequency calculations are performed on the molecules at MPW1PW91/6-31G(d,p) level of theory ${ }^{\mathrm{S} 6}$ using Gaussian09 suite of programs. ${ }^{\mathrm{S}}$ The stationary points were characterized by the absence of imaginary vibrational frequencies. The relative energies of the dimer molecules are depicted in Figure S40. The absorption spectra are calculated at the WB97XD/def2-SVP level of theory. The effect of the solvent is included using the solvation model based on density $(\mathrm{SMD})^{\mathrm{S} 8}$ with dichloromethane as the solvent, as implemented in the Gaussian 09 package. The different isomers of dimer 2 were constructed by rotating around the B-O-B bond and considering all possible orientations of the chromophores with respect to each other. The crystal structure coordinates were considered as the starting structures for the geometry optimizations at MPW1PW91/6-31g(d,p), and the absorption spectrum is calculated from the optimized geometries at WB97XD/def2-SVP. All the molecules considered

were with singlet spin multiplicity and neutral charge. The Cartesian coordinates (in $\AA$ ) and the vibrational frequencies (in $\mathrm{cm}^{-1}$ ) of the most stable molecules at MPW1PW91/6-31g(d) are reported in Tables S8-S13. All the 3N-6 vibrational mode frequencies reported in Table S8-S13 are positive, and there are no imaginary frequencies for the optimized structures. We have considered only the most stable monomer and dimer molecule for the absorption spectrum calculation and further comparison with the experiment. The absorption maxima and the intensity of the spectra are reported in Table S7. 

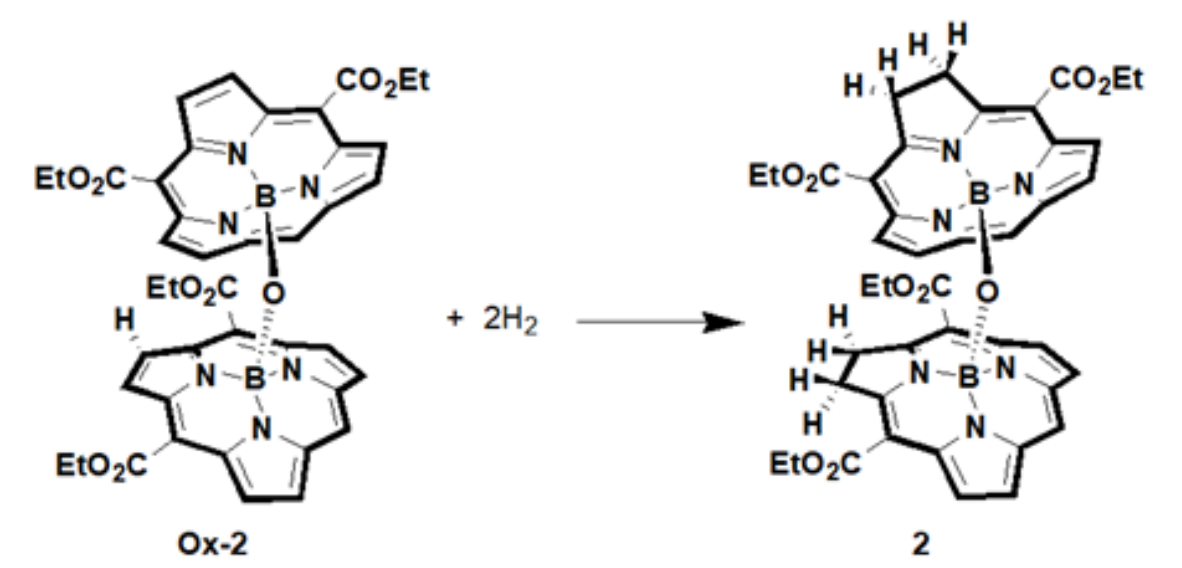

$\triangle E=-72.00 \quad(\mathrm{SR} 1)$
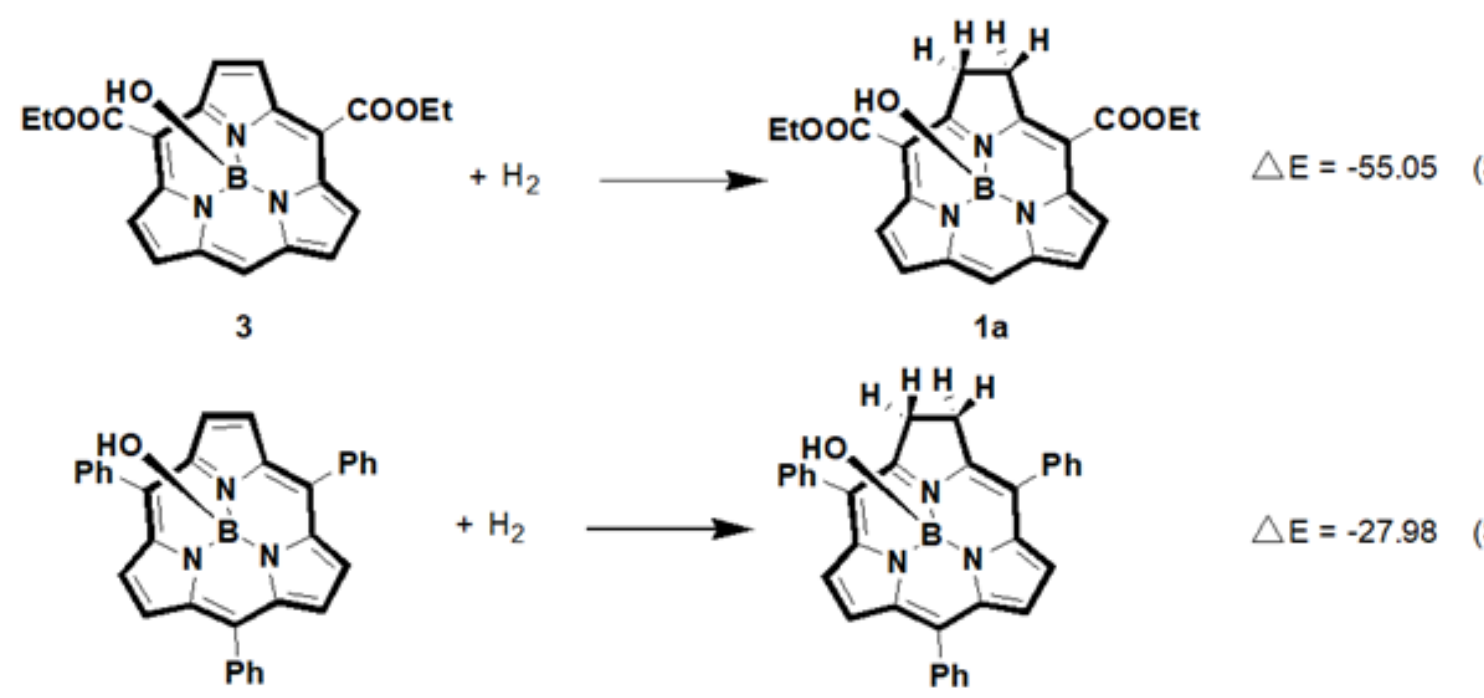

$0 x-4$
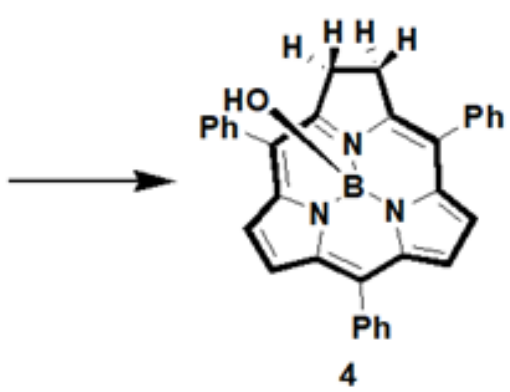

$\triangle E=-27.98 \quad(S R 3)$

To quantify the stability of the subchlorin $\mathbf{1 a}$ and its dimer $\mathbf{2}$ with regard to the corresponding subporphyrin, we have evaluated the reaction energy and compared it with that calculated for the reported meso-triphenyl subchlorin 4 . The reaction energies were calculated for macrocycles 1a, 2, and 4 at the WB97XD/def2-SVP level of theory. The reaction energies $(\Delta \mathrm{E})$ of $\mathrm{SR} 1, \mathrm{SR} 2$, and $\mathrm{SR} 3$ reactions are reported in $\mathrm{kcal} / \mathrm{mol}$. The above reaction energy $(\Delta \mathrm{E}$ in $\mathrm{kcal} / \mathrm{mol}$ ) shows that reaction is favorable in the forward direction for SR1, SR2, and SR3. Moreover, the reduction of meso-diester substituted subporphyrin dimer (Ox-2) is more feasible than its monomer 3 and meso-triphenyl substituted subporphyrin monomer (Ox-4). This trend in the reaction energy explains the exceptional stability of the subchlorin dimer $\mathbf{2}$ and its monomer analogue 1a over the reported subchlorin 4. 
The most stable subchlorin monomer 1a has dipole moment 4.0 Debye, and the most stable subchlorin dimer 2 has a negligible dipole moment 1.08 Debye. For comparing the absorption spectra of 1a with the experiment, we have considered one explicit solvent molecule (dichloromethane, $\mathrm{CH}_{2} \mathrm{Cl}_{2}$ ) and the rest of the solvent environment through the implicit solvation model. The molecular electrostatic potential (MESP) charge distribution of the monomer molecules shows that the most negative region is close to the oxygen atom. To mimic the solvent environment and to incorporate the short-range solvent effects on the chromophore, we have included one explicit dichloromethane molecule in the calculation. All the long-range solvent effects are included through the implicit solvation model SMD, as implemented in the Gaussian09 suite of packages, with dichloromethane as the solvent. The magnitude of the dipole moment is small for dimer 2 . Therefore, we have considered only the implicit solvent model in its spectral absorption calculation.

Similarly, subchlorin 4 has a dipole moment of 3.03 Debye, and here again, we have considered only the implicit solvent-model in the calculation. Table S7 reports the most intense peaks of the oxidized and the reduced species of the monomers and dimer molecules. The Q-band of the subchlorin 1a is blue-shifted compared to its dimer $\mathbf{2}$ and subchlorin $\mathbf{4}$.

Table S7 reports an analysis of the absorption spectrum, and the corresponding oscillator strength of each absorption peak is reported in brackets. The spectral patterns show the Soret-band is more intense over the Q-band of all molecules. The most intense peaks of dimer $\mathbf{2}$ are at 311.92 (1.15), 334.41 (0.169), and 490.68 (0.20) $\mathrm{nm}$. While the oxidized dimer molecule shows a single intense peak at $345.6(1.146) \mathrm{nm}$. The meso-triphenyl subchlorin 4 shows absorption maxima at 313.14 (1.27), 318.74 (0.73), 436.09 (0.39), and 497.82 (0.30) nm, respectively. On the other hand, the Ox-4 shows a single peak at $366.57(1.31) \mathrm{nm}$. The subchlorin 1a with an explicit solvent molecule shows absorption maxima at 309.51 (1.20), 331.77 (0.58), and 485.20 (0.27) nm, respectively. The Q-band of the subchlorin 1a at $485.20(0.27)$ is blue-shifted relative to its dimer $\mathbf{2}$ and the subchlorin 4 . The subporphyrin 3 shows maxima at $354.84(0.62)$ and $367.12(1.19) \mathrm{nm}$, respectively. Overall, the calculated spectrum corroborates well with the experimental spectrum.

The relative shifts and trends in the absorption spectrum of the chromophore molecules can be justified in terms of the molecular orbital (MO) picture. The HOMO-LUMO band-gap of subchlorin 1a is $2.10 \mathrm{eV}$, the corresponding Q-band in the absorption spectrum is blue-shifted 
relative to its dimer 2 (band-gap $=2.00 \mathrm{eV}$ ) and subchlorin 4 (band-gap $=2.01 \mathrm{eV}$ ), respectively. The corresponding subporphyrin 3 has a band-gap of $2.06 \mathrm{eV}$, and is further blue-shifted compared to subchlorin $\mathbf{1 a}$.

We have calculated the exciton coupling between the two-chromophores of the most stable conformer of $\mathbf{3}$. We have fragmented the disubstituted-dimer along the $\mathrm{B}-\mathrm{O}$ bond into two separate chromophores. The one with oxygen atoms is considered with a negative charge, and the other fragment a positive charge. The calculated exciton coupling constant value is $40.5 \mathrm{~cm}^{-1}$ at WB97XD/def2-SVP level of theory. The details of the theory and calculations can be found elsewhere. ${ }^{\text {S9 }}$

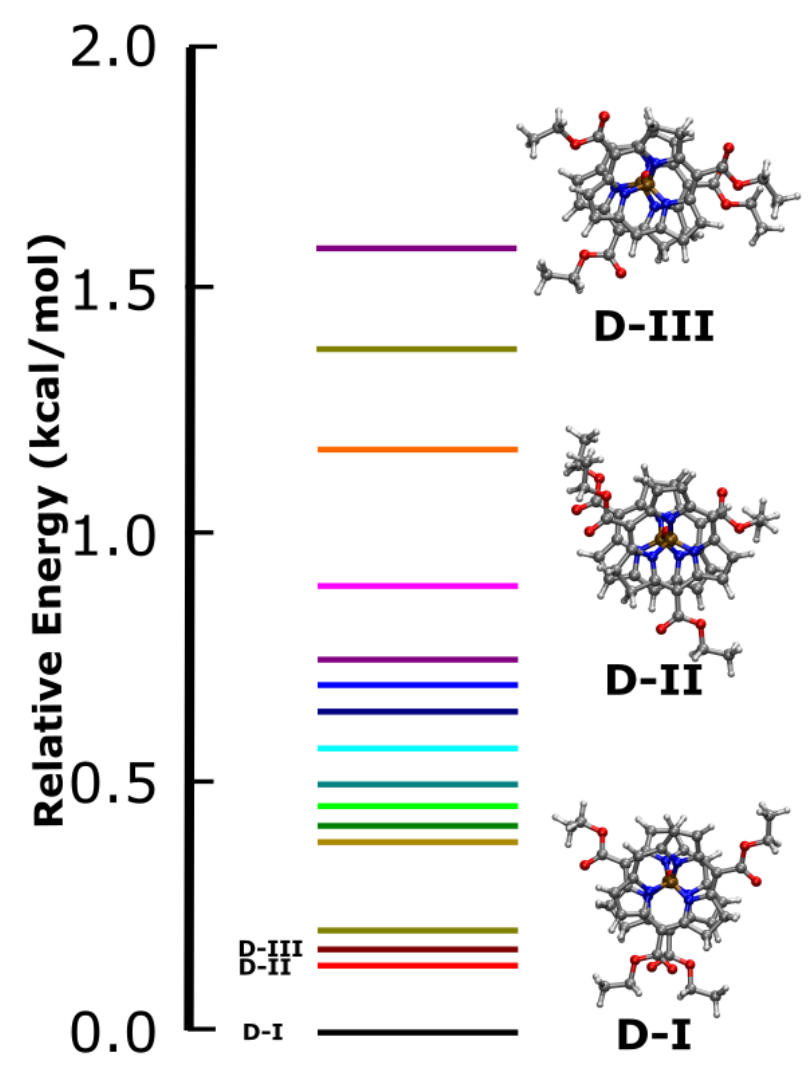

Figure S40. The relative energies of conformational isomers of dimer 2 in $\mathrm{kcal} / \mathrm{mol}$. The most stable three isomers are depicted (I-III). The relative-energy reported with respect to the most stable isomer (D-I) is depicted as zero $\mathrm{kcal} / \mathrm{mol}$. 


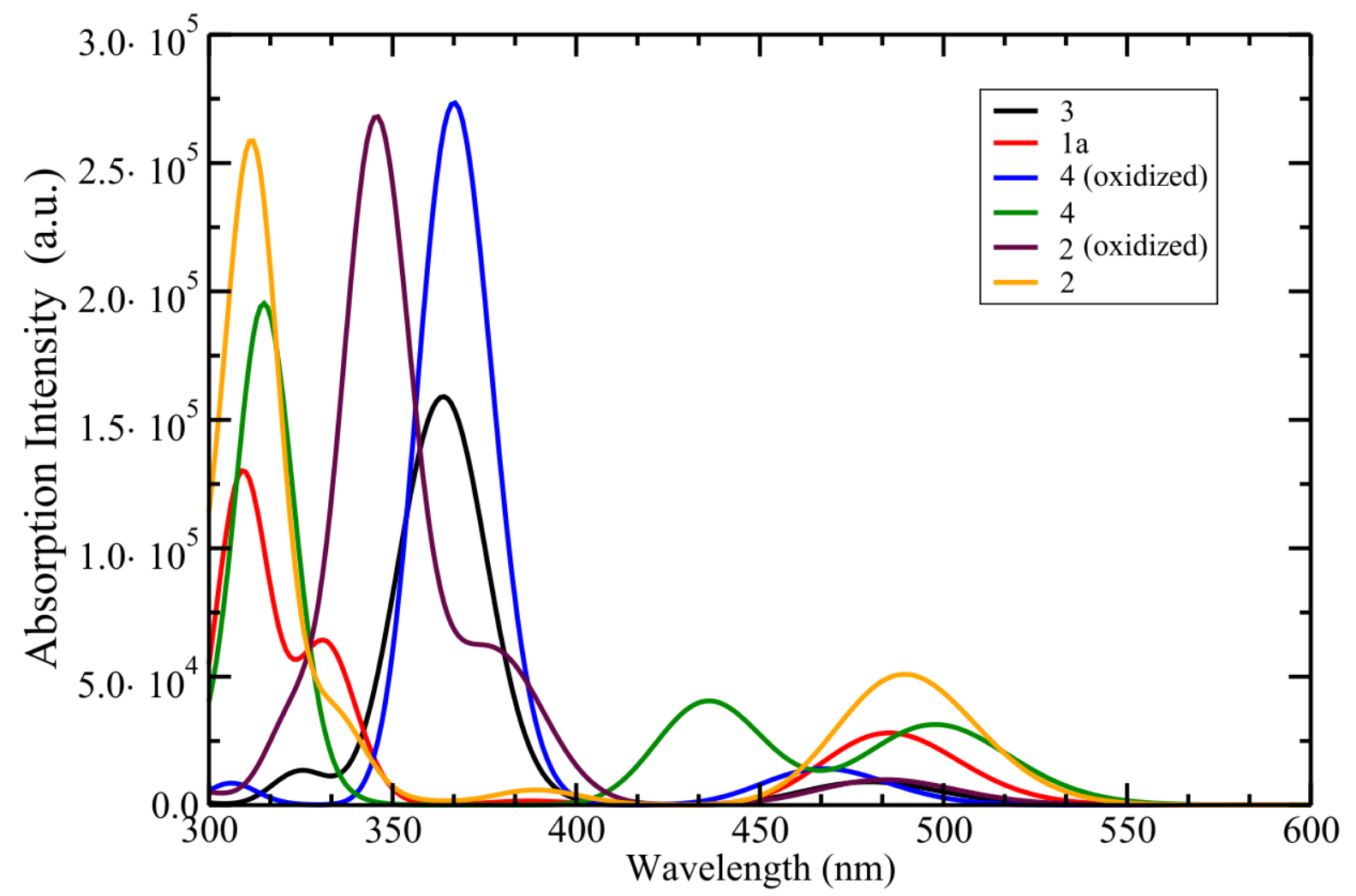

Figure S41. The absorption spectra of 3, 1a, Ox-2, 2, Ox-4 and 4 molecules evaluated at WB97XD/def2-SVP level of theory. 
Table S7. The computed absorption spectral data with $\lambda_{\max }$ in $\mathrm{nm}$ (oscillator strength) evaluated at WB97XD/def2-SVP level of theory for the reduced and oxidized molecules.

\begin{tabular}{cccr}
\hline Molecules & \multicolumn{2}{c}{ Soret-Band } & Q-band \\
\hline $\mathbf{1 a}$ & $319.81(1.03)$ & $345.27(0.64)$ & $518.78(0.30)$ \\
$\begin{array}{c}\text { 1a (explicit } \\
\mathrm{CH}_{2} \mathrm{Cl}_{2} \text { as } \\
\text { solvent) }\end{array}$ & $309.51(1.20)$ & $331.77(0.58)$ & $485.20(0.27)$ \\
$\mathbf{3}$ & & & \\
$\mathbf{3}$ (explicit & $354.84(0.62)$ & $367.12(1.19)$ & $481.61(0.08)$ \\
$\mathrm{CH}_{2} \mathrm{Cl} l_{2}$ as \\
solvent) & & & $481.35(0.08)$ \\
& & & \\
$\mathbf{4}$ & $313.14(1.27)$ & $318.74(0.73)$ & $436.09(0.39)$ \\
& & $366.57(1.31)$ & $467.72(0.08)$ \\
$\mathbf{O x}-\mathbf{4}$ & & $334.41(0.17)$ & $490.68(0.20)$ \\
$\mathbf{2}$ & $311.92(1.15)$ & & $488.57(0.29)$ \\
& $313.02(1.09)$ & $345.6(1.15)$ & \\
\hline Ox-2 & & & \\
\hline
\end{tabular}


Table S8. Atomic symbols, Cartesian coordinates (in $\AA$ ) and vibrational frequencies $\left(\mathrm{cm}^{-1}\right)$ of the structure 3 optimized at MPW1PW91/6-31g(d).

$$
\begin{aligned}
& \text { Energy }=-2337.07577 \text { Hartree } \\
& \text { Nimag }=\text { nil }
\end{aligned}
$$

\begin{tabular}{|c|c|c|c|c|c|c|}
\hline Atom & $x$ & $y$ & $z$ & \multicolumn{3}{|c|}{ Vibrational Frequencies } \\
\hline$C$ & -0.732758 & 1.651658 & -0.001735 & 0.8992 & 7.7982 & 10.8647 \\
\hline$C$ & -2.024990 & -0.243426 & 0.171590 & 22.4918 & 29.8056 & 35.6560 \\
\hline C & -2.251676 & -1.588163 & -0.129057 & 36.9527 & 53.7560 & 61.7245 \\
\hline$C$ & -1.228348 & -2.335269 & -0.826036 & 68.0347 & 78.0307 & 78.8136 \\
\hline C & 0.756252 & -2.411416 & -1.888636 & 97.6531 & 114.3980 & 118.5452 \\
\hline C & 1.875312 & -1.741098 & -2.408696 & 126.2824 & 127.7611 & 144.3131 \\
\hline $\mathrm{H}$ & 2.467705 & -2.216052 & -3.181918 & 150.5783 & 168.8620 & 194.6586 \\
\hline C & 2.121296 & -0.400067 & -2.078987 & 214.9068 & 219.7365 & 233.2338 \\
\hline C & 2.852505 & 0.658422 & -2.696175 & 258.2323 & 263.5487 & 271.7610 \\
\hline $\mathrm{H}$ & 3.569695 & 0.540561 & -3.496408 & 274.7315 & 283.7769 & 291.7194 \\
\hline C & 2.414729 & 1.852535 & -2.153309 & 346.0902 & 353.6857 & 370.0133 \\
\hline $\mathrm{H}$ & 2.714596 & 2.850918 & -2.426765 & 371.1294 & 379.0835 & 385.8061 \\
\hline C & 1.407101 & 1.540050 & -1.187276 & 418.4659 & 422.5449 & 424.0885 \\
\hline C & 0.397822 & 2.305792 & -0.492253 & 435.3323 & 440.6717 & 474.5049 \\
\hline C & -3.553316 & -2.244608 & 0.080902 & 487.4151 & 493.2318 & 533.5305 \\
\hline$C$ & -5.724735 & -2.062621 & 0.961096 & 561.9482 & 576.4774 & 631.6626 \\
\hline $\mathrm{H}$ & -5.583158 & -2.980585 & 1.537648 & 668.8526 & 695.5027 & 713.3779 \\
\hline $\mathrm{H}$ & -6.165203 & -2.344587 & 0.000890 & 722.5201 & 736.6473 & 745.6908 \\
\hline C & -6.573431 & -1.054881 & 1.700181 & 758.2098 & 774.1379 & 774.2409 \\
\hline $\mathrm{H}$ & -6.116738 & -0.783121 & 2.655511 & 779.7417 & 783.7471 & 789.3027 \\
\hline $\mathrm{H}$ & -7.560413 & -1.480052 & 1.902081 & 807.9338 & 809.3345 & 816.9375 \\
\hline $\mathrm{H}$ & -6.708413 & -0.144968 & 1.109372 & 820.4785 & 821.0702 & 837.1945 \\
\hline C & 0.540144 & 3.771751 & -0.479297 & 840.5218 & 871.9540 & 886.4826 \\
\hline$N$ & -0.795807 & 0.296479 & -0.019221 & 894.5221 & 904.8776 & 922.5074 \\
\hline $\mathrm{N}$ & -0.011980 & -1.769906 & -0.946874 & 928.5661 & 934.2226 & 949.4529 \\
\hline
\end{tabular}




\begin{tabular}{|c|c|c|c|c|c|c|}
\hline $\mathrm{N}$ & 1.325817 & 0.199388 & -1.133195 & 968.0706 & 997.1334 & 1028.9954 \\
\hline O & -3.817940 & -3.375794 & -0.269076 & 1032.1427 & 1045.2957 & 1054.8228 \\
\hline O & -4.442345 & -1.462131 & 0.725851 & 1074.7257 & 1076.1399 & 1081.4154 \\
\hline O & 1.453331 & 4.379321 & -0.997999 & 1088.3597 & 1096.6932 & 1109.6499 \\
\hline O & -0.452281 & 4.393439 & 0.189212 & 1145.0283 & 1157.5851 & 1157.9196 \\
\hline B & 0.457031 & -0.564154 & -0.188006 & 1173.1602 & 1185.8069 & 1196.7906 \\
\hline C & -0.349787 & 5.824117 & 0.240190 & 1197.0459 & 1201.7740 & 1237.2731 \\
\hline $\mathrm{H}$ & -0.342604 & 6.215513 & -0.780764 & 1243.2068 & 1254.6768 & 1257.5933 \\
\hline $\mathrm{H}$ & 0.605221 & 6.092285 & 0.699655 & 1266.5251 & 1291.5125 & 1297.2060 \\
\hline C & -1.525590 & 6.339043 & 1.036730 & 1314.6500 & 1314.7275 & 1316.3086 \\
\hline $\mathrm{H}$ & -1.478813 & 7.429559 & 1.102109 & 1331.7064 & 1360.5622 & 1371.3758 \\
\hline $\mathrm{H}$ & -2.472220 & 6.066081 & 0.562742 & 1384.1923 & 1413.3361 & 1414.3415 \\
\hline $\mathrm{H}$ & -1.517593 & 5.935227 & 2.052556 & 1422.7906 & 1424.1752 & 1442.3271 \\
\hline O & 1.139941 & -0.821549 & 1.047046 & 1443.6157 & 1454.1830 & 1458.8830 \\
\hline $\mathrm{H}$ & 3.219146 & -0.937868 & 1.444784 & 1481.8872 & 1485.0607 & 1487.1009 \\
\hline $\mathrm{H}$ & 0.705577 & -1.520913 & 1.548680 & 1489.9771 & 1509.8520 & 1515.1119 \\
\hline C & -1.223923 & -3.502249 & -1.650706 & 1520.7271 & 1520.8408 & 1523.8290 \\
\hline C & -0.002894 & -3.545659 & -2.300507 & 1530.2330 & 1530.7169 & 1549.7586 \\
\hline $\mathrm{H}$ & -2.057848 & -4.174730 & -1.768340 & 1549.8123 & 1557.7980 & 1564.5184 \\
\hline $\mathrm{H}$ & 0.303891 & -4.268739 & -3.043314 & 1573.8813 & 1613.6484 & 1614.6480 \\
\hline C & -2.944724 & 0.877576 & 0.597704 & 1818.9414 & 1821.8324 & 3087.2744 \\
\hline H & -3.830195 & 0.918858 & -0.039509 & 3087.3900 & 3103.3533 & 3103.4700 \\
\hline $\mathrm{H}$ & -3.307883 & 0.710195 & 1.614346 & 3118.6164 & 3126.0980 & 3143.3924 \\
\hline C & -2.071934 & 2.154224 & 0.485603 & 3146.3986 & 3146.4499 & 3156.8680 \\
\hline $\mathrm{H}$ & -1.960244 & 2.665942 & 1.444457 & 3170.2814 & 3170.4431 & 3172.3026 \\
\hline $\mathrm{H}$ & -2.490795 & 2.887961 & -0.205441 & 3175.5589 & 3175.6222 & 3239.3036 \\
\hline C & 4.245527 & -1.032428 & 1.799163 & 3257.0373 & 3288.8449 & 3289.6428 \\
\hline $\mathrm{H}$ & 4.786615 & -0.092028 & 1.735131 & 3336.0001 & 3336.3088 & 3847.1623 \\
\hline $\mathrm{Cl}$ & 5.091170 & -2.216865 & 0.778094 & & & \\
\hline $\mathrm{Cl}$ & 4.193957 & -1.513511 & 3.511574 & & & \\
\hline
\end{tabular}


Table S9. Atomic symbols, Cartesian coordinates (in $\AA$ ) and vibrational frequencies $\left(\mathrm{cm}^{-1}\right)$ of the structure 1a optimized at MPW1PW91/6-31g(d).

Energy $=-2335.85853$ Hartree

Nimag $=$ nil

\begin{tabular}{|c|c|c|c|c|c|c|}
\hline \multirow{2}{*}{$\begin{array}{c}\text { Atom } \\
\mathrm{C}\end{array}$} & \multirow{2}{*}{$\begin{array}{c}\mathrm{m} \quad \mathrm{x} \\
-1.429811\end{array}$} & \multirow{2}{*}{$\begin{array}{c}y \\
1.332787\end{array}$} & \multirow{2}{*}{$\begin{array}{c}z \\
0.140387\end{array}$} & \multicolumn{3}{|c|}{ Vibrational Frequencies } \\
\hline & & & & 4.6339 & 8.3750 & 11.4954 \\
\hline$C$ & -1.788622 & -0.901097 & 0.132178 & 20.8739 & 25.9617 & 33.6771 \\
\hline C & -1.415832 & -2.208549 & -0.273619 & 40.2385 & 54.3491 & 63.3571 \\
\hline C & -0.165722 & -2.390870 & -0.933425 & 65.1979 & 74.0617 & 77.3073 \\
\hline C & 1.680040 & -1.537535 & -1.899612 & 98.1040 & 116.4175 & 120.4462 \\
\hline C & 2.425292 & -0.424921 & -2.325186 & 127.7766 & 132.5351 & 150.8003 \\
\hline H & 3.176116 & -0.545491 & -3.096271 & 164.4429 & 190.8600 & 212.8289 \\
\hline C & 2.056381 & 0.865736 & -1.908463 & 222.9447 & 229.1993 & 259.3427 \\
\hline C & 2.272296 & 2.183268 & -2.438022 & 265.0371 & 271.4582 & 276.6538 \\
\hline $\mathrm{H}$ & 2.976563 & 2.435843 & -3.218095 & 288.0922 & 291.7631 & 315.8373 \\
\hline $\mathrm{C}$ & 1.366190 & 3.031024 & -1.844601 & 354.9134 & 363.5371 & 376.0593 \\
\hline $\mathrm{H}$ & 1.206749 & 4.076751 & -2.051030 & 387.5712 & 391.5760 & 418.0482 \\
\hline C & 0.569291 & 2.248242 & -0.934733 & 420.2451 & 431.6544 & 437.6520 \\
\hline$C$ & -0.672378 & 2.459593 & -0.268538 & 443.4254 & 483.0410 & 486.9288 \\
\hline$C$ & -2.332647 & -3.367003 & -0.197813 & 497.6936 & 501.4415 & 540.2788 \\
\hline$C$ & -4.370599 & -4.202590 & 0.615651 & 569.0109 & 675.5870 & 681.0550 \\
\hline $\mathrm{H}$ & -3.853885 & -5.041186 & 1.089780 & 684.4496 & 716.9844 & 718.5724 \\
\hline $\mathrm{H}$ & -4.667909 & -4.524515 & -0.386081 & 722.2899 & 740.0311 & 748.0767 \\
\hline$C$ & -5.547995 & -3.727101 & 1.433602 & 758.2889 & 771.4499 & 777.9027 \\
\hline $\mathrm{H}$ & -5.227237 & -3.394489 & 2.424055 & 786.0578 & 792.3356 & 809.4568 \\
\hline $\mathrm{H}$ & -6.263064 & -4.544493 & 1.560935 & 813.8996 & 815.8889 & 818.1632 \\
\hline $\mathrm{H}$ & -6.062846 & -2.898876 & 0.939286 & 820.6703 & 821.2725 & 825.5904 \\
\hline$C$ & -1.186026 & 3.844934 & -0.189131 & 838.9631 & 880.6025 & 886.0628 \\
\hline$N$ & -0.864071 & 0.096241 & 0.062422 & 900.3677 & 914.3934 & 932.8382 \\
\hline N & 0.711308 & -1.359036 & -0.956940 & 937.7530 & 939.7569 & 947.4302 \\
\hline
\end{tabular}




\begin{tabular}{|c|c|c|c|c|c|c|}
\hline$N$ & 1.085608 & 0.998007 & -0.962373 & 952.1630 & 980.0849 & 1010.1787 \\
\hline $\mathrm{O}$ & -2.105996 & -4.454611 & -0.685265 & 1067.3410 & 1070.5353 & 1073.9674 \\
\hline 0 & -3.452172 & -3.104560 & 0.497309 & 1086.1054 & 1089.8190 & 1091.6194 \\
\hline $\mathrm{O}$ & -0.642854 & 4.807945 & -0.688513 & 1097.1877 & 1099.7999 & 1144.1823 \\
\hline 0 & -2.320717 & 3.942715 & 0.523445 & 1151.3669 & 1158.4763 & 1160.3280 \\
\hline B & 0.618010 & -0.127246 & -0.092442 & 1196.5210 & 1196.5755 & 1205.7046 \\
\hline C & -2.851613 & 5.271238 & 0.649580 & 1218.2714 & 1222.0481 & 1241.9160 \\
\hline $\mathrm{H}$ & -3.053746 & 5.667887 & -0.349128 & 1260.7534 & 1268.1199 & 1293.9403 \\
\hline $\mathrm{H}$ & -2.091658 & 5.909204 & 1.108311 & 1315.5141 & 1315.5518 & 1332.6113 \\
\hline$C$ & -4.102413 & 5.185084 & 1.491905 & 1340.4331 & 1358.9444 & 1378.3472 \\
\hline $\mathrm{H}$ & -4.526486 & 6.183989 & 1.626220 & 1392.6532 & 1402.4123 & 1407.8438 \\
\hline $\mathrm{H}$ & -4.858052 & 4.556523 & 1.013011 & 1417.7892 & 1424.2990 & 1440.4726 \\
\hline $\mathrm{H}$ & -3.881122 & 4.770567 & 2.478649 & 1450.3609 & 1455.8076 & 1461.0764 \\
\hline 0 & 1.376920 & -0.157568 & 1.125990 & 1464.5060 & 1495.8869 & 1498.9412 \\
\hline $\mathrm{H}$ & 3.447720 & 0.062946 & 1.494326 & 1509.2840 & 1512.3299 & 1520.5635 \\
\hline $\mathrm{H}$ & 1.111310 & -0.896547 & 1.685652 & 1520.5868 & 1523.1964 & 1529.7029 \\
\hline C & 0.358096 & -3.386165 & -1.833858 & 1529.9902 & 1544.9097 & 1548.8179 \\
\hline C & 1.483640 & -2.860390 & -2.423476 & 1549.5663 & 1567.9929 & 1570.4624 \\
\hline $\mathrm{H}$ & -0.113017 & -4.333958 & -2.037182 & 1587.7426 & 1599.5363 & 1816.6868 \\
\hline $\mathrm{H}$ & 2.080952 & -3.321278 & -3.197547 & 1819.4493 & 3088.1047 & 3088.1900 \\
\hline C & 4.456722 & 0.032731 & 1.905924 & 3103.5582 & 3103.7818 & 3141.1780 \\
\hline $\mathrm{H}$ & 4.747420 & 0.982167 & 2.347800 & 3147.3600 & 3147.4967 & 3170.4322 \\
\hline $\mathrm{Cl}$ & 5.600006 & -0.327643 & 0.593062 & 3170.6426 & 3177.6326 & 3177.7380 \\
\hline $\mathrm{Cl}$ & 4.479125 & -1.194199 & 3.194753 & 3238.7095 & 3263.8627 & 3291.5621 \\
\hline$C$ & -2.826629 & 1.104246 & 0.418058 & 3292.0589 & 3327.6421 & 3337.2968 \\
\hline C & -3.044098 & -0.249293 & 0.414219 & 3337.7505 & 3342.9847 & 3844.0827 \\
\hline $\mathrm{H}$ & -3.566665 & 1.878418 & 0.536285 & & & \\
\hline $\mathrm{H}$ & -3.989133 & -0.753967 & 0.528719 & & & \\
\hline
\end{tabular}


Table S10. Atomic symbols, Cartesian coordinates (in $\AA$ ) and vibrational frequencies $\left(\mathrm{cm}^{-1}\right)$ of the structure Ox-4 optimized at MPW1PW91/6-31g(d).

$$
\begin{aligned}
& \text { Energy }=-1534.89851 \text { Hartree } \\
& \text { Nimag }=\text { nil }
\end{aligned}
$$

\begin{tabular}{|c|c|c|c|c|c|c|}
\hline \multicolumn{2}{|c|}{ Atom } & \multirow{2}{*}{$\frac{y}{2.213634}$} & \multirow{2}{*}{$\begin{array}{c}\mathbf{z} \\
0.305956\end{array}$} & \multicolumn{3}{|c|}{ Vibrational Frequencies } \\
\hline C & 0.773620 & & & 28.9501 & 41.2511 & 41.3198 \\
\hline C & 2.033210 & 2.761444 & -0.119611 & 47.7243 & 48.4327 & 58.8105 \\
\hline $\mathrm{H}$ & 2.192726 & 3.778005 & -0.448948 & 71.0840 & 71.9691 & 90.4023 \\
\hline C & 2.958830 & 1.742521 & -0.115270 & 139.0752 & 141.9165 & 142.2566 \\
\hline $\mathrm{H}$ & 3.991933 & 1.814091 & -0.421311 & 158.1682 & 158.9049 & 212.7846 \\
\hline C & 2.286872 & 0.537095 & 0.292591 & 217.4612 & 220.6735 & 228.0825 \\
\hline C & 2.598512 & -0.845706 & 0.216755 & 241.4992 & 258.4322 & 262.9484 \\
\hline C & 1.530494 & -1.776955 & 0.299425 & 284.4440 & 324.0815 & 328.9838 \\
\hline C & 1.375252 & -3.136970 & -0.140564 & 329.5072 & 362.3286 & 374.8983 \\
\hline $\mathrm{H}$ & 2.176221 & -3.779810 & -0.475827 & 418.7286 & 418.9366 & 421.7470 \\
\hline C & 0.030221 & -3.430064 & -0.138131 & 433.2547 & 434.2322 & 458.2482 \\
\hline $\mathrm{H}$ & -0.424314 & -4.357746 & -0.452312 & 477.3182 & 478.5000 & 488.1326 \\
\hline C & -0.677624 & -2.249270 & 0.281473 & 500.3008 & 501.2054 & 520.1996 \\
\hline C & -2.031491 & -1.826857 & 0.217852 & 562.9432 & 584.0357 & 585.3119 \\
\hline C & -2.303106 & -0.436959 & 0.312675 & 618.4165 & 634.2178 & 634.3237 \\
\hline C & -3.408077 & 0.378330 & -0.113054 & 638.2708 & 654.6162 & 679.2916 \\
\hline $\mathrm{H}$ & -4.368971 & 0.006732 & -0.438511 & 683.4411 & 683.8902 & 693.2900 \\
\hline C & -2.990343 & 1.690037 & -0.112440 & 693.5775 & 719.3917 & 719.7683 \\
\hline $\mathrm{H}$ & -3.571035 & 2.547575 & -0.418134 & 720.8505 & 736.5156 & 737.5474 \\
\hline C & -1.608354 & 1.712111 & 0.289505 & 753.2193 & 767.4555 & 771.2911 \\
\hline C & -0.566531 & 2.674760 & 0.217912 & 777.4979 & 797.2437 & 801.6899 \\
\hline N & 1.019503 & 0.913216 & 0.614639 & 811.7480 & 817.9607 & 824.2657 \\
\hline N & 0.281860 & -1.344310 & 0.608234 & 837.3301 & 872.0086 & 872.4772 \\
\hline N & -1.299216 & 0.427658 & 0.615077 & 872.6389 & 886.9254 & 891.3038 \\
\hline B & 0.003469 & -0.014002 & 1.233679 & 920.3647 & 921.2027 & 921.8487 \\
\hline
\end{tabular}




\begin{tabular}{|c|c|c|c|c|c|c|}
\hline $\mathrm{O}$ & 0.024909 & -0.118592 & 2.657107 & 923.5844 & 933.4711 & 941.9851 \\
\hline C & 3.956549 & -1.328136 & -0.105004 & 946.6323 & 955.1140 & 969.2509 \\
\hline C & 4.686149 & -0.817820 & -1.187381 & 987.6124 & 987.7449 & 988.0749 \\
\hline $\mathrm{H}$ & 4.234195 & -0.067181 & -1.826972 & 1009.2805 & 1009.4218 & 1009.5880 \\
\hline C & 5.960814 & -1.294178 & -1.471817 & 1022.1679 & 1022.3229 & 1022.6594 \\
\hline C & 6.528705 & -2.293969 & -0.686419 & 1057.6447 & 1064.4046 & 1065.9432 \\
\hline $\mathrm{H}$ & 7.523521 & -2.665310 & -0.910563 & 1067.3885 & 1072.3481 & 1084.7746 \\
\hline C & 5.809659 & -2.817990 & 0.384052 & 1092.4341 & 1094.3232 & 1103.7733 \\
\hline C & 4.535903 & -2.340471 & 0.672257 & 1104.0509 & 1109.1754 & 1122.3916 \\
\hline $\mathrm{H}$ & 3.983735 & -2.735200 & 1.519129 & 1122.5643 & 1122.7482 & 1190.0986 \\
\hline C & -3.130093 & -2.759380 & -0.104620 & 1198.2832 & 1198.3273 & 1198.4570 \\
\hline C & -3.058817 & -3.637007 & -1.195025 & 1206.3341 & 1220.7433 & 1220.9062 \\
\hline $\mathrm{H}$ & -2.186411 & -3.614972 & -1.839379 & 1221.3393 & 1233.0832 & 1253.5742 \\
\hline C & -4.109645 & -4.501139 & -1.480609 & 1259.3130 & 1315.0599 & 1331.1902 \\
\hline C & -5.254808 & -4.500795 & -0.688441 & 1346.1994 & 1346.7489 & 1361.6728 \\
\hline $\mathrm{H}$ & -6.074509 & -5.175522 & -0.913400 & 1365.3871 & 1368.3061 & 1380.9805 \\
\hline C & -5.343285 & -3.625570 & 0.390269 & 1381.0605 & 1381.0742 & 1402.2776 \\
\hline C & -4.291712 & -2.763036 & 0.679635 & 1407.4290 & 1409.4402 & 1447.7952 \\
\hline $\mathrm{H}$ & -4.352709 & -2.095742 & 1.533375 & 1447.8508 & 1455.4285 & 1490.5712 \\
\hline C & -0.826755 & 4.092085 & -0.104860 & 1491.3338 & 1497.3166 & 1498.0151 \\
\hline C & -1.642048 & 4.466855 & -1.181721 & 1499.1958 & 1509.6836 & 1519.0268 \\
\hline $\mathrm{H}$ & -2.072034 & 3.698661 & -1.815423 & 1520.0202 & 1553.6973 & 1553.8591 \\
\hline C & -1.867859 & 5.808125 & -1.468788 & 1554.0159 & 1557.1840 & 1571.7533 \\
\hline C & -1.278015 & 6.802271 & -0.692468 & 1571.9823 & 1601.2890 & 1657.5653 \\
\hline $\mathrm{H}$ & -1.454481 & 7.848842 & -0.918855 & 1657.6235 & 1657.6625 & 1685.6860 \\
\hline C & -0.455296 & 6.444231 & 0.371619 & 1685.7480 & 1685.9864 & 3213.7153 \\
\hline C & -0.231903 & 5.103011 & 0.662631 & 3213.7413 & 3213.8212 & 3221.0557 \\
\hline $\mathrm{H}$ & 0.395597 & 4.826209 & 1.503938 & 3221.4067 & 3221.5080 & 3230.9384 \\
\hline $\mathrm{H}$ & 6.507302 & -0.891586 & -2.318952 & 3231.5379 & 3231.6161 & 3237.9093 \\
\hline $\mathrm{H}$ & 6.243710 & -3.596981 & 1.002902 & 3237.9314 & 3238.0147 & 3244.2251 \\
\hline $\mathrm{H}$ & 0.009659 & 7.211123 & 0.983107 & 3244.2935 & 3244.5985 & 3291.6142 \\
\hline $\mathrm{H}$ & -2.496800 & 6.077580 & -2.311489 & 3291.7317 & 3292.4542 & 3308.8675 \\
\hline
\end{tabular}



H $-4.038580-5.168518-2.333779$
H $\quad-6.231017-3.618457 \quad 1.014849$
H $\quad-0.153569 \quad 0.735576 \quad 3.065331$

$3309.0062 \quad 3309.7650 \quad 3851.9107$

Table S11. Atomic symbols, Cartesian coordinates (in $\AA$ ) and vibrational frequencies $\left(\mathrm{cm}^{-1}\right)$ of the structure 4 optimized at MPW1PW91/6-31g(d).

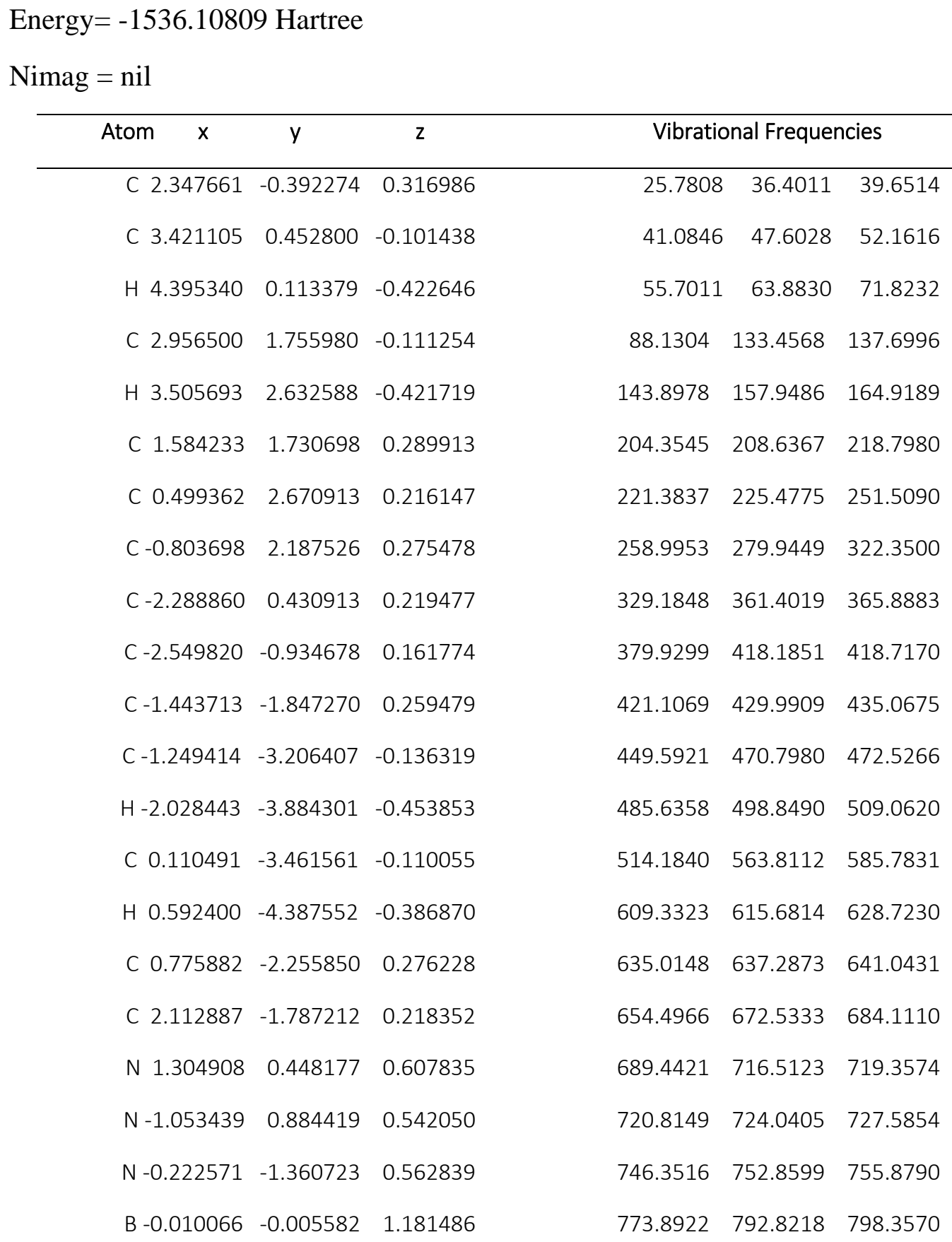




\begin{tabular}{|c|c|c|c|c|c|}
\hline O -0.155379 & 0.080914 & 2.599444 & 802.4942 & 807.7755 & 823.0685 \\
\hline С 0.744568 & 4.097742 & -0.100573 & 834.1703 & 864.3012 & 869.6243 \\
\hline C 1.455575 & 4.477245 & -1.246610 & 871.8903 & 872.4147 & 873.7169 \\
\hline H 1.823391 & 3.710030 & -1.920417 & 906.6666 & 907.6016 & 918.3171 \\
\hline C 1.671369 & 5.820041 & -1.538068 & 924.0175 & 940.6152 & 945.9495 \\
\hline C 1.173817 & 6.809839 & -0.694684 & 948.6802 & 973.3265 & 982.8301 \\
\hline H 1.340989 & 7.857444 & -0.923876 & 986.2256 & 988.5590 & 998.8759 \\
\hline C 0.460377 & 6.446729 & 0.444025 & 1006.6851 & 1007.8788 & 1009.9132 \\
\hline C 0.250953 & 5.103609 & 0.739037 & 1022.3549 & 1022.4591 & 1022.8143 \\
\hline H - -0.281957 & 4.821752 & 1.642024 & 1027.7490 & 1029.4128 & 1042.9406 \\
\hline C-3.900926 & -1.473408 & -0.125111 & 1061.3096 & 1066.4772 & 1067.7788 \\
\hline C -4.617168 & -1.112471 & -1.272488 & 1078.5500 & 1085.3517 & 1089.7100 \\
\hline H -4.166137 & -0.437514 & -1.992703 & 1097.1366 & 1106.3785 & 1119.2604 \\
\hline C -5.881068 & -1.637964 & -1.519695 & 1120.0028 & 1122.9832 & 1182.0775 \\
\hline C - 6.449205 & -2.545092 & -0.629683 & 1190.9560 & 1192.2511 & 1197.4772 \\
\hline H -7.434443 & -2.956834 & -0.823861 & 1197.6463 & 1198.3700 & 1218.5473 \\
\hline C - 5.741407 & -2.925949 & 0.506929 & 1218.8493 & 1219.1581 & 1222.1317 \\
\hline C -4.480384 & -2.394970 & 0.756656 & 1247.5352 & 1256.2857 & 1262.8617 \\
\hline H -3.934446 & -2.682969 & 1.649547 & 1288.7511 & 1315.6516 & 1317.4254 \\
\hline C 3.241495 & -2.688262 & -0.088799 & 1339.5538 & 1345.8777 & 1346.9645 \\
\hline C 3.191766 & -3.594454 & -1.156804 & 1354.7355 & 1364.1002 & 1378.8759 \\
\hline H 2.314032 & -3.616932 & -1.793419 & 1379.3840 & 1381.1219 & 1385.8822 \\
\hline C 4.269182 & -4.429269 & -1.429609 & 1405.7480 & 1425.8055 & 1439.7570 \\
\hline C 5.418441 & -4.372775 & -0.645399 & 1449.5629 & 1468.4160 & 1473.0020 \\
\hline H 6.258516 & -5.025489 & -0.859961 & 1485.6216 & 1490.7984 & 1498.8391 \\
\hline C 5.485082 & -3.469152 & 0.411271 & 1499.4871 & 1500.8339 & 1517.0030 \\
\hline C 4.408206 & -2.633740 & 0.686125 & 1522.3254 & 1546.5596 & 1553.7362 \\
\hline H 4.455360 & -1.939950 & 1.519275 & 1557.0192 & 1560.4535 & 1566.3979 \\
\hline H 2.221776 & 6.093954 & -2.432850 & 1568.1510 & 1587.2485 & 1622.7772 \\
\hline H 0.073512 & 7.210844 & 1.111082 & 1657.9673 & 1659.0971 & 1659.6919 \\
\hline
\end{tabular}




\begin{tabular}{|c|c|c|c|c|c|}
\hline H 6.376430 & -3.417108 & 1.028519 & 1685.7320 & 1687.3283 & 1687.8320 \\
\hline H 4.214942 & -5.118742 & -2.266260 & 3101.2145 & 3108.7465 & 3143.2696 \\
\hline H -6.417676 & -1.347677 & -2.417679 & 3164.7881 & 3211.0220 & 3211.2678 \\
\hline H -6.174053 & -3.635096 & 1.205777 & 3213.9797 & 3217.4480 & 3218.2918 \\
\hline C - 2.122995 & 2.830305 & -0.098150 & 3222.0740 & 3227.1531 & 3228.1577 \\
\hline H - 2.407252 & 3.604961 & 0.618502 & 3232.2817 & 3233.6344 & 3234.1354 \\
\hline H -2.056702 & 3.317377 & -1.075276 & 3239.5635 & 3241.8814 & 3242.1720 \\
\hline C - 3.136584 & 1.647695 & -0.095761 & 3245.2887 & 3289.3407 & 3290.1770 \\
\hline H -3.655483 & 1.557399 & -1.051154 & 3305.9822 & 3306.7651 & 3853.8282 \\
\hline H -3.908683 & 1.779007 & 0.668872 & & & \\
\hline H 0.501722 & -0.466760 & 3.042630 & & & \\
\hline
\end{tabular}

Table S12. Atomic symbols, Cartesian coordinates (in $\AA$ ) and vibrational frequencies $\left(\mathrm{cm}^{-1}\right)$ of the structure Ox-2 optimized at MPW1PW91/6-31g(d).

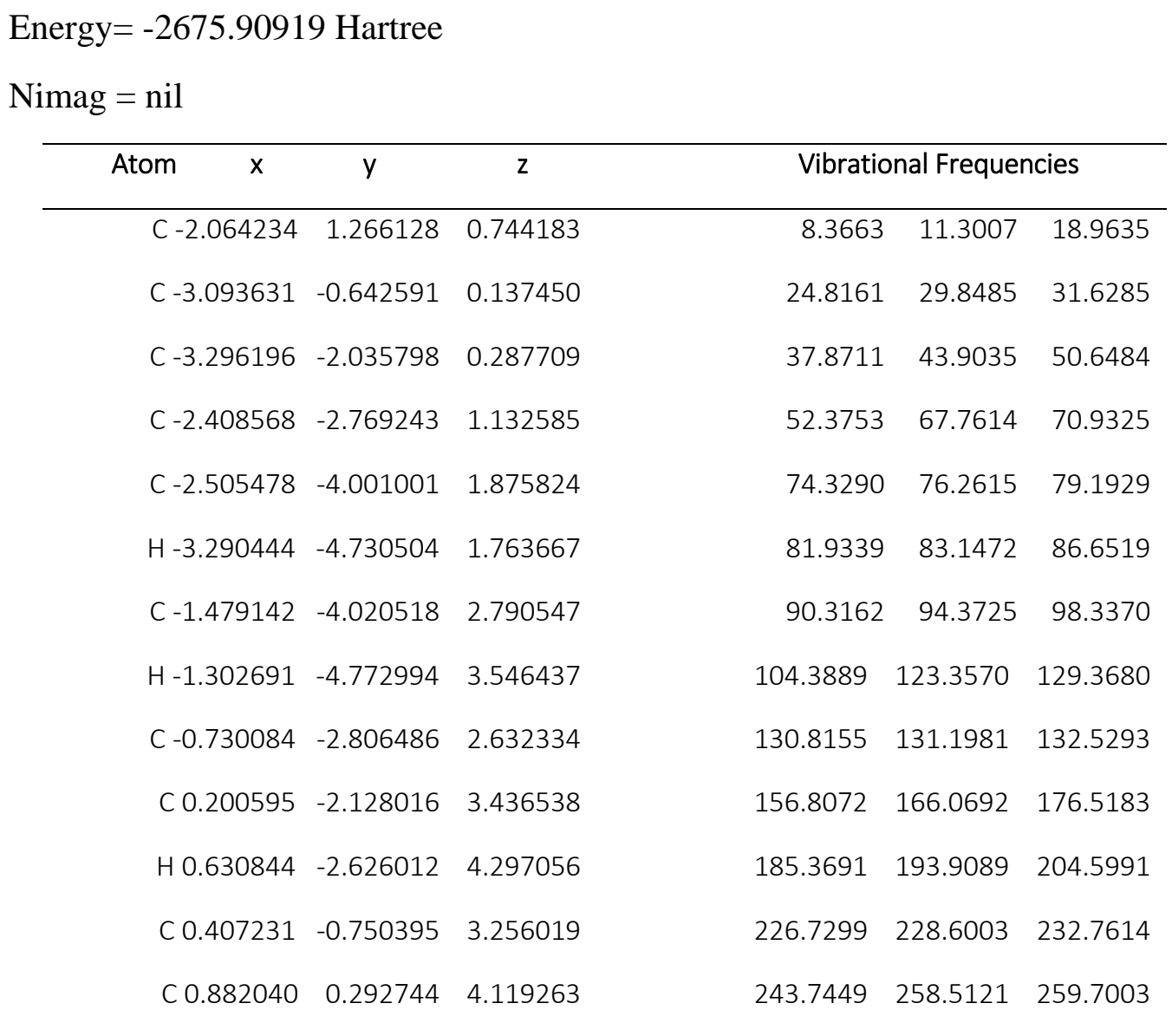




\begin{tabular}{|c|c|c|c|c|c|}
\hline H 1.388916 & 0.143851 & 5.062404 & 261.9162 & 265.7652 & 271.0057 \\
\hline C 0.491290 & 1.494266 & 3.578227 & 274.5705 & 277.8326 & 290.3246 \\
\hline H 0.631714 & 2.475171 & 4.001019 & 309.5746 & 313.3080 & 317.1934 \\
\hline C - -0.235013 & 1.214021 & 2.365737 & 321.9316 & 334.6806 & 364.3599 \\
\hline C - 1.129686 & 1.964455 & 1.545055 & 366.4379 & 372.6807 & 377.6262 \\
\hline C - 1.214519 & 3.440401 & 1.590716 & 393.5057 & 399.7569 & 405.1404 \\
\hline C - 0.218722 & 5.428598 & 2.346698 & 415.8853 & 418.7958 & 422.0203 \\
\hline H -1.188995 & 5.817914 & 2.666747 & 423.9525 & 430.5138 & 430.8819 \\
\hline H -0.041214 & 5.753043 & 1.319011 & 432.8630 & 444.8604 & 449.8022 \\
\hline C 0.897456 & 5.851407 & 3.272375 & 453.8817 & 459.2890 & 487.5782 \\
\hline H 0.709198 & 5.519299 & 4.297141 & 492.8188 & 496.4234 & 500.3185 \\
\hline H 0.980118 & 6.941883 & 3.277196 & 501.5638 & 504.2651 & 505.6040 \\
\hline H 1.852942 & 5.436632 & 2.941735 & 515.8824 & 518.8460 & 523.2320 \\
\hline O -2.032959 & 4.100191 & 0.979399 & 621.4507 & 674.4599 & 674.5057 \\
\hline O -0.266314 & 3.992547 & 2.358240 & 680.0206 & 681.4516 & 684.9859 \\
\hline C 1.651815 & 1.835852 & -0.727688 & 685.4872 & 716.5147 & 717.7104 \\
\hline C 3.189078 & 0.287594 & -0.170226 & 718.4803 & 718.6965 & 736.6428 \\
\hline C 3.784486 & -0.982669 & -0.360254 & 743.1857 & 747.7510 & 753.6895 \\
\hline C 3.144151 & -1.915087 & -1.231850 & 767.9161 & 770.4336 & 772.5513 \\
\hline C 3.590951 & -3.043076 & -2.010691 & 777.3036 & 779.3205 & 780.9851 \\
\hline H 4.553512 & -3.517780 & -1.915613 & 784.8779 & 796.0792 & 800.8156 \\
\hline C 2.611702 & -3.330541 & -2.932140 & 802.6095 & 803.2806 & 809.3840 \\
\hline H 2.658289 & -4.078616 & -3.711204 & 811.4460 & 812.7548 & 820.5003 \\
\hline C 1.544324 & -2.389940 & -2.742800 & 821.9357 & 825.6039 & 827.1193 \\
\hline C 0.455418 & -1.985056 & -3.532672 & 829.1101 & 829.1989 & 835.2556 \\
\hline H 0.184960 & -2.560121 & -4.410020 & 836.9216 & 838.5340 & 839.5211 \\
\hline C - 0.139291 & -0.731683 & -3.313136 & 872.1738 & 872.3884 & 893.1125 \\
\hline C - 0.897013 & 0.155529 & -4.148372 & 897.0825 & 898.6520 & 902.5199 \\
\hline H -1.341866 & -0.105058 & -5.098536 & 917.0982 & 917.9417 & 921.1869 \\
\hline C - 0.867866 & 1.402007 & -3.570098 & 921.5964 & 923.1037 & 926.9012 \\
\hline
\end{tabular}




\begin{tabular}{|c|c|c|c|c|c|}
\hline H -1.286321 & 2.312874 & -3.964913 & 944.0156 & 945.4932 & 951.5941 \\
\hline C -0.088462 & 1.307115 & -2.361834 & 952.2170 & 982.7714 & 993.1799 \\
\hline C 0.553937 & 2.258763 & -1.514110 & 999.0546 & 1007.3387 & 1045.1551 \\
\hline C 0.210251 & 3.697250 & -1.517179 & 1065.7005 & 1066.2145 & 1077.1121 \\
\hline C - 1.319142 & 5.335281 & -2.220008 & 1081.4130 & 1083.7713 & 1085.4320 \\
\hline H -0.503444 & 5.996119 & -2.525557 & 1090.1586 & 1090.7289 & 1091.0347 \\
\hline H - 1.577603 & 5.566021 & -1.184210 & 1092.1696 & 1098.8707 & 1100.0372 \\
\hline C - 2.514226 & 5.444916 & -3.137228 & 1131.4485 & 1136.2572 & 1137.6064 \\
\hline H -2.243318 & 5.210088 & -4.170353 & 1140.5157 & 1144.7486 & 1157.6723 \\
\hline H -2.907235 & 6.465149 & -3.111379 & 1158.6995 & 1159.2965 & 1179.5848 \\
\hline H -3.308301 & 4.763518 & -2.821855 & 1196.9428 & 1197.7196 & 1198.6308 \\
\hline O 0.807214 & 4.547216 & -0.884661 & 1199.7246 & 1206.7803 & 1210.6753 \\
\hline O -0.860569 & 3.974590 & -2.271912 & 1210.8202 & 1217.6991 & 1223.1369 \\
\hline B - 0.720535 & -0.877549 & 0.988925 & 1231.8965 & 1233.5645 & 1249.2328 \\
\hline В 0.982729 & -0.595685 & -1.043760 & 1265.0055 & 1266.4518 & 1283.9268 \\
\hline N -0.171884 & -0.128069 & 2.190455 & 1284.3856 & 1314.6395 & 1318.8986 \\
\hline N -1.954571 & -0.083331 & 0.624139 & 1321.1495 & 1334.9718 & 1336.4802 \\
\hline N -1.294324 & -2.148499 & 1.583410 & 1338.4276 & 1341.0343 & 1354.0553 \\
\hline N 0.238415 & -0.000809 & -2.226245 & 1359.3852 & 1359.5093 & 1389.1432 \\
\hline N 1.935983 & 0.509184 & -0.647849 & 1389.7770 & 1400.0536 & 1400.4291 \\
\hline N 1.897021 & -1.629103 & -1.671330 & 1407.3006 & 1407.9512 & 1419.5253 \\
\hline O 0.193891 & -1.171335 & -0.034975 & 1422.6019 & 1423.1897 & 1423.5736 \\
\hline C - 4.513835 & -2.629089 & -0.302121 & 1438.7848 & 1440.3090 & 1446.0677 \\
\hline$C-5.733065$ & -4.617926 & -0.666711 & 1446.9576 & 1450.2704 & 1453.7742 \\
\hline H -5.408410 & -5.649367 & -0.815868 & 1456.3009 & 1458.5076 & 1464.9669 \\
\hline C - 6.921638 & -4.539488 & 0.269119 & 1465.7370 & 1498.2936 & 1499.6201 \\
\hline H -6.675072 & -4.956102 & 1.249524 & 1500.6440 & 1500.8764 & 1509.4006 \\
\hline H -7.758598 & -5.110008 & -0.144883 & 1515.3536 & 1518.5231 & 1520.1965 \\
\hline H -7.242300 & -3.503460 & 0.395147 & 1520.2070 & 1520.5152 & 1521.5202 \\
\hline O -5.358940 & -2.007974 & -0.913510 & 1527.2227 & 1528.1604 & 1530.1233 \\
\hline
\end{tabular}




\begin{tabular}{|c|c|c|c|c|c|}
\hline O -4.581991 & -3.960959 & -0.114788 & 1530.7698 & 1530.8327 & 1535.2975 \\
\hline H -5.963781 & -4.168370 & -1.634288 & 1535.5416 & 1539.4575 & 1546.3950 \\
\hline C 5.122840 & -1.216816 & 0.220667 & 1547.3172 & 1549.4164 & 1567.3692 \\
\hline H 8.162749 & -4.457208 & 0.663042 & 1568.6025 & 1570.8808 & 1570.9284 \\
\hline C 6.862702 & -2.754998 & 0.564490 & 1593.6844 & 1593.8197 & 1599.4721 \\
\hline H 7.595779 & -2.059713 & 0.146065 & 1604.5032 & 1801.0265 & 1803.3411 \\
\hline H 6.825069 & -2.579926 & 1.643108 & 1814.6865 & 1816.2408 & 3086.5339 \\
\hline C 7.191356 & -4.192561 & 0.236094 & 3086.6192 & 3087.1852 & 3087.3352 \\
\hline H 6.438616 & -4.869154 & 0.648673 & 3101.2385 & 3106.6395 & 3106.8329 \\
\hline H 7.240966 & -4.347988 & -0.845020 & 3119.4599 & 3144.4788 & 3155.1298 \\
\hline O 5.760291 & -0.396385 & 0.848035 & 3155.3926 & 3162.0558 & 3169.2131 \\
\hline O 5.573969 & -2.466271 & 0.005195 & 3169.2572 & 3169.4177 & 3172.8140 \\
\hline C - -3.330306 & 1.619301 & 0.144677 & 3176.4680 & 3179.7336 & 3179.8760 \\
\hline C - 3.956460 & 0.458324 & -0.224653 & 3191.8850 & 3257.9909 & 3258.1590 \\
\hline C 2.763499 & 2.520736 & -0.109436 & 3287.5696 & 3287.6543 & 3288.5906 \\
\hline C 3.698564 & 1.578988 & 0.229984 & 3288.6127 & 3327.6741 & 3327.7229 \\
\hline H 4.677341 & 1.742862 & 0.650586 & 3335.8557 & 3335.9926 & 3337.7007 \\
\hline H 2.841732 & 3.591477 & -0.015464 & 3337.8347 & 3342.5575 & 3342.6219 \\
\hline H -3.714454 & 2.624301 & 0.082673 & & & \\
\hline H -4.943528 & 0.345567 & -0.642629 & & & \\
\hline
\end{tabular}


Table S13. Atomic symbols, Cartesian coordinates (in $\AA$ ) and vibrational frequencies $\left(\mathrm{cm}^{-1}\right)$ of the structure 2 optimized at MPW1PW91/6-31g(d).

Energy $=-2678.35078$ Hartree

Nimag $=$ nil

\begin{tabular}{|c|c|c|c|c|c|}
\hline Atom & $y$ & $z$ & \multicolumn{3}{|c|}{ Vibrational Frequencies } \\
\hline C-2.019623 & 1.202994 & 0.820543 & 7.3292 & 15.9070 & 20.5916 \\
\hline C - 3.273706 & 1.601082 & 0.084036 & 27.5733 & 32.9994 & 34.5187 \\
\hline H -3.019022 & 2.229023 & -0.773359 & 40.5502 & 46.3188 & 51.6013 \\
\hline H-3.923921 & 2.204938 & 0.719870 & 55.1243 & 69.0996 & 70.5896 \\
\hline C - 3.914712 & 0.250628 & -0.331672 & 74.0987 & 75.1545 & 79.3042 \\
\hline H -4.928829 & 0.120611 & 0.049906 & 81.9161 & 83.3931 & 88.2142 \\
\hline H -3.984590 & 0.138050 & -1.416852 & 95.7791 & 98.3088 & 100.8868 \\
\hline C - 2.981271 & -0.789236 & 0.234917 & 107.2962 & 123.2472 & 127.0694 \\
\hline C -3.110330 & -2.168546 & 0.405362 & 129.1869 & 130.8155 & 132.5616 \\
\hline C-2.186991 & -2.856444 & 1.281049 & 150.8584 & 152.1011 & 157.0885 \\
\hline C - 2.218938 & -4.076084 & 2.032332 & 167.3760 & 178.9543 & 187.6986 \\
\hline H-2.967786 & -4.844834 & 1.936258 & 196.1515 & 206.9014 & 230.5150 \\
\hline C - 1.170894 & -4.040603 & 2.931886 & 233.1004 & 235.1795 & 240.8400 \\
\hline H -0.945325 & -4.781028 & 3.686776 & 255.6665 & 256.4059 & 260.7057 \\
\hline C -0.485354 & -2.801976 & 2.754002 & 265.0836 & 269.5788 & 274.4793 \\
\hline C 0.422847 & -2.068231 & 3.529147 & 278.7465 & 287.0094 & 312.6809 \\
\hline H 0.892267 & -2.535718 & 4.387032 & 315.1120 & 333.0859 & 356.9633 \\
\hline C 0.581871 & -0.687957 & 3.324084 & 360.9411 & 363.6216 & 367.4133 \\
\hline C 1.029913 & 0.380953 & 4.152471 & 375.7932 & 377.3845 & 386.2868 \\
\hline H 1.566703 & 0.270783 & 5.084542 & 391.6887 & 394.6554 & 413.3658 \\
\hline C 0.569763 & 1.564037 & 3.602317 & 415.1678 & 416.9372 & 423.6272 \\
\hline H 0.676831 & 2.555812 & 4.010299 & 427.5438 & 429.3596 & 429.7941 \\
\hline C -0.164711 & 1.233773 & 2.420139 & 439.1084 & 446.6989 & 456.4095 \\
\hline C - 1.109262 & 1.937600 & 1.581149 & 459.4810 & 481.8667 & 493.0138 \\
\hline C - 1.252273 & 3.400689 & 1.587225 & 495.3060 & 501.4862 & 502.8975 \\
\hline
\end{tabular}




\begin{tabular}{|c|c|c|c|c|c|}
\hline C -0.315678 & 5.454617 & 2.241980 & 508.5513 & 514.6593 & 520.4086 \\
\hline H -1.295373 & 5.794967 & 2.587524 & 577.0635 & 577.7368 & 598.9308 \\
\hline H - 0.190843 & 5.769063 & 1.203385 & 623.0187 & 648.4238 & 668.1872 \\
\hline C 0.802067 & 5.960374 & 3.123359 & 668.2736 & 695.9157 & 696.2782 \\
\hline H 0.669508 & 5.631574 & 4.157819 & 711.3976 & 712.5606 & 732.6666 \\
\hline H 0.814940 & 7.053899 & 3.112001 & 739.7271 & 744.1440 & 751.3004 \\
\hline H 1.772257 & 5.604578 & 2.767205 & 758.9867 & 760.2350 & 767.5860 \\
\hline O - -2.136582 & 4.010020 & 1.012742 & 772.0619 & 773.3174 & 777.1043 \\
\hline O -0.281421 & 4.018769 & 2.280684 & 780.1068 & 784.3107 & 784.9839 \\
\hline C 1.498588 & 1.870612 & -0.842064 & 793.5027 & 798.5658 & 799.4247 \\
\hline C 2.539683 & 2.704749 & -0.139112 & 799.9259 & 808.1334 & 816.8058 \\
\hline H 2.098188 & 3.190339 & 0.734962 & 820.4065 & 825.9274 & 828.0319 \\
\hline H 2.897608 & 3.509346 & -0.783992 & 830.3869 & 831.9791 & 832.3576 \\
\hline C 3.649486 & 1.687893 & 0.237702 & 834.9724 & 835.3313 & 837.1106 \\
\hline H 4.622282 & 1.936692 & -0.190032 & 882.7756 & 883.0639 & 883.2561 \\
\hline H 3.805932 & 1.618420 & 1.317336 & 886.6340 & 887.5678 & 889.6976 \\
\hline C 3.140985 & 0.373185 & -0.298044 & 904.0420 & 904.3001 & 913.7306 \\
\hline C 3.760115 & -0.864697 & -0.477603 & 914.0011 & 916.2069 & 920.7196 \\
\hline C 3.124256 & -1.850293 & -1.323628 & 924.1203 & 926.2484 & 979.3463 \\
\hline C 3.575419 & -2.981577 & -2.078692 & 986.7505 & 988.4788 & 992.1033 \\
\hline H 4.556065 & -3.422675 & -2.010000 & 1013.8325 & 1018.8374 & 1025.7776 \\
\hline C 2.558536 & -3.340418 & -2.942223 & 1026.4609 & 1032.2928 & 1043.5619 \\
\hline H 2.594763 & -4.119713 & -3.690786 & 1043.7143 & 1069.1268 & 1079.9944 \\
\hline C 1.473817 & -2.435912 & -2.738265 & 1083.0737 & 1083.9564 & 1084.5951 \\
\hline C 0.335346 & -2.092613 & -3.479525 & 1086.5281 & 1087.4740 & 1097.1935 \\
\hline H 0.041220 & -2.707477 & -4.322299 & 1099.9317 & 1106.6082 & 1134.4748 \\
\hline C -0.310309 & -0.863603 & -3.265293 & 1137.4125 & 1141.8212 & 1155.5523 \\
\hline C - 1.140984 & -0.039719 & -4.077670 & 1157.8916 & 1158.5076 & 1166.8805 \\
\hline H -1.628579 & -0.347299 & -4.992365 & 1174.5020 & 1184.2632 & 1184.8372 \\
\hline C - 1.125241 & 1.236003 & -3.541759 & 1197.2300 & 1197.3462 & 1198.2100 \\
\hline
\end{tabular}




\begin{tabular}{|c|c|c|c|c|c|}
\hline H-1.596248 & 2.116830 & -3.946436 & 1199.1544 & 1202.9260 & 1209.2739 \\
\hline C - 0.285700 & 1.208109 & -2.384064 & 1218.1884 & 1229.6637 & 1234.0306 \\
\hline C 0.361429 & 2.215961 & -1.573255 & 1235.7507 & 1246.2974 & 1246.7525 \\
\hline C - 0.037898 & 3.630843 & -1.581172 & 1256.6095 & 1262.1382 & 1264.7318 \\
\hline C - 1.662847 & 5.201967 & -2.227096 & 1280.0261 & 1282.0975 & 1293.1882 \\
\hline H -0.874973 & 5.873243 & -2.578628 & 1293.5510 & 1314.0590 & 1316.5825 \\
\hline H - 1.888237 & 5.452529 & -1.188098 & 1317.7968 & 1318.5205 & 1320.8189 \\
\hline C - 2.892147 & 5.265281 & -3.102772 & 1342.4055 & 1344.8408 & 1353.6921 \\
\hline H - 2.654563 & 5.002300 & -4.137281 & 1354.4865 & 1354.9991 & 1376.9791 \\
\hline H -3.299561 & 6.280184 & -3.093835 & 1378.3809 & 1412.8766 & 1414.4718 \\
\hline H -3.666452 & 4.584451 & -2.739907 & 1420.3794 & 1420.9668 & 1421.2798 \\
\hline O 0.570739 & 4.522429 & -1.017358 & 1422.5646 & 1425.2556 & 1425.9157 \\
\hline O -1.173910 & 3.851358 & -2.264260 & 1440.8111 & 1441.1048 & 1443.9837 \\
\hline B - 0.612844 & -0.886730 & 1.092091 & 1445.4436 & 1452.9242 & 1457.3896 \\
\hline В 0.945520 & -0.591677 & -1.075863 & 1460.8048 & 1461.7288 & 1471.9149 \\
\hline N-0.060016 & -0.100510 & 2.261359 & 1471.9880 & 1477.3758 & 1477.6833 \\
\hline N -1.898674 & -0.141688 & 0.726816 & 1480.9189 & 1483.4275 & 1484.2955 \\
\hline N -1.110226 & -2.173566 & 1.707146 & 1486.9270 & 1501.9799 & 1502.7482 \\
\hline N 0.105596 & -0.071908 & -2.222565 & 1518.3401 & 1520.2902 & 1520.4130 \\
\hline N 1.881189 & 0.575790 & -0.750846 & 1520.5085 & 1521.0052 & 1523.1022 \\
\hline N 1.859607 & -1.612029 & -1.711902 & 1523.6389 & 1530.4218 & 1530.5057 \\
\hline O 0.262755 & -1.129754 & 0.023949 & 1530.6339 & 1539.7266 & 1546.7468 \\
\hline C -4.284038 & -2.816276 & -0.196528 & 1547.4517 & 1550.1004 & 1556.2934 \\
\hline C - 5.442128 & -4.849306 & -0.515674 & 1559.1448 & 1566.1956 & 1566.5779 \\
\hline H -5.087122 & -5.877018 & -0.613392 & 1570.2048 & 1571.4286 & 1615.7447 \\
\hline C - 6.662840 & -4.767059 & 0.377660 & 1616.4683 & 1617.3890 & 1621.8943 \\
\hline H -6.436387 & -5.134970 & 1.382111 & 1794.5684 & 1797.0395 & 1806.0093 \\
\hline H -7.470261 & -5.377984 & -0.037509 & 1807.6921 & 3085.9188 & 3086.0615 \\
\hline H -7.015141 & -3.736075 & 0.449303 & 3086.6210 & 3086.9701 & 3099.9103 \\
\hline O -5.126241 & -2.240633 & -0.859506 & 3107.4267 & 3107.8771 & 3118.1350 \\
\hline
\end{tabular}




\begin{tabular}{lllllll}
\hline O -4.328626 & -4.140853 & 0.046714 & 3119.2759 & 3119.4593 & 3125.5471 \\
H -5.653117 & -4.444475 & -1.507287 & 3125.7358 & 3142.9665 & 3154.5672 \\
C 5.108328 & -1.032339 & 0.083603 & 3154.8525 & 3159.6101 & 3159.7654 \\
H 8.328623 & -4.104584 & 0.454398 & 3161.4722 & 3168.2131 & 3168.3862 \\
C 6.935707 & -2.474757 & 0.390180 & 3168.6577 & 3171.5050 & 3173.2639 \\
H 7.626664 & -1.736409 & -0.026367 & 3173.3115 & 3175.9816 & 3178.4346 \\
H 6.900061 & -2.314712 & 1.471286 & 3178.5281 & 3190.8785 & 3252.5816 \\
C 7.339224 & -3.888464 & 0.041566 & 3252.9364 & 3285.4350 & 3285.6538 \\
H 6.629198 & -4.609696 & 0.454312 & 3285.8490 & 3285.9621 & 3330.8529 \\
H 7.383744 & -4.028549 & -1.041856 & 3331.0938 & 3333.5793 & 3334.1554 \\
O 5.707764 & -0.181107 & 0.712438 & & & & \\
O 5.628861 & -2.251033 & -0.154255 & & & & \\
\hline
\end{tabular}




\section{References:}

S1. Zhang, X. F.; Huang, J.; Xi, Q.; Wang, Y. Aust. J. Chem. 2010, 63, 1471; Mathai, S.; Smith, T. A.; Ghiggino, K. P. Photochem. Photobiol. Sci. 2007, 6, 995.

S2. SAINT, version 6.45 /8/6/03 and version 8.34A, Bruker AXS, 2003, 2014.

S3. Sheldrick, G. M. SADABS and SADABS 2014/5, Program for Empirical Absorption Correction of Area Detector Data, University of Göttingen, Germany, 1997, 2014

S4. (a) SHELXL -Version 2014/7; Program for the Solution and Refinement of Crystal Structures, University of Göttingen, Germany, 1993-2014; (b) Sheldrick, G. M.; Acta Cryst. 2008, A64, 112.

S5 (a) Spek, A. L. PLATON, A Multipurpose Crystallographic Tool, Utrecht University, Utrecht, The Netherlands, 2002. (b) A. L. Spek, J. Appl. Cryst., 2003, 36, 7.

S6. Weigend, F.; Ahlrichs, R. Phys. Chem. Chem. Phys. 2005, 7, 3297.

S7. Gaussian 09, Revision E.01, M. J. Frisch, G. W. Trucks, H. B. Schlegel, G. E. Scuseria, M. A. Robb, J. R. Cheeseman, G. Scalmani, V. Barone, B. Mennucci, G. A. Petersson, H. Nakatsuji, M. Caricato, X. Li, H. P. Hratchian, A. F. Izmaylov, J. Bloino, G. Zheng, J. L. Sonnenberg, M. Hada, M. Ehara, K. Toyota, R. Fukuda, J. Hasegawa, M. Ishida, T. Nakajima, Y. Honda, O. Kitao, H. Nakai, T. Vreven, J. A. Montgomery, Jr., J. E. Peralta, F. Ogliaro, M. Bearpark, J. J. Heyd, E. Brothers, K. N. Kudin, V. N. Staroverov, R. Kobayashi, J. Normand, K. Raghavachari, A. Rendell, J. C. Burant, S. S. Iyengar, J. Tomasi, M. Cossi, N. Rega, J. M. Millam, M. Klene, J. E. Knox, J. B. Cross, V. Bakken, C. Adamo, J. Jaramillo, R. Gomperts, R. E. Stratmann, O. Yazyev, A. J. Austin, R. Cammi, C. Pomelli, J. W. Ochterski, R. L. Martin, K. Morokuma, V. G. Zakrzewski, G. A. Voth, P. Salvador, J. J. Dannenberg, S. Dapprich, A. D. Daniels, Ö. Farkas, J. B. Foresman, J. V. Ortiz, J. Cioslowski, and D. J. Fox, Gaussian, Inc., Wallingford CT, 2009.

S8. Y. Zhao and D. G. Truhlar. J. Chem. Phys. 2006, 125, 194101; F. Weigend and R. Ahlrichs. Phys. Chem. Chem. Phys. 2005, 7, 3297.

S9. J. Aragó and A. Troisi, Phys. Rev. Lett. 2015, 114, 026402. 